Supporting Information

\title{
Iron-Catalyzed Regio- and Stereoselective Hydrosilylation of 1,3-Enynes to Access 1,3-Dienylsilanes
}

\author{
Zhihao Guo, ${ }^{\dagger, \ddagger}$ Huanan Wen, ${ }^{\ddagger}$ Guixia Liu, ${ }^{*, \ddagger}$ Zheng Huang, ${ }^{*},, \neq, \S$ \\ ${ }^{\dagger}$ Chang-Kung Chuang Institute, East China Normal University, Shanghai 200062, \\ China \\ ${ }^{\ddagger}$ State Key Laboratory of Organometallic Chemistry, Center for Excellence in \\ Molecular Synthesis, Shanghai Institute of Organic Chemistry, Chinese Academy of \\ Sciences, 345 Lingling Road, Shanghai 200032, China \\ ${ }^{\S}$ School of Chemistry and Material Sciences, Hangzhou Institute for Advanced Study, \\ University of Chinese Academy of Sciences, 1 Sub-lane Xiangshan, Hangzhou \\ 310024, China
}

guixia@sioc.ac.cn

huangzh@sioc.ac.cn 


\section{Table of Contents}

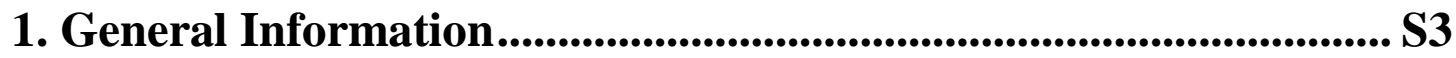

2. Synthesis of 1,3-Enynes ............................................................................. S4

3. Iron Catalyzed Hydrosilylation of 1,3-Enynes................................... S8

3.1 General Procedure:.............................................................................................

3.2 Chracterization of 1,3-Dienylsilanes: ..................................................................

4. Derivatization of 1,3-Dienylsilanes................................................... S16

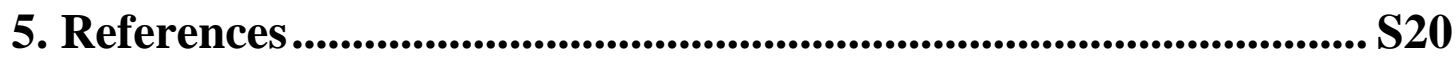

6. Copies of NMR Spectra......................................................................... S22 


\section{General Information}

Unless otherwise noted, all reagents were purchased from commercial suppliers and used without further purification. All manipulations were carried out using standard Schlenk, high-vacuum and glovebox techniques. Tetrahydrofuran (THF) was purchased from Sinopharm Group Chemical Co., Ltd. and dried over sodium overnight under an atmosphere of dry argon, then distilled from sodium benzophenone ketyl prior to use. Hexane was distilled from calcium hydride prior to use. Iron complexes $\mathbf{1} \mathbf{a}^{[1]}, \mathbf{1 b}^{[2]}, \mathbf{1} \mathbf{c}^{[3]}, \mathbf{1 d}^{[4]}, \mathbf{1 e}^{[4]}$ were prepared according to previously reported procedures.

NMR spectra were recorded on Agilent $400 \mathrm{MHz}$, Bruker $400 \mathrm{MHz}$ or Varian Mercury $400 \mathrm{MHz} .{ }^{1} \mathrm{H}$ NMR chemical shifts were referenced to residual solvent peak or tetramethylsilane signal (0 ppm), and ${ }^{13} \mathrm{C}$ NMR chemical shifts were referenced to the solvent resonances. Data for ${ }^{1} \mathrm{H}$ NMR are recorded as follows: chemical shift $(\delta$, ppm), multiplicity ( $\mathrm{s}=$ singlet, $\mathrm{d}=$ doublet, $\mathrm{t}=$ triplet, $\mathrm{q}=$ quartet, $\mathrm{m}=$ multiplet or unresolved, coupling constant (s) in $\mathrm{Hz}$, integration). Data for ${ }^{13} \mathrm{C}$ NMR are reported in terms of chemical shift ( $\delta, \mathrm{ppm})$. GC analysis was acquired on Agilent 7890A gas chromatograph equipped with a flame-ionization detector. GC-MS analysis was performed on Agilent 7890A gas chromatograph coupled to an Agilent 5975C inert mass selective detector. High resolution mass spectrometer (HR-MS) were carried out by the Analytical Laboratory of Shanghai Institute of Organic Chemistry (CAS). 


\section{Synthesis of 1,3-Enynes}

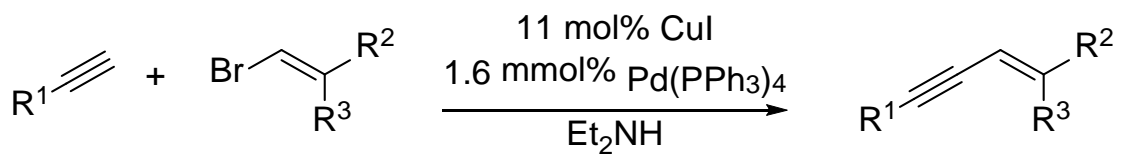

\section{General Procedure A:}

Under argon, terminal alkyne (1 equiv) and alkenyl bromide (1.3 equiv) was added to the solution of $\mathrm{Pd}\left(\mathrm{PPh}_{3}\right)_{4}$ (0.016 equiv) and $\mathrm{CuI}$ (0.11 equiv) in $\mathrm{Et}_{2} \mathrm{NH}(0.5 \mathrm{M})$. After stirring at $50{ }^{\circ} \mathrm{C}$ for $12 \mathrm{~h}$, the reaction was quenched by exposing to air. The reaction mixture was filtered, and the filtrate was evaporated under vacuum. The residue was purified by flash column chromatography to give the title compound.

General Procedure B: Similar to General Procedure A, expect that 1.3 equivalent of terminal alkyne and 1 equivalent of alkenyl bromide were used.

$\mathbf{2} \mathbf{a}^{[5]}, \mathbf{2} \mathbf{c}^{[6]}, \mathbf{2} \mathbf{d}^{[7]}, \mathbf{2} \mathbf{f}^{[7]}, \mathbf{2} \mathbf{g}^{[8]}, \mathbf{2} \mathbf{h}^{[9]}, \mathbf{2} \mathbf{m}^{[8]}, \mathbf{2} \mathbf{n}^{[8]}, \mathbf{4} \mathbf{a}^{[5]}, \mathbf{4} \mathbf{b}^{[10]}, \mathbf{4} \mathbf{c}^{[11]}$ were synthesized following the known procedure and data match those previously reported.

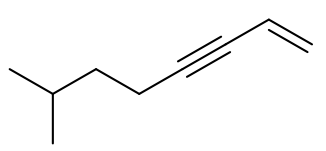

7-methyl-1-octene-3-yne (2e) was afforded as a light yellow-green liquid (732 mg, 60\%) following the General Procedure A after flash column chromatography eluting with PE (petroleum ether). ${ }^{1} \mathrm{H}$ NMR (400 $\mathrm{MHz} \mathrm{CDCl}_{3}$ ) $\delta 5.79$ (ddt, $J=17.5,11.0$, $2.2 \mathrm{~Hz}, 1 \mathrm{H}$ ), 5.55 (dd, $J=17.5,2.3 \mathrm{~Hz}, 1 \mathrm{H}$ ), 5.37 (dd, $J=11.0,2.3 \mathrm{~Hz}, 1 \mathrm{H}$ ), 2.32 (td, $J=7.4,2.2 \mathrm{~Hz}, 2 \mathrm{H}), 1.71$ (hept, $J=6.7 \mathrm{~Hz}, 1 \mathrm{H}$ ), 1.44 (q, $J=7.3 \mathrm{~Hz}, 2 \mathrm{H}), 0.92$ (d, $J=$ 6.7 Hz, 6H). ${ }^{13} \mathrm{C}$ NMR (101 MHz, $\left.\mathrm{CDCl}_{3}\right) \delta 125.3,117.7,91.2,79.2,37.6,27.2,22.1$, 17.3. HRMS-FI $(\mathrm{m} / \mathrm{z})$ : Calcd for $\mathrm{C}_{9} \mathrm{H}_{14}[\mathrm{M}]^{+}$122.1096; found: 122.1090 .<smiles>C=CC#CCCOCc1cccc(C(F)(F)F)c1</smiles>

6-(3-Trifluoromethylphenyl)methoxy-1-hexene-3-yne (2i) was afforded as a light yellow liquid (310 mg, 48\%) following the General Procedure A after flash column chromatography eluting with PE/EtOAc (v/v 30:1). ${ }^{1} \mathrm{H}$ NMR (400 MHz, $\left.\mathrm{CDCl}_{3}\right){ }^{1} \mathrm{H}$ NMR (400 MHz, Chloroform-d) $\delta 7.63$ (s, 1H), 7.57-7.52 (m, 2H), 7.49-7.43 (m, 1H), 5.85-5.72 (m, 1H), 5.58 (dd, $J=17.4,2.2 \mathrm{~Hz}, 1 \mathrm{H}$ ), 5.42 (dd, $J=11.0,2.2 \mathrm{~Hz}$, 
1H), 4.61 (s, 2H), 3.64 (t, $J=6.9 \mathrm{~Hz}, 2 \mathrm{H}), 2.65$ (td, $J=6.9,2.1 \mathrm{~Hz}, 2 \mathrm{H}) .{ }^{13} \mathrm{C} \mathrm{NMR}$ $\left(101 \mathrm{MHz}, \mathrm{CDCl}_{3}\right) \delta 139.3,130.8\left(\mathrm{q}, \mathrm{C}-\mathrm{F},{ }^{2} J_{\mathrm{C}-\mathrm{F}}=32.3 \mathrm{~Hz}\right), 130.7,128.9,126.2$, $124.4\left(\mathrm{q}, \mathrm{C}-\mathrm{F},{ }^{3} J_{\mathrm{C}-F}=3.8 \mathrm{~Hz}\right), 124.2\left(\mathrm{q}, \mathrm{C}-\mathrm{F},{ }^{1} J_{C-F}=273.4 \mathrm{~Hz}\right), 124.1\left(\mathrm{q}, \mathrm{C}-\mathrm{F},{ }^{3} J_{C-F}=\right.$ $3.9 \mathrm{~Hz}), 117.3,87.3,80.4,72.1,68.7,20.7 .{ }^{19} \mathrm{~F}$ NMR (377 $\left.\mathrm{MHz}, \mathrm{CDCl}_{3}\right) \delta-62.6$. HRMS-EI (m/z): Calcd for $\mathrm{C}_{14} \mathrm{H}_{13} \mathrm{~F}_{3} \mathrm{O}[\mathrm{M}]^{+}$254.0928; found:254.0909.

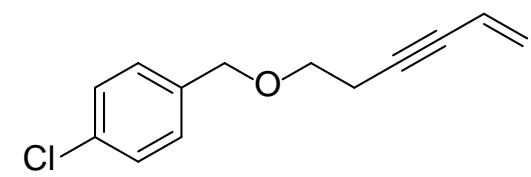

4-(4-chlorophenyl)methoxy-1-hexene-3-yne (2j) was afforded as a light yellow liquid (495 mg, 78\%) following the General Procedure A after flash column chromatography eluting with PE/EtOAc (v/v 30:1). ${ }^{1} \mathrm{H}$ NMR (400 MHz, $\left.\mathrm{CDCl}_{3}\right) \delta$ 7.32 (d, $J=8.5 \mathrm{~Hz}, 2 \mathrm{H}$ ), 7.28 (d, $J=8.6 \mathrm{~Hz}, 2 \mathrm{H}$ ), 5.78 (ddt, $J=17.5,11.0,2.1 \mathrm{~Hz}$, 1H), 5.58 (dd, $J=17.5,2.2 \mathrm{~Hz}, 1 \mathrm{H}$ ), 5.41 (dd, $J=11.0,2.2 \mathrm{~Hz}, 1 \mathrm{H}$ ), 4.52 (s, 2H), $3.59(\mathrm{t}, J=6.9 \mathrm{~Hz}, 2 \mathrm{H}), 2.62(\mathrm{td}, J=6.9,2.1 \mathrm{~Hz}, 2 \mathrm{H}) .{ }^{13} \mathrm{C} \mathrm{NMR}\left(101 \mathrm{MHz}, \mathrm{CDCl}_{3}\right) \delta$ 136.8, 133.5, 129.1, 128.7, 126.3, 117.5, 87.6, 80.5, 72.3, 68.6, 20.9. HRMS-EI (m/z): Calcd for $\mathrm{C}_{13} \mathrm{H}_{13} \mathrm{ClO}[\mathrm{M}]^{+}$220.0655; found: 220.0647.

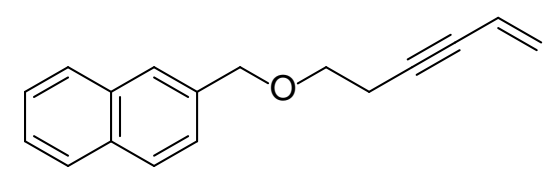

4-(2-naphthyl)methoxy-1-hexene-3-yne (2k) was afforded as a light yellow liquid (834 mg, 56\%) following the General Procedure A after flash column chromatography with PE/EtOAc (v/v 15:1). ${ }^{1} \mathrm{H}$ NMR (400 MHz, $\left.\mathrm{CDCl}_{3}\right) \delta 7.94-7.84$ (m, 4H), 7.59-7.53 (m, 3H), 5.94 (ddt, $J=17.6,11.0,2.2 \mathrm{~Hz}, 1 \mathrm{H}$ ), 5.73 (dd, $J=17.5$, $2.3 \mathrm{~Hz}, 1 \mathrm{H}$ ), 5.52 (dd, $J=11.0,2.3 \mathrm{~Hz}, 1 \mathrm{H}), 4.76$ (s, 2H), 3.71 (t, $J=7.0 \mathrm{~Hz}, 2 \mathrm{H}$ ), $2.76(\mathrm{td}, J=7.1,2.0 \mathrm{~Hz}, 2 \mathrm{H}) .{ }^{13} \mathrm{C}$ NMR $\left(101 \mathrm{MHz}, \mathrm{CDCl}_{3}\right) \delta 135.8,133.5,133.2$, 128.3, 128.0, 127.9, 126.5, 126.3, 126.0, 125.8, 117.7, 87.9, 80.6, 73.1, 68.5, 21.0. HRMS-EI (m/z): Calcd for $\mathrm{C}_{17} \mathrm{H}_{16} \mathrm{O}[\mathrm{M}]^{+} 236.1201$; found: 236.1193.<smiles>C=CC#CCNC(=O)c1ccccc1</smiles>

5-benzamide-1-pentene-3-yne (2l) was afforded as a light yellow liquid (760 mg, 
68\%) following the General Procedure A after flash column chromatography eluting with PE/EtOAc (v/v 2:1). ${ }^{1} \mathrm{H}$ NMR (400 MHz, $\left.\mathrm{CDCl}_{3}\right) \delta 7.79(\mathrm{dd}, 2 \mathrm{H}), 7.55-7.41$ (m, 3H), 6.34 (s, 1H), 5.80 (ddt, $J=17.6,10.9,2.0 \mathrm{~Hz}, 1 \mathrm{H}$ ), 5.66 (dd, $J=17.6,2.3 \mathrm{~Hz}$, 1H), 5.50 (dd, $J=10.9,2.3 \mathrm{~Hz}, 1 \mathrm{H}), 4.38$ (dd, $J=5.2,2.0 \mathrm{~Hz}, 2 \mathrm{H}) .{ }^{13} \mathrm{C}$ NMR $(101$ $\left.\mathrm{MHz}, \mathrm{CDCl}_{3}\right) \delta 167.2,133.8,131.7,128.6,127.6,127.1,116.6,85.5,82.2,30.5$. HRMS-EI (m/z): Calcd for $\mathrm{C}_{12} \mathrm{H}_{11} \mathrm{NO}[\mathrm{M}]^{+} 185.0841$; found: 185.0846.

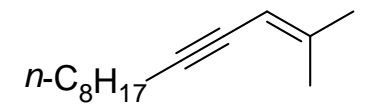

2-methyl-(E)-2-tridecen-4-yne (2o) was afforded as a light yellow liquid (1.65 g, 86\%) following the General Procedure B after flash column chromatography eluting with PE. ${ }^{1} \mathrm{H}$ NMR (400 MHz, $\left.\mathrm{CDCl}_{3}\right) \delta 5.23(\mathrm{~s}, 1 \mathrm{H}), 2.31$ (t, $\left.J=6.9 \mathrm{~Hz}, 2 \mathrm{H}\right), 1.86$ (s, 3H), 1.76 (s, 3H), 1.52 (p, $J=6.8 \mathrm{~Hz}, 2 \mathrm{H}), 1.45-1.34$ (m, 2H), 1.33-1.22 (m, 8H), $0.88(\mathrm{t}, J=6.7 \mathrm{~Hz}, 3 \mathrm{H}) .{ }^{13} \mathrm{C} \mathrm{NMR}\left(101 \mathrm{MHz}, \mathrm{CDCl}_{3}\right) \delta 146.5,105.6,92.2,78.5,31.9$, 29.3, 29.2, 29.1, 29.0, 24.6, 22.7, 20.7, 19.6, 14.1. HRMS-EI (m/z): Calcd for $\mathrm{C}_{14} \mathrm{H}_{24}$ $[\mathrm{M}]^{+}$192.1878; found: 192.1886 .

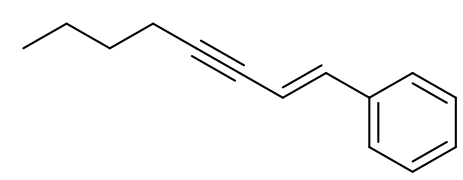

(E)-1-phenyl-1-octene-3-yne (2p) was afforded as a colorless liquid (417 mg, 85\%) following the General Procedure B after flash column chromatography eluting with PE/EtOAc (v/v 30:1). ${ }^{1} \mathrm{H}$ NMR (400 MHz, $\left.\mathrm{CDCl}_{3}\right) \delta 7.40-7.27$ (m, 5H), 6.87 (d, $J=$ 16.2 Hz, 1H), 6.16 (dt, $J=16.2,2.3 \mathrm{~Hz}, 1 \mathrm{H}), 2.38$ (td, $J=7.1,2.3 \mathrm{~Hz}, 2 \mathrm{H}), 1.59-1.52$ (m, 2H), 1.51-1.42 (m, 2H), 0.95 (t, $J=7.2 \mathrm{~Hz}, 3 \mathrm{H}) .{ }^{13} \mathrm{C}$ NMR (101 MHz, $\left.\mathrm{CDCl}_{3}\right) \delta$ 140.0, 136.6, 128.7, 128.2, 126.1, 108.9, 93.1, 79.7, 30.9, 22.0, 19.4, 13.7. These spectroscopic data correspond to reported data ${ }^{[12]}$.

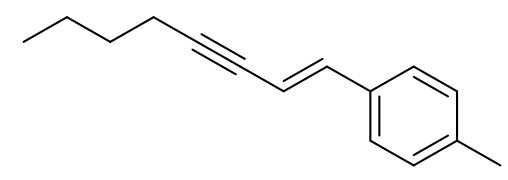

(E)-1-p-methylphenyl-1-octene-3-yne (2q) was afforded as a light yellow liquid (699 mg, 70\%) following the General Procedure B after flash column chromatography eluting with PE/EtOAc (v/v 30:1). ${ }^{1} \mathrm{H}$ NMR (400 $\left.\mathrm{MHz}, \mathrm{CDCl}_{3}\right) \delta$ 7.28 (d, $J=8.1 \mathrm{~Hz}, 2 \mathrm{H}$ ), 7.14 (d, $J=7.9 \mathrm{~Hz}, 2 \mathrm{H}), 6.87$ (d, $J=16.2 \mathrm{~Hz}, 1 \mathrm{H}), 6.14$ (dt, $J=16.2,2.2 \mathrm{~Hz}, 1 \mathrm{H}), 2.40$ (td, $J=7.0,1.9 \mathrm{~Hz}, 2 \mathrm{H}), 2.36$ (s, 3H), 1.61-1.45 (m, 4H), 
0.97 (t, $J=7.2 \mathrm{~Hz}, 3 \mathrm{H}) .{ }^{13} \mathrm{C}$ NMR $\left(101 \mathrm{MHz}, \mathrm{CDCl}_{3}\right) \delta 139.9,138.2,133.9,129.3$, 126.0, 107.8, 92.5, 79.9, 30.9, 22.0, 21.2, 19.3, 13.6. HRMS-EI $(\mathrm{m} / \mathrm{z})$ : Calcd for $\mathrm{C}_{15} \mathrm{H}_{18}[\mathrm{M}]^{+}$198.1409; found: 198.1404 .

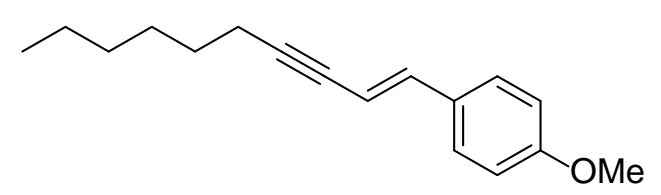

(E)-1-p-methoxyphenyl-1-decene-3-yne (2r) was afforded as a light yellow liquid (2.45 g, 92\%) following the General Procedure B after flash column chromatography eluting with PE/EtOAc (v/v 30:1). ${ }^{1} \mathrm{H}$ NMR (400 $\left.\mathrm{MHz}, \mathrm{CDCl}_{3}\right) \delta$ 7.30 (d, $J=8.7 \mathrm{~Hz}, 2 \mathrm{H}), 6.88-6.79$ (m, 3H), 6.02 (dt, $J=16.2,2.2 \mathrm{~Hz}, 1 \mathrm{H}$ ), 3.81 (s, 3H), 2.36 (td, $J=7.1,2.2 \mathrm{~Hz}, 2 \mathrm{H}), 1.61-1.52$ (m, 2H), 1.47-1.38 (m, 2H), 1.37-1.29 (m, 4H), 0.91 (t, $J=6.8 \mathrm{~Hz}, 3 \mathrm{H}) .{ }^{13} \mathrm{C} \mathrm{NMR}\left(101 \mathrm{MHz}, \mathrm{CDCl}_{3}\right) \delta 159.8,139.5,129.5$, 127.3, 114.1, 106.6, 92.2, 80.0, 55.3, 31.5, 28.9, 28.7, 22.6, 19.7, 14.1. HRMS-EI (m/z): Calcd for $\mathrm{C}_{17} \mathrm{H}_{22} \mathrm{O}[\mathrm{M}]^{+}$242.1671; found: 242.1666 .

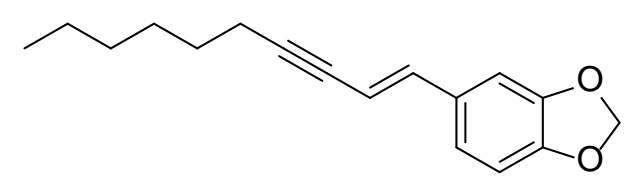

(E)-1-[3,4-(methylenedioxy)phenyl]-1-decene-3-yne (2s) was afforded as a light yellow liquid (535 mg, 61\%) following the General Procedure B after flash column chromatography eluting with PE/EtOAc (v/v 3:1). ${ }^{1} \mathrm{H}$ NMR $\left(400 \mathrm{MHz}, \mathrm{CDCl}_{3}\right) \delta 6.89$ (d, $J=1.6 \mathrm{~Hz}, 1 \mathrm{H}), 6.81-6.72(\mathrm{~m}, 3 \mathrm{H}), 5.99$ (dt, $J=16.2,2.3 \mathrm{~Hz}, 1 \mathrm{H}), 5.94$ (s, 2H), 2.36 (td, $J=7.1,2.3 \mathrm{~Hz}, 2 \mathrm{H}), 1.56$ (p, $J=7.1 \mathrm{~Hz}, 2 \mathrm{H}), 1.47-1.38$ (m, 2H), 1.36-1.27 (m, 4H), 0.91 (t, $J=6.9 \mathrm{~Hz}, 3 \mathrm{H}) .{ }^{13} \mathrm{C}$ NMR $\left(101 \mathrm{MHz}, \mathrm{CDCl}_{3}\right) \delta 148.1,147.8,139.6$, 131.1, 121.2, 108.3, 107.0, 105.0, 101.2, 92.6, 79.8, 31.4, 28.8, 28.7, 22.6, 19.7, 14.1. HRMS-EI (m/z): Calcd for $\mathrm{C}_{17} \mathrm{H}_{20} \mathrm{O}_{2}[\mathrm{M}]^{+}$256.1463; found: 256.1475. 


\section{Iron Catalyzed Hydrosilylation of 1,3-Enynes}

\subsection{General Procedure:}
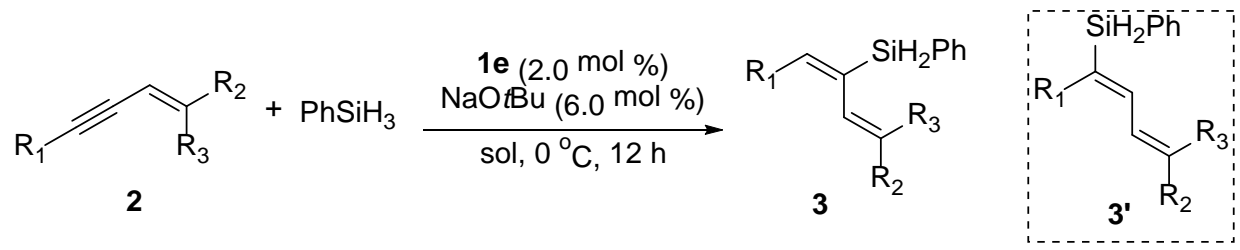

Under argon, $\mathrm{NaOtBu}(18 \mu \mathrm{L}, 0.5 \mathrm{M}$ in THF, $0.018 \mathrm{mmol}, 0.06$ equiv) was added to a solution of $\mathrm{PhSiH}_{3}$ (35.7 mg, $0.33 \mathrm{mmol}, 1.1$ equiv) and 1 e $(5.3 \mathrm{mg}, 0.006 \mathrm{mmol}$, 0.02 equiv) in hexane ( $2 \mathrm{~mL})$. After the resulting mixture was cooled to $-20{ }^{\circ} \mathrm{C}$, 1,3-enynes ( $0.3 \mathrm{mmol}, 1$ equiv) was added. The reaction was stirred at $0{ }^{\circ} \mathrm{C}$ for $12 \mathrm{~h}$. After that, the reaction was quenched by exposing to air, then the mixture was filtered, and the filtrate was evaporated under vacuum. The residue was purified by chromatography to give the title compound. The ratio of 3:3' (rr) was determined by ${ }^{1} \mathrm{H}$ NMR or GC.

\subsection{Chracterization of 1,3-Dienylsilanes:}

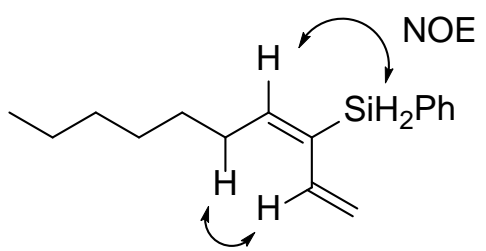

(E)-3-phenylsilyl-1,3-decadiene (3a), colorless viscous liquid (62.2 mg, 85\%), $\mathrm{R}_{f}=$ 0.9 (PE), $r r$ (3a:3a') 94:6. ${ }^{1} \mathrm{H}$ NMR (400 MHz, $\left.\mathrm{CDCl}_{3}\right) \delta 7.58(\mathrm{dd}, J=6.0,1.7 \mathrm{~Hz}$, 2H), 7.43-7.32 (m, 3H), 6.87 (dd, $J=17.5,10.7 \mathrm{~Hz}, 1 \mathrm{H}), 6.14$ (t, $J=7.2 \mathrm{~Hz}, 1 \mathrm{H}$ ), 5.26 (d, $J=17.5 \mathrm{~Hz}, 1 \mathrm{H}), 5.15$ (d, $J=10.7 \mathrm{~Hz}, 1 \mathrm{H}$ ), 4.67 (s, 2H), 2.30 (q, $J=7.3 \mathrm{~Hz}$, 2H), 1.45-1.37 (m, 2H), 1.36-1.26 (m, 6H), 0.89 (t, $J=6.2 \mathrm{~Hz}, 3 \mathrm{H}) .{ }^{13} \mathrm{C}$ NMR (101 $\left.\mathrm{MHz}, \mathrm{CDCl}_{3}\right) \delta 149.5,135.5,134.9,132.1,131.6,129.6,128.0,117.6,31.7,29.2$, 29.0, 22.6, 14.1. HRMS-EI (m/z): Calcd for $\mathrm{C}_{16} \mathrm{H}_{24} \mathrm{Si}[\mathrm{M}]^{+}$244.1647; found: 244.1644. The configuration of the silyl substituted double bond was determined by the NOESY spectrum (see page S32). 


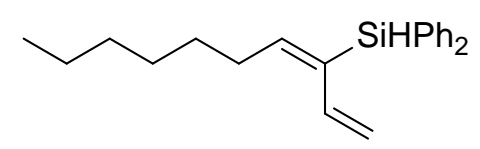

(E)-3- diphenylsilyl -1,3-decadiene (3b), colorless viscous liquid (82.1 mg, 85\%), $\mathrm{R}_{f}$ $=0.7$ (PE), $r r$ (3b:3b') 98:2. ${ }^{1} \mathrm{H}$ NMR (400 MHz, $\left.\mathrm{CDCl}_{3}\right) \delta$ 7.67-7.57 (m, 4H), 7.50-7.35 (m, 6H), 6.96 (ddd, $J=17.7,10.9,1.3 \mathrm{~Hz}, 1 \mathrm{H}$ ), 6.04 (t, $J=7.2 \mathrm{~Hz}, 1 \mathrm{H}$ ), 5.34 (s, 1H), 5.30 (d, $J=17.7 \mathrm{~Hz}, 1 \mathrm{H}), 5.17$ (dd, $J=10.9,1.5 \mathrm{~Hz}, 1 \mathrm{H}$ ), 2.37 (q, $J=$ $7.3 \mathrm{~Hz}, 2 \mathrm{H}), 1.49-1.41$ (m, 2H), $1.41-1.27(\mathrm{~m}, 6 \mathrm{H}), 0.94(\mathrm{t}, J=6.3 \mathrm{~Hz}, 3 \mathrm{H}) .{ }^{13} \mathrm{C}$ NMR (101 MHz, $\left.\mathrm{CDCl}_{3}\right) \delta 149.74,135.80,135.02,133.71,132.87,129.57,127.95$, 117.72, 31.68, 29.21, 29.19, 29.01, 22.64, 14.10. HRMS-EI (m/z): Calcd for $\mathrm{C}_{22} \mathrm{H}_{28} \mathrm{Si}$ $[\mathrm{M}]^{+}$320.1960; found: 320.1958 .<smiles>C=C/C(=C\CCCCCCCC)[SH+]c1ccccc1</smiles>

(E)-3-phenylsilyl-1,3-dodecadiene (3c) colorless viscous liquid (64.7 mg, 79\%), $\mathrm{R}_{f}=$ 0.9 (PE), $r r$ (3c:3c') 94:6. ${ }^{1} \mathrm{H}$ NMR (400 MHz, $\left.\mathrm{CDCl}_{3}\right) \delta$ 7.60-7.56 (m, 2H), 7.42-7.32 (m, 3H), 6.86 (dd, $J=17.5,10.7 \mathrm{~Hz}, 1 \mathrm{H}), 6.14$ (t, $J=7.2 \mathrm{~Hz}, 1 \mathrm{H}), 5.26$ (d, $J=17.5 \mathrm{~Hz}, 1 \mathrm{H}), 5.15$ (d, $J=10.6 \mathrm{~Hz}, 1 \mathrm{H}), 4.66$ (s, 2H), 2.30 (q, $J=7.3 \mathrm{~Hz}, 2 \mathrm{H})$, 1.46-1.37 (m, 2H), 1.34-1.23 (m, 10H), 0.89 (t, $J=6.8 \mathrm{~Hz}, 3 \mathrm{H}) .{ }^{13} \mathrm{C}$ NMR (101 MHz, $\left.\mathrm{CDCl}_{3}\right) \delta 149.5,135.4,134.9,132.0,131.6,129.6,128.0,117.5,31.9,29.4,29.3,29.3$, 29.2, 29.1, 22.7, 14.1. HRMS-EI (m/z): Calcd for $\mathrm{C}_{18} \mathrm{H}_{28} \mathrm{Si}[\mathrm{M}]^{+}$272.1960; found: 272.1958.<smiles>C=C/C(=C\CCc1ccccc1)[SH+]c1ccccc1</smiles>

(E)-3-phenylsilyl-6-phenyl-1,3-hexadiene (3d) colorless viscous liquid (53.6 $\mathrm{mg}$, 68\%), $\mathrm{R}_{f}=0.8$ (PE), rr (3d:3d') 94:6. ${ }^{1} \mathrm{H}$ NMR (400 MHz, $\mathrm{CDCl}_{3}$ ) $\delta$ 7.62-7.54 (m, 2H), 7.45-7.36 (m, 3H), 7.36-7.29 (m, 2H), 7.25-7.19 (m, 3H), 6.85 (dd, $J=17.5$, $10.7 \mathrm{~Hz}, 1 \mathrm{H}$ ), 6.20 (t, $J=7.0 \mathrm{~Hz}, 1 \mathrm{H}), 5.30$ (d, $J=17.5 \mathrm{~Hz}, 1 \mathrm{H}), 5.18$ (d, $J=10.7 \mathrm{~Hz}$, 1H), 4.70 (s, 2H), 2.81-2.74 (m, 2H), 2.71-2.63 (m, 2H). ${ }^{13} \mathrm{C}$ NMR (101 MHz, $\left.\mathrm{CDCl}_{3}\right) \delta 147.8,141.6,135.6,134.8,132.7,131.9,129.8,128.6,128.6,128.2,126.1$, 118.2, 35.5, 31.1. HRMS-EI $(\mathrm{m} / \mathrm{z})$ : Calcd for $\mathrm{C}_{18} \mathrm{H}_{20} \mathrm{Si}[\mathrm{M}]^{+}$264.1334; found: 264.1333. 
$\overbrace{}^{\mathrm{SiH}_{2} \mathrm{Ph}}$

(E)-3-phenylsilyl-7-methyl-1,3-octadiene (3e), colorless viscous liquid (38.5 mg, 56\%), $\mathrm{R}_{f}=0.8(\mathrm{PE}), \operatorname{rr}\left(3 \mathbf{e}: 3 \mathrm{e}^{\prime}\right)$ 95:5. ${ }^{1} \mathrm{H}$ NMR (400 MHz, $\mathrm{CDCl}_{3}$ ) $\delta 7.58$ (dd, $J=7.7$, $1.6 \mathrm{~Hz}, 2 \mathrm{H}), 7.42-7.32$ (m, 3H), 6.87 (dd, $J=17.6,10.7 \mathrm{~Hz}, 1 \mathrm{H}), 6.13$ (t, $J=7.2 \mathrm{~Hz}$, 1H), 5.26 (d, $J=17.5 \mathrm{~Hz}, 1 \mathrm{H}$ ), 5.15 (d, $J=10.7 \mathrm{~Hz}, 1 \mathrm{H}$ ), 4.66 (s, 2H), 2.31 (q, $J=7.5$ $\mathrm{Hz}, 2 \mathrm{H}), 1.64-1.52$ (m, 1H), 1.31 (q, 2H), 0.90 (d, $J=6.6 \mathrm{~Hz}, 6 \mathrm{H}) .{ }^{13} \mathrm{C}$ NMR (101 $\left.\mathrm{MHz}, \mathrm{CDCl}_{3}\right) \delta 149.6,135.5,134.8,132.0,131.5,129.6,128.0,117.6,38.3,27.8$, 27.1, 22.5. HRMS-EI (m/z): Calcd for $\mathrm{C}_{15} \mathrm{H}_{22} \mathrm{Si}[\mathrm{M}]^{+}$230.1491; found: 230.1497.<smiles>C=C/C(=C\CC1CCCCC1)[SnH]c1ccccc1</smiles>

(E)-3-phenylsilyl-5-cyclohexyl-1,3-pentadiene (3f), colorless viscous liquid (65.0 mg, 84\%), $\mathrm{R}_{f}=0.8$ (PE), $r r$ (3f:3f') 98:2. ${ }^{1} \mathrm{H}$ NMR (400 MHz, $\left.\mathrm{CDCl}_{3}\right) \delta 7.61$ (dd, $J=$ 7.7, $1.8 \mathrm{~Hz}, 2 \mathrm{H}$ ), 7.47-7.34 (m, 3H), 6.89 (ddd, $J=17.5,10.7,1.2 \mathrm{~Hz}, 1 \mathrm{H}), 6.20$ (t, $J$ $=7.4 \mathrm{~Hz}, 1 \mathrm{H}$ ), 5.28 (d, $J=17.6 \mathrm{~Hz}, 1 \mathrm{H}), 5.16$ (d, $J=10.7 \mathrm{~Hz}, 1 \mathrm{H}), 4.71(\mathrm{~s}, 2 \mathrm{H}), 2.23$ $(\mathrm{t}, J=7.1 \mathrm{~Hz}, 2 \mathrm{H}), 1.81-1.63(\mathrm{~m}, 4 \mathrm{H}), 1.49-1.36(\mathrm{~m}, 1 \mathrm{H}), 1.32-1.09(\mathrm{~m}, 4 \mathrm{H})$, 1.03-0.88 (m, 2H). ${ }^{13} \mathrm{C}$ NMR (101 MHz, $\left.\mathrm{CDCl}_{3}\right) \delta 148.2,135.5,135.1,132.4,132.1$, 129.6, 128.0, 117.5, 38.2, 36.9, 33.3, 26.5, 26.4. HRMS-EI (m/z): Calcd for $\mathrm{C}_{17} \mathrm{H}_{24} \mathrm{Si}$ $[\mathrm{M}]^{+}$256.1647; found: 256.1652 .<smiles>C=C/C(=C\CCO[AsH3])[SH2]c1ccccc1</smiles>

(E)-3-phenylsilyl-6-tert-butyldimethylsiloxy-1,3-hexadiene (3g), light yellow viscous liquid (56.4 mg, 59\%), $\mathrm{R}_{f}=0.8$ (30:1 PE/EtOAc), $r r$ (3g:3g') 96:4. ${ }^{1} \mathrm{H}$ NMR $\left(400 \mathrm{MHz}, \mathrm{CDCl}_{3}\right) \delta 7.60(\mathrm{~d}, J=6.7 \mathrm{~Hz}, 2 \mathrm{H}), 7.42-7.33$ (m, 3H), 6.87 (dd, $J=17.6$, $10.7 \mathrm{~Hz}, 1 \mathrm{H}$ ), 6.16 (t, $J=7.1 \mathrm{~Hz}, 1 \mathrm{H}), 5.30$ (d, $J=17.5 \mathrm{~Hz}, 1 \mathrm{H}), 5.18$ (d, $J=10.7 \mathrm{~Hz}$, 1H), 4.68 (s, 2H), 3.70 (t, $J=6.7 \mathrm{~Hz}, 2 \mathrm{H}), 2.55$ (q, $J=6.8 \mathrm{~Hz}, 2 \mathrm{H}), 0.90$ (s, 9H), 0.05 (s, 6H). ${ }^{13} \mathrm{C}$ NMR (101 MHz, $\left.\mathrm{CDCl}_{3}\right) \delta 145.0,135.5,134.9,133.6,131.8,129.6$, 128.0, 117.9, 62.2, 32.8, 25.9, 18.3, -5.3. HRMS-EI (m/z): Calcd for $\mathrm{C}_{14} \mathrm{H}_{21} \mathrm{OSi}_{2}$ [M-C $\left.{ }_{4} \mathrm{H}_{9}\right]^{+}$261.1131; found: 261.1142. 
TBDPS

(E)-3-phenylsilyl-6-tert-butyldiphenylsiloxy-1,3-hexadiene (3h), red viscous liquid (118.0 mg, 89\%), $\mathrm{R}_{f}=0.9$ (10:1 PE/EtOAc), rr (3h:3h') 93:7. ${ }^{1} \mathrm{H}$ NMR (400 MHz, $\left.\mathrm{CDCl}_{3}\right) \delta 7.75-7.70(\mathrm{~m}, 3 \mathrm{H}), 7.66-7.62(\mathrm{~m}, 2 \mathrm{H}), 7.52-7.35$ (m, 10H), 6.85 (ddd, $J=$ 17.5, 10.7, 1.2 Hz, 1H), 6.26 (t, $J=7.2 \mathrm{~Hz}, 1 \mathrm{H}$ ), 5.34 (d, $J=17.5 \mathrm{~Hz}, 1 \mathrm{H}), 5.21$ (d, $J$ $=10.6 \mathrm{~Hz}, 1 \mathrm{H}), 4.75$ (s, 2H), 3.80 (t, $J=6.5 \mathrm{~Hz}, 2 \mathrm{H}), 2.63$ (q, $J=6.7 \mathrm{~Hz}, 2 \mathrm{H}), 1.11$ (s, 9H). ${ }^{13} \mathrm{C}$ NMR (101 MHz, $\left.\mathrm{CDCl}_{3}\right) \delta 145.1,145.1,135.7,135.6,135.0,133.8,133.8$, 131.8, 129.7, 128.1, 127.7, 118.0, 63.0, 32.6, 26.9, 19.2. HRMS-EI $(\mathrm{m} / \mathrm{z})$ : Calcd for $\mathrm{C}_{24} \mathrm{H}_{25} \mathrm{OSi}_{2}\left[\mathrm{M}-\mathrm{C}_{4} \mathrm{H}_{9}\right]^{+}$385.1444; found: 385.1439.<smiles>C=C/C(=C\CCOCc1cccc(C(F)(F)F)c1)[SH+]c1ccccc1</smiles>

(E)-3-phenylsilyl-6-(3-trifluoromethylbenzyl)oxy-1,3-hexadiene (3i), colorless viscous liquid (69.9 mg, 64\%), $\mathrm{R}_{f}=0.7$ (20:1 PE/EtOAc), $r r$ (3i:3i’) 97:3. ${ }^{1} \mathrm{H}$ NMR (400 MHz, $\mathrm{CDCl}_{3}$ ) $\delta$ 7.64-7.42 (m, 6H), 7.43-7.32 (m, 3H), 6.88 (ddd, $J=17.5,10.7$, $1.2 \mathrm{~Hz}, 1 \mathrm{H}), 6.19$ (t, $J=7.1 \mathrm{~Hz}, 1 \mathrm{H}), 5.33$ (d, $J=17.6 \mathrm{~Hz}, 1 \mathrm{H}), 5.22$ (d, $J=10.7 \mathrm{~Hz}$, $1 \mathrm{H}), 4.70$ (s, 2H), 4.57 (s, 2H), 3.60 (t, $J=6.8 \mathrm{~Hz}, 2 \mathrm{H}), 2.68$ (q, $J=6.8 \mathrm{~Hz}, 2 \mathrm{H}) .{ }^{13} \mathrm{C}$ NMR $\left(101 \mathrm{MHz}, \mathrm{CDCl}_{3}\right) \delta 144.2,139.5,135.5,134.7,134.2,131.7,103.8$ (q, $\left.\mathrm{C}-\mathrm{F},{ }^{2} J_{\mathrm{C}-\mathrm{F}}=32.2 \mathrm{~Hz}\right), 130.7,129.8,128.9,128.1,124.4\left(\mathrm{q}, \mathrm{C}-\mathrm{F},{ }^{3} J_{C-F}=3.8 \mathrm{~Hz}\right)$, 124.24 (q, C-F, $\left.{ }^{1} J_{C-F}=273.7 \mathrm{~Hz}\right), 124.15$ (q, C-F, $\left.{ }^{3} J_{C-F}=3.7 \mathrm{~Hz}\right), 118.5,72.2,69.6$, 29.8. ${ }^{19} \mathrm{~F}$ NMR (376 MHz, $\left.\mathrm{CDCl}_{3}\right) \delta$-62.6. HRMS-FI ( $\mathrm{m} / \mathrm{z}$ ): Calcd for $\mathrm{C}_{20} \mathrm{H}_{21} \mathrm{~F}_{3} \mathrm{OSi}$ $[\mathrm{M}]^{+}$362.1314; found: 362.1315 .<smiles>C=C/C(=C\CCOCc1ccc(Cl)cc1)[SH+]c1ccccc1</smiles>

(E)-3-phenylsilyl-6-(4-chlorobenzyl)oxy-1,3-hexadiene (3j), colorless viscous liquid (42.0 mg, 43\%), $\mathrm{R}_{f}=0.6$ (30:1 PE/EtOAc), $r r$ (3j:3j') 95:5. ${ }^{1} \mathrm{H}$ NMR (400 MHz, $\left.\mathrm{CDCl}_{3}\right) \delta 7.62-7.56(\mathrm{~m}, 2 \mathrm{H}), 7.43-7.34(\mathrm{~m}, 3 \mathrm{H}), 7.31$ (d, $\left.J=8.4 \mathrm{~Hz}, 2 \mathrm{H}\right), 7.25$ (d, $J=$ $8.0 \mathrm{~Hz}, 2 \mathrm{H}), 6.86$ (dd, $J=17.5,10.7 \mathrm{~Hz}, 1 \mathrm{H}), 6.16$ (t, $J=7.0 \mathrm{~Hz}, 1 \mathrm{H}), 5.32$ (d, $J=$ $17.6 \mathrm{~Hz}, 1 \mathrm{H}), 5.21$ (d, $J=10.6 \mathrm{~Hz}, 1 \mathrm{H}), 4.69$ (s, 2H), 4.48 (s, 2H), 3.55 (t, $J=6.7 \mathrm{~Hz}$, 2H), 2.65 (q, $J=6.8 \mathrm{~Hz}, 2 \mathrm{H}) .{ }^{13} \mathrm{C}$ NMR $\left(101 \mathrm{MHz}, \mathrm{CDCl}_{3}\right) \delta 144.3,136.9,135.5$, 134.7, 134.0, 133.3, 131.7, 129.7, 128.9, 128.6, 128.1, 118.4, 72.2, 69.3, 29.8. 
HRMS-FI (m/z): Calcd for $\mathrm{C}_{19} \mathrm{H}_{21} \mathrm{OClSi}[\mathrm{M}]^{+}$328.1050; found: 328.1047.<smiles>C=C/C(=C\CCOCc1ccc2ccccc2c1)[SH-]c1ccccc1</smiles>

(E)-3-phenylsilyl-6-(2-naphthylmethyl)oxy-1,3-hexadiene (3k), light yellow viscous liquid (60.4 mg, 58\%), $\mathrm{R}_{f}=0.8$ (30:1 PE/EtOA), $r r$ (3k:3k’) 94:6. ${ }^{1} \mathrm{H}$ NMR $\left(400 \mathrm{MHz}, \mathrm{CDCl}_{3}\right) \delta$ 7.87-7.79 (m, 4H), 7.65-7.59 (m, 2H), 7.53-7.47 (m, 3H), 7.43-7.34 (m, 3H), 6.91 (dd, $J=17.5,10.7 \mathrm{~Hz}, 1 \mathrm{H}$ ), 6.23 (t, $J=7.1 \mathrm{~Hz}, 1 \mathrm{H}$ ), 5.34 (d, $J=17.5 \mathrm{~Hz}, 1 \mathrm{H}$ ), 5.22 (d, $J=10.8 \mathrm{~Hz}, 1 \mathrm{H}), 4.73$ (s, 2H), 4.71 (s, 2H), 3.63 (t, $J=6.8$ $\mathrm{Hz}, 2 \mathrm{H}), 2.70$ (q, $J=6.9 \mathrm{~Hz}, 2 \mathrm{H}) .{ }^{13} \mathrm{C}$ NMR $\left(101 \mathrm{MHz}, \mathrm{CDCl}_{3}\right) \delta 144.5,135.8,135.5$, 134.7, 133.9, 133.3, 133.0, 131.7, 129.7, 128.2, 128.0, 127.9, 127.7, 126.4, 126.1, 125.9, 125.7, 118.3, 73.0, 69.2, 29.8. HRMS-EI $(\mathrm{m} / \mathrm{z})$ : Calcd for $\mathrm{C}_{23} \mathrm{H}_{24} \mathrm{OSi}[\mathrm{M}]^{+}$ 344.1596; found: 344.1592 .<smiles>C=C/C(=C\CNC(=O)c1ccccc1)Sc1ccccc1</smiles>

(E)-3-phenylsilyl-5-benzamide-1,3-pentadiene (3I), light yellow viscous liquid (26.1 mg, 30\%) using THF as the solvent, $\mathrm{R}_{f}=0.5$ (4:1 PE/EtOAc), $r r$ (3l:3l') 97:3. ${ }^{1} \mathrm{H}$ NMR (400 MHz, Chloroform-d) $\delta$ 7.79-7.75 (m, 2H), 7.58 (dd, $J=7.8,1.6$ Hz, 2H), 7.53-7.47 (m, 1H), 7.45-7.34 (m, 5H), 6.87 (ddd, $J=17.4,10.7,1.2 \mathrm{~Hz}$, 1H), 6.33 (br, 1H), 6.13 (t, $J=6.5 \mathrm{~Hz}, 1 \mathrm{H}$ ), 5.37 (d, $J=17.4 \mathrm{~Hz}, 1 \mathrm{H}), 5.28$ (d, $J=$ $10.6 \mathrm{~Hz}, 1 \mathrm{H}), 4.68$ (s, 2H), 4.34 (t, $J=6.0 \mathrm{~Hz}, 2 \mathrm{H}) .{ }^{13} \mathrm{C}$ NMR (101 MHz, $\left.\mathrm{CDCl}_{3}\right) \delta$ 167.4, 142.3, 135.6, 135.6, 134.3, 134.0, 131.6, 131.0, 129.9, 128.6, 128.2, 127.0, 119.9, 38.3. HRMS-EI $(\mathrm{m} / \mathrm{z})$ : Calcd for $\mathrm{C}_{18} \mathrm{H}_{19} \mathrm{NOSi}[\mathrm{M}]^{+}$293.1236; found: 293.1234.<smiles>C=C/C(=C\CCOC(=O)c1ccccc1)[SH+]c1ccccc1</smiles>

(E)-3-phenylsilyl-1,3-pentadiene-6-benzoate (3m), light yellow viscous liquid (38.3 mg, 41\%) using THF as the solvent, $\mathrm{R}_{f}=0.7$ (10:1 PE/EtOAc), $r r\left(3 \mathrm{~m}: 3 \mathrm{~m}^{\prime}\right)$ 95:5. ${ }^{1} \mathrm{H}$ 
NMR (400 MHz, Chloroform- $d$ ) $\delta 8.02$ (dd, $J=8.4,1.4$ Hz, 2H), 7.61-7.53 (m, 3H), 7.48-7.28 (m, 5H), 6.90 (ddd, $J=17.5,10.7,1.2 \mathrm{~Hz}, 1 \mathrm{H}$ ), 6.19 (t, $J=7.3 \mathrm{~Hz}, 1 \mathrm{H}$ ), 5.34 (d, $J=17.5 \mathrm{~Hz}, 1 \mathrm{H}$ ), 5.23 (d, $J=10.7 \mathrm{~Hz}, 1 \mathrm{H}), 4.70$ (s, 2H), 4.40 (t, $J=6.7 \mathrm{~Hz}$, 2H), 2.80 (q, $J=6.8 \mathrm{~Hz}, 2 \mathrm{H}) .{ }^{13} \mathrm{C}$ NMR $\left(101 \mathrm{MHz}, \mathrm{CDCl}_{3}\right) \delta 166.6,142.8,135.5$, 135.1, 134.5, 133.0, 131.4, 130.2, 129.8, 129.6, 128.4, 128.1, 118.9, 63.7, 28.6. HRMS-EI (m/z): Calcd for $\mathrm{C}_{19} \mathrm{H}_{20} \mathrm{O}_{2} \mathrm{Si}[\mathrm{M}]^{+}$308.1233; found: 308.1224 .<smiles>C=C/C(=C\CCCCCl)Sc1ccccc1</smiles>

(E)-3-phenylsilyl-8-chloro-1,3-octadiene (3n), colorless viscous liquid (52.6 mg, 70\%), $\mathrm{R}_{f}=0.8$ (PE), $r r$ (3n:3n') 96:4. ${ }^{1} \mathrm{H}$ NMR (400 MHz, $\left.\mathrm{CDCl}_{3}\right) \delta 7.65-7.59(\mathrm{~m}$, 2H), 7.45-7.37 (m, 3H), 6.88 (dd, $J=17.5,10.7 \mathrm{~Hz}, 1 \mathrm{H}), 6.15$ (t, $J=7.2 \mathrm{~Hz}, 1 \mathrm{H}$ ), 5.33 (d, $J=17.5 \mathrm{~Hz}, 1 \mathrm{H}$ ), 5.22 (d, $J=10.7 \mathrm{~Hz}, 1 \mathrm{H}$ ), 4.71 (s, 2H), 3.58 (t, $J=6.6 \mathrm{~Hz}$, 2H), 2.38 (q, $J=7.4 \mathrm{~Hz}, 2 \mathrm{H}), 1.89-1.79$ (m, 2H), 1.68-1.58 (m, 2H). ${ }^{13} \mathrm{C}$ NMR (101 $\left.\mathrm{MHz}, \mathrm{CDCl}_{3}\right) \delta 148.1,135.6,134.7,132.7,131.9,129.8,128.2,118.3,45.0,32.2$, 28.4, 26.5. HRMS-EI (m/z): Calcd for $\mathrm{C}_{14} \mathrm{H}_{19} \mathrm{ClSi}[\mathrm{M}]^{+}$250.0945; found: 250.0940 .

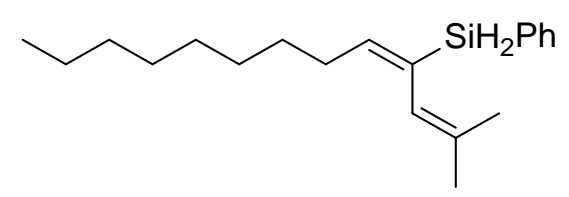

(E)-2-methyl-4-phenylsilyl-2,4-tridecadiene (3o), colorless viscous liquid (39.0 mg, 43\%), $\mathrm{R}_{f}=0.9$ (PE), $r r$ (3o:3o’) 99:1. ${ }^{1} \mathrm{H}$ NMR (400 MHz, $\left.\mathrm{CDCl}_{3}\right) \delta 7.58$ (dd, $J=7.7$, $1.7 \mathrm{~Hz}, 2 \mathrm{H}), 7.41-7.33$ (m, 3H), 6.04 (td, $J=6.9,1.6 \mathrm{~Hz}, 1 \mathrm{H}), 5.70$ (s, 1H), 4.56 (s, 2H), 2.07 (q, $J=7.1 \mathrm{~Hz}, 2 \mathrm{H}), 1.76$ (s, 3H), 1.53 (s, 3H), 1.44-1.33 (m, 3H), 1.31-1.26 (m, 9H), 0.90 (t, $J=6.8 \mathrm{~Hz}, 3 \mathrm{H}) .{ }^{13} \mathrm{C}$ NMR (101 MHz, $\left.\mathrm{CDCl}_{3}\right) \delta$ 147.28, 135.51, 133.72, 132.60, 132.38, 129.46, 127.88, 122.96, 31.93, 30.61, 29.50, 29.43, 29.31, 28.85, 25.54, 22.72, 19.49, 14.16. HRMS-EI $(\mathrm{m} / \mathrm{z})$ : Calcd for $\mathrm{C}_{20} \mathrm{H}_{32} \mathrm{Si}[\mathrm{M}]^{+}$ 300.2273; found: 300.2280 .<smiles>CCCC/C=C(/[SH2+])c1ccccc1</smiles>

(E)- 3-phenylsilyl-1-phenyl-1,3-octadiene (3p), light yellow viscous liquid (60.4 mg, 
69\%), $\mathrm{R}_{f}=0.7$ (PE), rr (3p:3p') 95:5. ${ }^{1} \mathrm{H}$ NMR (400 MHz, $\left.\mathrm{CDCl}_{3}\right) \delta$ 7.69-7.66 (m, 2H), 7.45-7.38 (m, 5H), 7.36-7.31 (m, 3H), 7.25-7.22 (m, 1H), 6.69 (d, J = $16.2 \mathrm{~Hz}$, 1H), 6.24 (t, $J=7.2 \mathrm{~Hz}, 1 \mathrm{H}), 4.82$ (s, 2H), 2.47 (q, $J=7.2 \mathrm{~Hz}, 2 \mathrm{H}), 1.54-1.39$ (m, 4H), $0.98(\mathrm{t}, J=7.1 \mathrm{~Hz}, 3 \mathrm{H}) .{ }^{13} \mathrm{C}$ NMR (101 MHz, $\left.\mathrm{CDCl}_{3}\right) \delta 149.6,137.8,135.5$, 132.3, 132.0, 131.0, 129.7, 128.6, 128.1, 127.5, 127.4, 126.3, 31.5, 29.1, 22.5, 14.0. HRMS-EI (m/z): Calcd for $\mathrm{C}_{20} \mathrm{H}_{24} \mathrm{Si}[\mathrm{M}]^{+}$292.1647; found: 292.1645 .<smiles>CCCC/C=C(/C=C/c1ccc(C)cc1)Sc1ccccc1</smiles>

(E)-3-phenylsilyl-1-p-methylphenyl-1,3-octadiene (3q), light yellow viscous liquid (71.7 mg, 78\%), $\mathrm{R}_{f}=0.7$ (PE), rr (3q:3q') 94:6. ${ }^{1} \mathrm{H}$ NMR (400 $\left.\mathrm{MHz}, \mathrm{CDCl}_{3}\right) \delta 7.65$ (dd, $J=7.6,1.8 \mathrm{~Hz}, 2 \mathrm{H}$ ), 7.43-7.37 (m, 3H), 7.34-7.27 (m, 3H), 7.13 (d, $J=7.9 \mathrm{~Hz}$, 2H), 6.65 (d, $J=16.2 \mathrm{~Hz}, 1 \mathrm{H}), 6.19$ (t, $J=7.2 \mathrm{~Hz}, 1 \mathrm{H}), 4.80$ (s, 2H), 2.44 (q, $J=7.2$ $\mathrm{Hz}, 2 \mathrm{H}), 2.35$ (s, 3H), 1.52-1.37 (m, 4H), 0.97 (t, $J=7.2 \mathrm{~Hz}, 3 \mathrm{H}) .{ }^{13} \mathrm{C}$ NMR (101 $\left.\mathrm{MHz}_{\mathrm{CDCl}}\right) \delta 149.1,137.3,135.5,135.0,132.3,132.1,131.0,129.6,129.3,128.0$, 126.5, 126.2, 31.5, 29.0, 22.5, 21.2, 14.0. HRMS-EI (m/z): Calcd for $\mathrm{C}_{21} \mathrm{H}_{26} \mathrm{Si}[\mathrm{M}]^{+}$ 306.1804; found: 306.1800 .

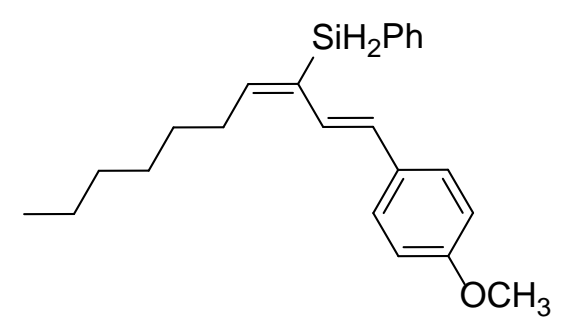

(E)-3-phenylsilyl-1-p-methoxyphenyl-1,3-decadiene (3r), light yellow viscous liquid (95.9 mg, 91\%), $\mathrm{R}_{f}=0.8$ (50:1 PE/EtOAc), rr (3r:3r') 95:5. ${ }^{1} \mathrm{H}$ NMR (400 $\left.\mathrm{MHz}, \mathrm{CDCl}_{3}\right) \delta 7.63(\mathrm{dd}, J=7.6,1.8 \mathrm{~Hz}, 2 \mathrm{H}), 7.40-7.35$ (m, 3H), 7.31 (d, $J=8.7 \mathrm{~Hz}$, 2H), 7.20 (dd, $J=16.2,1.2 \mathrm{~Hz}, 1 \mathrm{H}$ ), 6.84 (d, $J=8.8 \mathrm{~Hz}, 2 \mathrm{H}), 6.59$ (d, $J=16.2 \mathrm{~Hz}$, $1 \mathrm{H}$ ), 6.14 (t, $J=7.1 \mathrm{~Hz}, 1 \mathrm{H}), 4.76$ (s, 2H), 3.80 (s, 3H), 2.40 (q, $J=7.3 \mathrm{~Hz}, 2 \mathrm{H}$ ), 1.49-1.43 (m, 2H), 1.36-1.28 (m, 6H), 0.90 (t, $J=6.8 \mathrm{~Hz}, 3 \mathrm{H}) .{ }^{13} \mathrm{C}$ NMR (101 MHz, $\left.\mathrm{CDCl}_{3}\right) \delta 159.2,148.7,135.5,132.1,131.8,131.0,130.6,129.6,128.1,127.5,125.5$, 114.0, 55.3, 31.7, 29.3, 29.1, 22.7, 14.1. HRMS-EI (m/z): Calcd for $\mathrm{C}_{23} \mathrm{H}_{30} \mathrm{OSi}[\mathrm{M}]^{+}$ 350.2066; found: 350.2061 . 


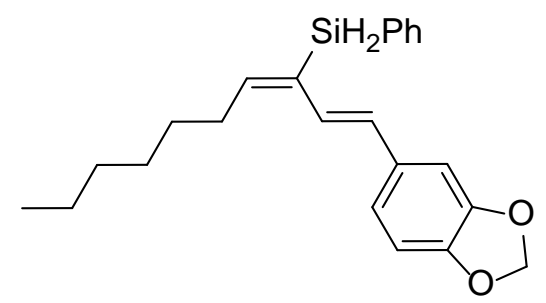

(E)-3-phenylsilyl-1-(3,4-methylenedioxyphenyl)phenyl-1,3-octadiene (3s), light yellow viscous liquid (91.0 mg, 83\%), $\mathrm{R}_{f}=0.7$ (50:1 PE/EtOAc), $r r$ (3s:3s’) 95:5. ${ }^{1} \mathrm{H}$ NMR (400 MHz, $\left.\mathrm{CDCl}_{3}\right) \delta 7.65$ (dd, $\left.J=7.6,1.8 \mathrm{~Hz}, 2 \mathrm{H}\right), 7.44-7.35$ (m, 3H), 7.19 (dd, $J=16.1,1.2 \mathrm{~Hz}, 1 \mathrm{H}), 6.97$ (d, $J=1.7 \mathrm{~Hz}, 1 \mathrm{H}), 6.83-6.73$ (m, 2H), 6.59 (d, $J=$ $16.1 \mathrm{~Hz}, 1 \mathrm{H}$ ), 6.18 (t, $J=7.2 \mathrm{~Hz}, 1 \mathrm{H}), 5.95$ (s, 2H), 4.79 (s, 2H), 2.43 (q, $J=7.3 \mathrm{~Hz}$, 2H), 1.55-1.45 (m, 2H), 1.43-1.29 (m, 6H), 0.93 (t, $J=6.8 \mathrm{~Hz}, 3 \mathrm{H}) .{ }^{13} \mathrm{C}$ NMR (101 $\left.\mathrm{MHz}, \mathrm{CDCl}_{3}\right) \delta 149.1,148.1,147.2,135.5,132.3,132.1,132.0,130.9,129.7,128.1$, 125.9, 121.3, 108.4, 105.4, 101.1, 31.8, 29.4, 29.3, 29.1, 22.7, 14.2. HRMS-EI (m/z): Calcd for $\mathrm{C}_{23} \mathrm{H}_{28} \mathrm{O}_{2} \mathrm{Si}[\mathrm{M}]^{+}$364.1859; found: 364.1853. 


\section{Derivatization of 1,3-Dienylsilanes}

\subsection{Hiyama-Denmark Coupling ${ }^{[13]}$}

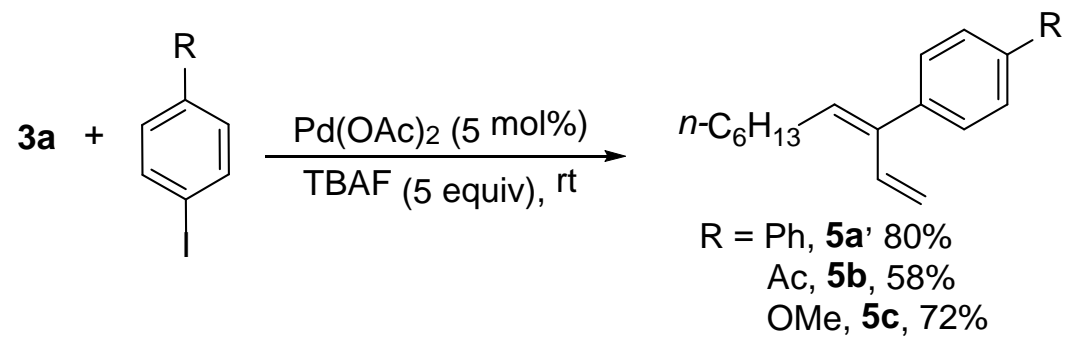

Under $\mathrm{N}_{2}$, a $10 \mathrm{~mL}$ Schlenk tube was charged with 3a (73.3 mg, $0.3 \mathrm{mmol}, 1.5$ equiv). TBAF ( $1 \mathrm{~mL}, 1 \mathrm{M}$ in THF, $1 \mathrm{mmol}, 5$ equiv) was added slowly and the mixture was stirred for 5 minutes. Iodobenzene (0.2 mmol, 1 equiv) and $\mathrm{Pd}(\mathrm{OAc})_{2}$ (4.5 mg, 0.02 mmol, 0.1 equiv) were then added. The reaction mixture was stirred at room temperature for $18 \mathrm{~h}$ and the resulting mixture was washed with aq. sat. $\mathrm{NH}_{4} \mathrm{Cl}$ and brine. The aqueous phase was extracted with EA $(3 \times 10 \mathrm{~mL})$. The combined organic layers were dried over $\mathrm{Na}_{2} \mathrm{SO}_{4}$ and evaporated under vacuum. The residue was purified by chromatography to give the title compound.<smiles>C=C/C(=C\CCCCCC)c1ccc(-c2ccccc2)cc1</smiles>

(E)-3-(p-phenylphenyl)-1,3-decadiene (5a), colorless viscous liquid (46.7 mg, 80\%), purified by chromatography eluting with PE/EtOAc (v/v 100:1). ${ }^{1} \mathrm{H}$ NMR (400 MHz, $\left.\mathrm{CDCl}_{3}\right) \delta 7.62(\mathrm{dd}, J=22.4,7.7 \mathrm{~Hz}, 4 \mathrm{H}), 7.47(\mathrm{t}, J=7.6 \mathrm{~Hz}, 2 \mathrm{H}), 7.42-7.34(\mathrm{~m}, 3 \mathrm{H})$, 6.92 (dd, $J=17.4,10.9 \mathrm{~Hz}, 1 \mathrm{H}), 5.67$ (t, $J=7.6 \mathrm{~Hz}, 1 \mathrm{H}$ ), 5.30 (d, $J=10.9 \mathrm{~Hz}, 1 \mathrm{H}$ ), 5.18 (d, $J=17.4 \mathrm{~Hz}, 1 \mathrm{H}), 2.36$ (q, $J=7.5 \mathrm{~Hz}, 2 \mathrm{H}), 1.57-1.47$ (m, 2H), 1.45-1.29 (m, $6 \mathrm{H}), 0.94(\mathrm{t}, J=6.7 \mathrm{~Hz}, 3 \mathrm{H}) .{ }^{13} \mathrm{C} \mathrm{NMR}\left(101 \mathrm{MHz}, \mathrm{CDCl}_{3}\right) \delta 141.1,140.9,139.8$, 139.4, 133.6, 133.3, 129.4, 128.9, 127.3, 127.2, 126.8, 117.4, 31.9, 29.8, 29.2, 28.4, 22.8, 14.3. HRMS-FI (m/z): Calcd for $\mathrm{C}_{22} \mathrm{H}_{26}[\mathrm{M}]^{+}$290.2035; found: 290.2027. 
<smiles>C=C/C(=C\CCCCCC)c1ccc(C(C)=O)cc1</smiles>

(E)-3-(p-acetylphenyl)-1,3-decadiene (5b), yellow viscous liquid (29.9 mg, 58\%), purified by chromatography eluting with PE/EtOAc (v/v 20:1). ${ }^{1} \mathrm{H}$ NMR (400 MHz, $\left.\mathrm{CDCl}_{3}\right) \delta 7.91(\mathrm{~d}, J=8.4 \mathrm{~Hz}, 2 \mathrm{H}), 7.36$ (d, $\left.J=8.4 \mathrm{~Hz}, 2 \mathrm{H}\right), 6.84$ (dd, $J=17.5,10.9$ Hz, 1H), 5.62 (t, $J=7.6 \mathrm{~Hz}, 1 \mathrm{H}), 5.26$ (d, $J=10.9 \mathrm{~Hz}, 1 \mathrm{H}), 5.03$ (d, $J=17.3 \mathrm{~Hz}, 1 \mathrm{H}$ ), 2.60 (s, 3H), 2.32 (q, $J=7.4$ Hz, 2H), 1.51-1.42 (m, 2H), 1.40-1.25 (m, 6H), 0.89 (t, $J=4.2 \mathrm{~Hz}, 3 \mathrm{H}) .{ }^{13} \mathrm{C}$ NMR $\left(101 \mathrm{MHz}, \mathrm{CDCl}_{3}\right) \delta 198.0,146.9,139.0,135.7,134.6$, 132.8, 129.1, 128.3, 117.7, 31.8, 29.6, 29.1, 28.3, 26.7, 22.7, 14.2. HRMS-EI (m/z): Calcd for $\mathrm{C}_{18} \mathrm{H}_{24} \mathrm{O}[\mathrm{M}]^{+}$256.1827; found: 256.1823 .<smiles>C=C/C(=C\CCCCCC)c1ccc(OC)cc1</smiles>

(E)-3-(p-phenylphenyl)-1,3-decadiene (5c), yellow viscous liquid (35.2 mg, 72\%), purified by chromatography eluting with PE/EtOAc (v/v 20:1). ${ }^{1} \mathrm{H}$ NMR (400 MHz, $\left.\mathrm{CDCl}_{3}\right) \delta 7.25-7.21(\mathrm{~m}, 2 \mathrm{H}), 6.93-6.84(\mathrm{~m}, 3 \mathrm{H}), 5.56(\mathrm{t}, J=7.5 \mathrm{~Hz}, 1 \mathrm{H}), 5.25(\mathrm{dt}, J$ = 10.9, $1.8 \mathrm{~Hz}, 1 \mathrm{H}$ ), 5.11 (dd, $J=17.4,1.9 \mathrm{~Hz}, 1 \mathrm{H}$ ), 3.85 (s, 3H), 2.32 (q, $J=7.4 \mathrm{~Hz}$, 2H), 1.53-1.44 (m, 2H), 1.41-1.28 (m, 6H), 0.93 (t, $J=6.8 \mathrm{~Hz}, 3 \mathrm{H}) .{ }^{13} \mathrm{C}$ NMR (101 $\left.\mathrm{MHz}, \mathrm{CDCl}_{3}\right) \delta 158.5,139.1,134.2,133.5,132.8,129.9,116.9,113.4,55.3,31.8$, 29.8, 29.1, 28.2, 22.7, 14.1. HRMS-EI $(\mathrm{m} / \mathrm{z})$ : Calcd for $\mathrm{C}_{17} \mathrm{H}_{24} \mathrm{O}[\mathrm{M}]^{+}$244.1827; found: 244.1821. The configuration of the double bond was determined by the NOESY spectrum (see page S55).

\subsection{Catalytic Hydrosilylation of Phenylacetylene ${ }^{[14]}$}

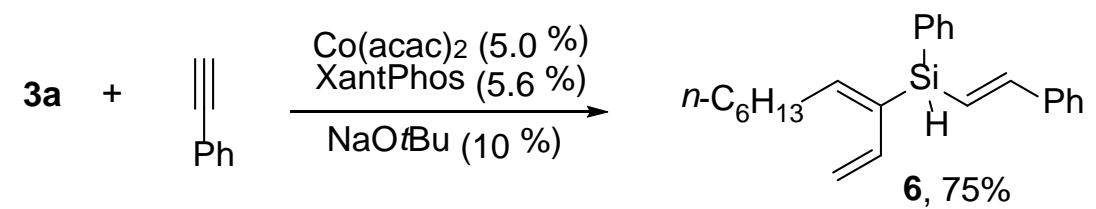

Under $\mathrm{N}_{2}$, Co(acac) $)_{2}$ (8.9 mg, 0.025 mmol, 0.05 equiv), XantPhos (16.2 mg, 0.028 mmol, 0.056 equiv) and THF ( $2 \mathrm{~mL}$ ) were added into a $10 \mathrm{~mL}$ Schlenk tube. The mixture was stirred at rt for 20 minutes, then 3a (122.2 mg, $0.5 \mathrm{mmol}, 1$ equiv) and 
phenylacetylene (66.4 mg, $0.65 \mathrm{mmol}, 1.3$ equiv) were introduced. After the resulting mixture was cooled to $-30{ }^{\circ} \mathrm{C}, \mathrm{NaOtBu}(100 \mu \mathrm{L}, 0.5 \mathrm{M}$ in THF, $0.05 \mathrm{mmol}, 0.1$ equiv) was added slowly. After stirring at room temperature for $12 \mathrm{~h}$, the reaction was quenched by adding EtOAc. The mixture was filtered, and the filtrate was evaporated under vacuum. The residue was purified by chromatography on silica gel with PE to afford 6 as a yellow viscous liquid (130 mg, 75\%). ${ }^{1} \mathrm{H}$ NMR (400 $\left.\mathrm{MHz}, \mathrm{CDCl}_{3}\right) \delta$ 7.71-7.65 (m, 2H), 7.57-7.50 (m, 2H), 7.49-7.31 (m, 6H), 7.14 (d, $J=19.0 \mathrm{~Hz}, 1 \mathrm{H}$ ), 6.95 (ddd, $J=17.6,10.7,1.3 \mathrm{~Hz}, 1 \mathrm{H}$ ), 6.71 (ddd, $J=19.0,3.3,1.5 \mathrm{~Hz}, 1 \mathrm{H}$ ), 6.17 (t, $J$ = 7.3 Hz, 1H), 5.37 (d, $J=17.7 \mathrm{~Hz}, 1 \mathrm{H}), 5.21$ (dd, $J=10.8,1.6 \mathrm{~Hz}, 1 \mathrm{H}), 5.10$ (s, $1 \mathrm{H}$ ), 2.39 (q, $J=7.4 \mathrm{~Hz}, 2 \mathrm{H}), 1.52-1.45$ (m, 2H), 1.40-1.33 (m, 6H), 0.96 (t, $J=6.1 \mathrm{~Hz}$, 3H). ${ }^{13} \mathrm{C}$ NMR $\left(101 \mathrm{MHz}, \mathrm{CDCl}_{3}\right) \delta 149.0,148.7,138.1,135.5,135.0,134.1,133.3$, 129.6, 128.6, 128.5, 128.0, 126.8, 122.1, 117.6, 31.7, 29.3, 29.3, 29.1, 22.7, 14.2. HRMS-EI (m/z): Calcd for $\mathrm{C}_{24} \mathrm{H}_{30} \mathrm{Si}[\mathrm{M}]^{+} 346.2117$; found: 346.2114 .

\subsection{Catalytic hydrosilylation of benzaldehyde ${ }^{[14]}$}

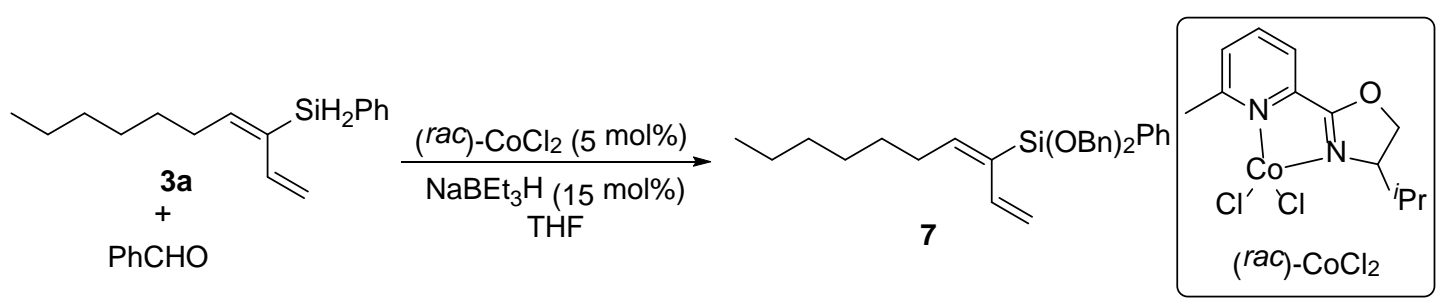

Under $\mathrm{N}_{2}$, (rac)- $\mathrm{CoCl}_{2}$ (8.4 mg, $0.025 \mathrm{mmol}, 0.05$ equiv) and THF (2 mL) were added to a $10 \mathrm{~mL}$ Schlenk tube and the mixture was stirred at rt for 5 minutes. After $\mathrm{NaBEt}_{3} \mathrm{H}$ (75 $\mu \mathrm{L}, 1 \mathrm{M}$ in THF, $0.075 \mathrm{mmol}, 0.15$ equiv) was added slowly at $-30{ }^{\circ} \mathrm{C}$, the resulting mixture was stirred at rt for 5 minutes and then $3 a$ (122.2 mg, $0.5 \mathrm{mmol}$, 1 equiv) and PhCHO (265.0 mg, 2.5 mmol, 5 equiv) were added. After stirring at room temperature for $12 \mathrm{~h}$, the reaction was quenched by air. The reaction mixture was filtered, and the filtrate was evaporated under vacuum. The residue was purified by chromatography on silica gel with PE/EtOAc (v/v 20:1), 7 was given as colorless viscous liquid (176 mg, 77\%). ${ }^{1} \mathrm{H}$ NMR (400 MHz, $\left.\mathrm{CDCl}_{3}\right) \delta 7.76-7.71$ (m, 2H), 7.41 (ddd, $J=14.0,7.8,6.1 \mathrm{~Hz}, 4 \mathrm{H}), 7.32$ (d, $J=4.5 \mathrm{~Hz}, 6 \mathrm{H}), 7.28-7.26(\mathrm{~m}, 1 \mathrm{H}$ ), 7.26-7.23 (m, 1H), 6.79 (ddd, $J=17.7,11.1,1.0 \mathrm{~Hz}, 1 \mathrm{H}$ ), 6.42 (t, $J=7.2 \mathrm{~Hz}, 1 \mathrm{H}$ ), 5.46 (d, $J=17.4 \mathrm{~Hz}, 1 \mathrm{H}$ ), 5.10 (d, $J=11.1 \mathrm{~Hz}, 2 \mathrm{H}$ ), 4.80 (s, 4H), 2.33 (q, $J=7.3 \mathrm{~Hz}$, 2H), 1.43 (q, $J=6.8 \mathrm{~Hz}, 2 \mathrm{H}), 1.37-1.28(\mathrm{~m}, 6 \mathrm{H}), 0.90(\mathrm{t}, J=6.5 \mathrm{~Hz}, 3 \mathrm{H}) .{ }^{13} \mathrm{C} \mathrm{NMR}$ $\left(101 \mathrm{MHz}, \mathrm{CDCl}_{3}\right) \delta 149.8,140.6,134.8,134.1,133.3,131.8,130.3,128.3,127.9$, 
127.1, 126.6, 117.8, 64.9, 31.8, 29.2, 29.1, 29.0, 22.7, 14.2. HRMS-EI (m/z): Calcd for $\mathrm{C}_{30} \mathrm{H}_{36} \mathrm{O}_{2} \mathrm{Si}[\mathrm{M}]^{+}$456.2485; found: 456.2495.

\subsection{Fluoride-Mediated Desilylation ${ }^{[15]}$}

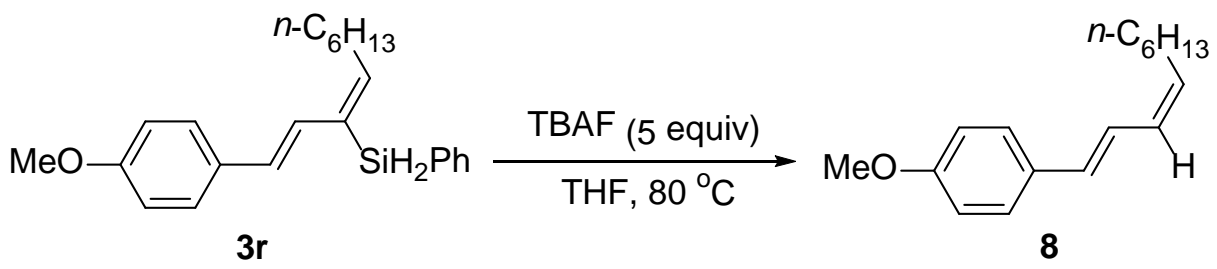

Under Ar, TBAF (1 M in THF, $0.4 \mathrm{~mL}, 0.4 \mathrm{mmol}$ ) was slowly added to a solution of 3r (70.1 mg, $0.2 \mathrm{mmol}$ ) in THF (2 mL). The reaction mixture was stirred at $80{ }^{\circ} \mathrm{C}$ for $12 \mathrm{~h}$, and then evaporated under vacuum. The residue was purified by chromatography on silica gel eluting with PE/EtOAc (v/v 30:1) to give 8 as colorless viscous liquid (41.9 mg, 86\%). ${ }^{1} \mathrm{H}$ NMR (400 $\left.\mathrm{MHz} \mathrm{CDCl}_{3}\right) \delta 7.36$ (d, $J=8.6 \mathrm{~Hz}$, 2H), 6.95 (dd, $J=15.5,11.1 \mathrm{~Hz}, 1 \mathrm{H}$ ), 6.87 (d, $J=8.7 \mathrm{~Hz}, 2 \mathrm{H}), 6.48$ (d, $J=15.6 \mathrm{~Hz}$, 1H), 6.14 (t, $J=10.9 \mathrm{~Hz}, 1 \mathrm{H}$ ), 5.49 (dt, $J=10.8,7.7 \mathrm{~Hz}, 1 \mathrm{H}$ ), 3.82 (s, 3H), 2.28 (q, $J$ $=7.3 \mathrm{~Hz}, 2 \mathrm{H}), 1.48-1.26(\mathrm{~m}, 8 \mathrm{H}), 0.90(\mathrm{t}, J=6.5 \mathrm{~Hz}, 3 \mathrm{H}) .{ }^{13} \mathrm{C}$ NMR $(101 \mathrm{MHz}$, $\left.\mathrm{CDCl}_{3}\right) \delta 159.2,132.4,131.6,130.6,128.9,127.6,122.7,114.1,55.4,31.9,29.9,29.1$, 28.1, 22.8, 14.3. HRMS-EI $(\mathrm{m} / \mathrm{z})$ : Calcd for $\mathrm{C}_{17} \mathrm{H}_{24} \mathrm{O}[\mathrm{M}]^{+}$244.1827; found: 244.1831. 


\section{References}

[1] Zell, T.; Langer, R.; Iron, M. A.; Konstantinovski, L.; Shimon, L. J. W.; Diskin-Posner, Y.; Leitus, G.; Balaraman, E.; Ben-David, Y.; Milstein, D., Synthesis, Structures, and Dearomatization by Deprotonation of Iron Complexes Featuring Bipyridine-based PNN Pincer Ligands. Inorg. Chem. 2013, 52, 9636-9649.

[2] Peng, D.; Zhang, Y.; Du, X.; Zhang, L.; Leng, X.; Walter, M. D.; Huang, Z., Phosphinite-Iminopyridine Iron Catalysts for Chemoselective Alkene Hydrosilylation. J. Am. Chem. Soc. 2013, 135, 19154-19166.

[3] Zuo, Z.; Yang, J.; Huang, Z., Cobalt-Catalyzed Alkyne Hydrosilylation and Sequential Vinylsilane Hydroboration with Markovnikov Selectivity. Angew. Chem. Int. Ed. 2016, 55, 10839-10843.

[4] Zuo, Z.; Zhang, L.; Leng, X.; Huang, Z., Iron-catalyzed asymmetric hydrosilylation of ketones. Chem. Commun. 2015, 51, 5073-5076.

[5] Puenner, F.; Hilt, G., Regioselective solvent-dependent benzannulation of conjugated enynes. Chem. Commun. 2012, 48, 3617-3619.

[6] Zhang, Y.; Yu, B.; Gao, B.; Zhang, T.; Huang, H., Triple-Bond Insertion Triggers Highly Regioselective 1,4-Aminomethylamination of 1,3-Enynes with Aminals Enabled by Pd-Catalyzed C-N Bond Activation. Org. Lett. 2019, 21, 535-539.

[7] Cheng, J.-K.; Loh, T.-P., Copper- and Cobalt-Catalyzed Direct Coupling of sp(3) alpha-Carbon of Alcohols with Alkenes and Hydroperoxides. J. Am. Chem. Soc. 2015, 137, 42-45.

[8] Wang, F.; Wang, D.; Zhou, Y.; Liang, L.; Lu, R.; Chen, P.; Lin, Z.; Liu, G., Divergent Synthesis of CF3-Substituted Allenyl Nitriles by Ligand-Controlled Radical 1,2-and 1,4-Addition to 1,3-Enynes. Angew. Chem. Int. Ed. 2018, 57, 7140-7145.

[9] Buszek, K. R., FIRST INTRAMOLECULAR BENZYNE DIELS-ALDER REACTION WITH AN ACYCLIC DIENE - UNUSUAL EFFECT OF DIENE GEOMETRY ON THE COURSE OF THE REACTION. Tetrahedron Lett. 1995, 36, 9125-9128.

[10] Feuerstein, M.; Chahen, L.; Doucet, H.; Santelli, M., Efficient synthesis of enynes by tetraphosphine-palladium-catalysed reaction of vinyl bromides with terminal alkynes. Tetrahedron 2006, 62, 112-120.

[11] Schabel, T.; Plietker, B., Microwave-Accelerated Ru-Catalyzed Hydrovinylation of Alkynes and Enynes: A Straightforward Approach toward 1,3-Dienes and 1,3,5-Trienes. Chem. Eur. J. 2013, 19, 6938-6941.

[12]Lin, Y.-Y.; Wang, Y.-J.; Cheng, J.-H.; Lee, C.-F., Copper-Catalyzed Coupling of Alkynes with Alkenyl Halides. Synlett 2012, 930-934.

[13]a)Du, X.; Hou, W.; Zhang, Y.; Huang, Z., Pincer cobalt complex-catalyzed Z-selective hydrosilylation of terminal alkynes. Org. Chem. Front. 2017, 4, 1517-1521; b)Hu, M.-Y.; He, P.; Qiao, T.-Z.; Sun, W.; Li, W.-T.; Lian, J.; Li, J.-H.; Zhu, S.-F., Iron-Catalyzed Regiodivergent Alkyne Hydrosilylation. J. Am. Chem. Soc. 
2020, 142, 16894-16902.

[14]Wen, H.; Wang, K.; Zhang, Y.; Liu, G.; Huang, Z., Cobalt-Catalyzed Regio- and Enantioselective Markovnikov 1,2-Hydrosilylation of Conjugated Dienes. ACS Catal. 2019, 9, 1612-1618.

[15]Zhou, H.; Moberg, C., Regio- and Stereoselective Hydrosilylation of 1,3-Enynes Catalyzed by Palladium. Org. Lett. 2013, 15, 1444-1447. 


\section{Copies of NMR Spectra}

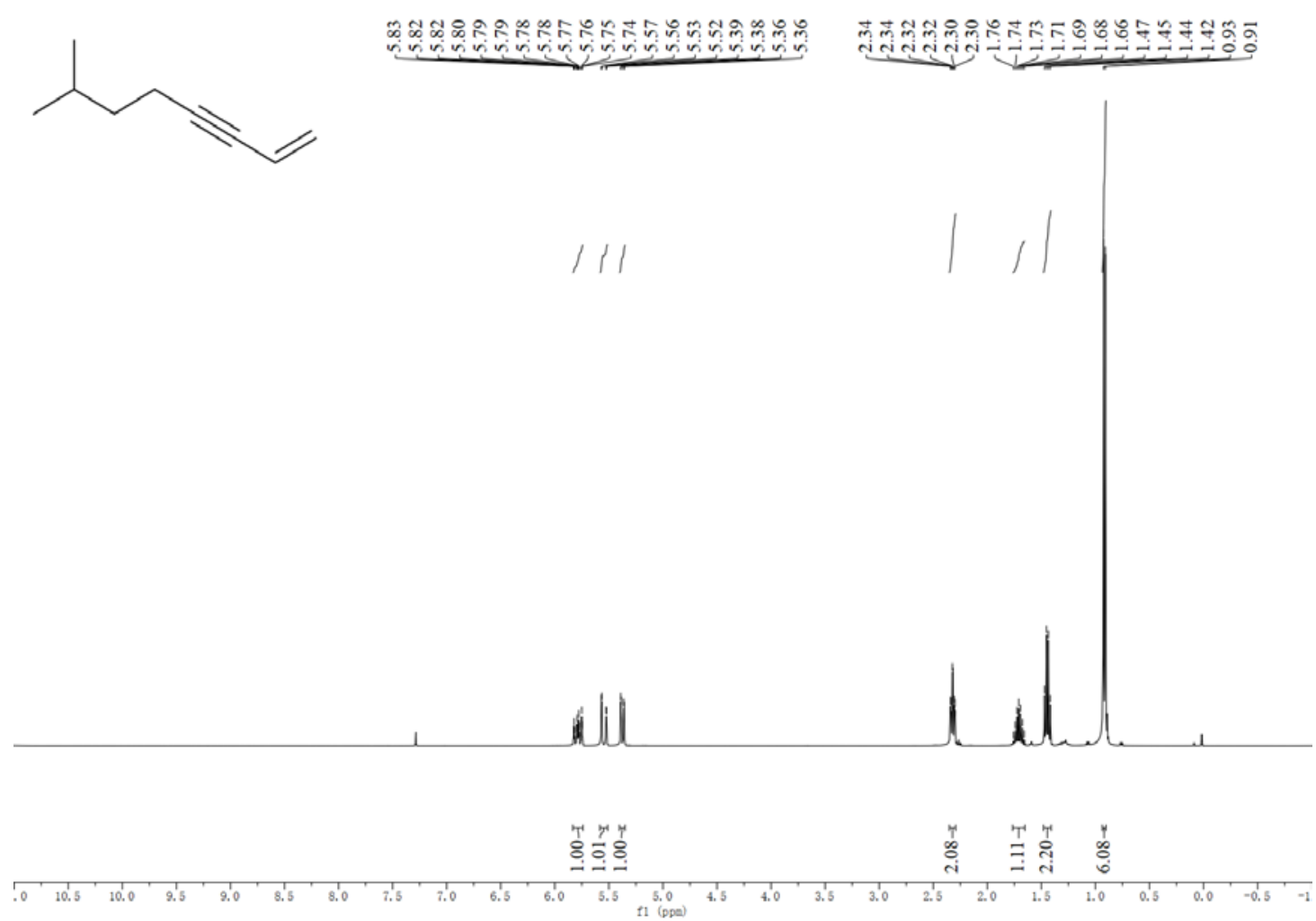

${ }^{1} \mathrm{H}$ NMR (400 MHz, $\mathrm{CDCl}_{3}$ ) spectrum of $\mathbf{2 e}$
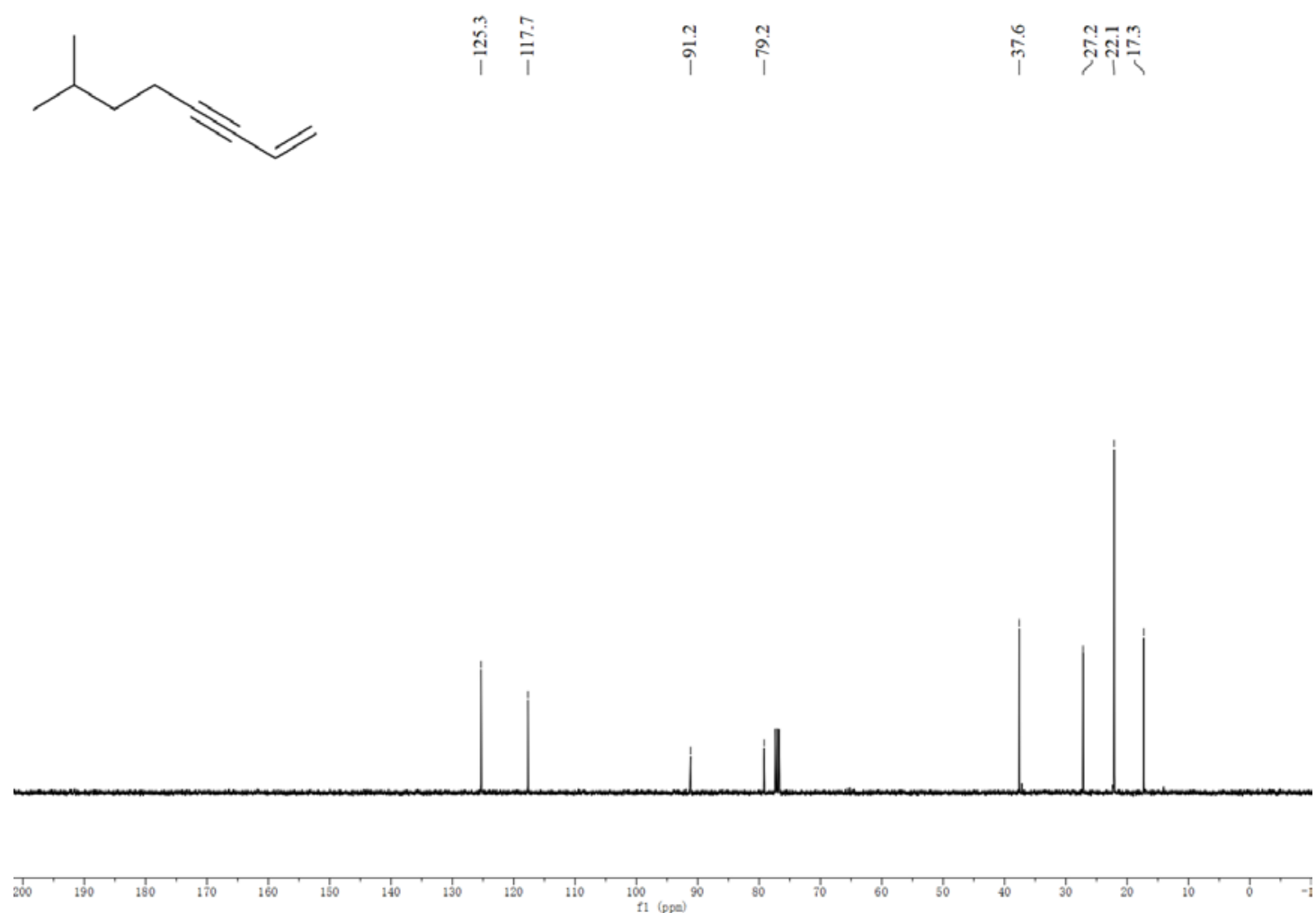

${ }^{13} \mathrm{C}$ NMR (101 MHz, $\mathrm{CDCl}_{3}$ ) spectrum of $\mathbf{2 e}$ 

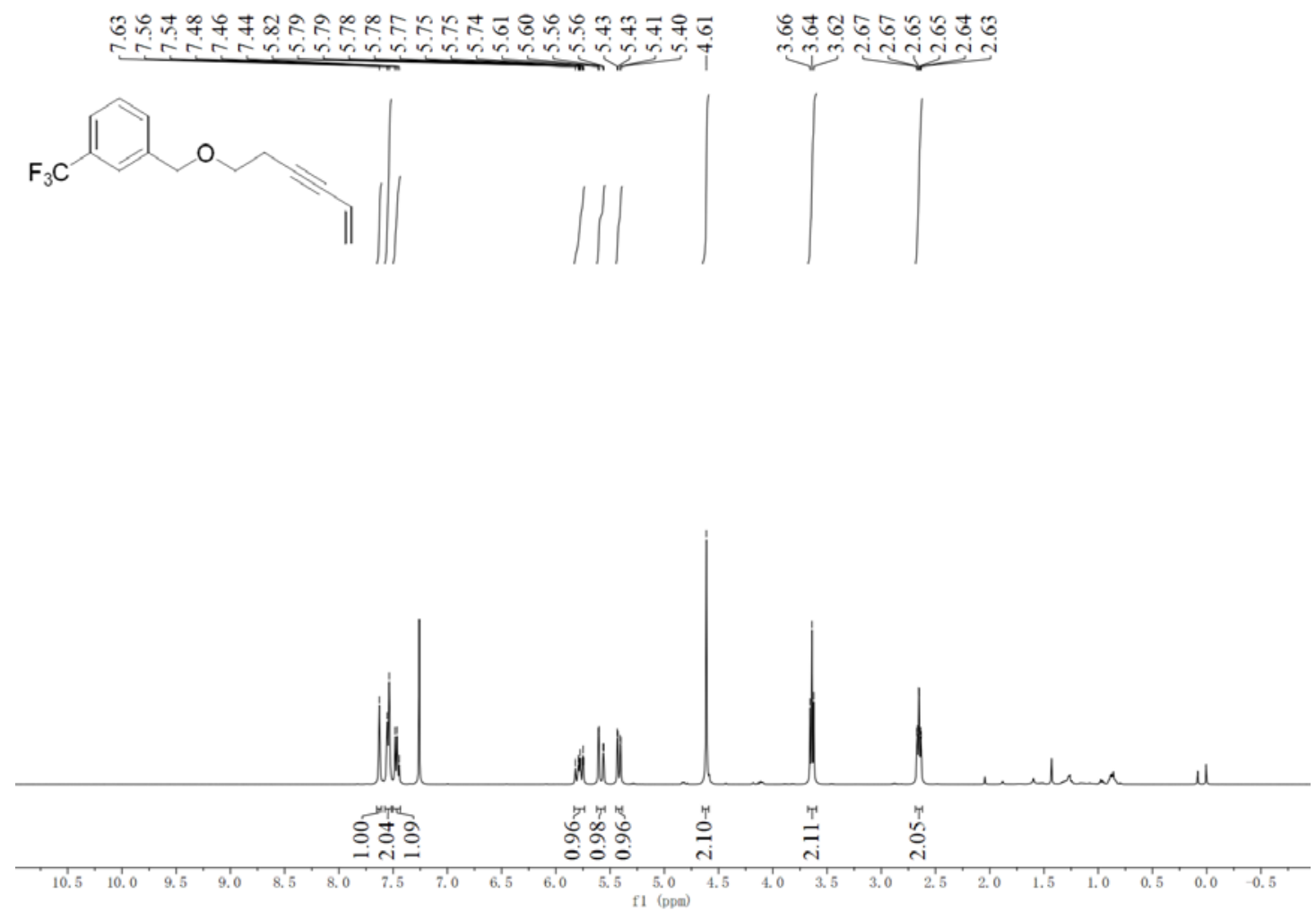

${ }^{1} \mathrm{H}$ NMR (400 MHz, $\mathrm{CDCl}_{3}$ ) spectrum of $\mathbf{2 i}$
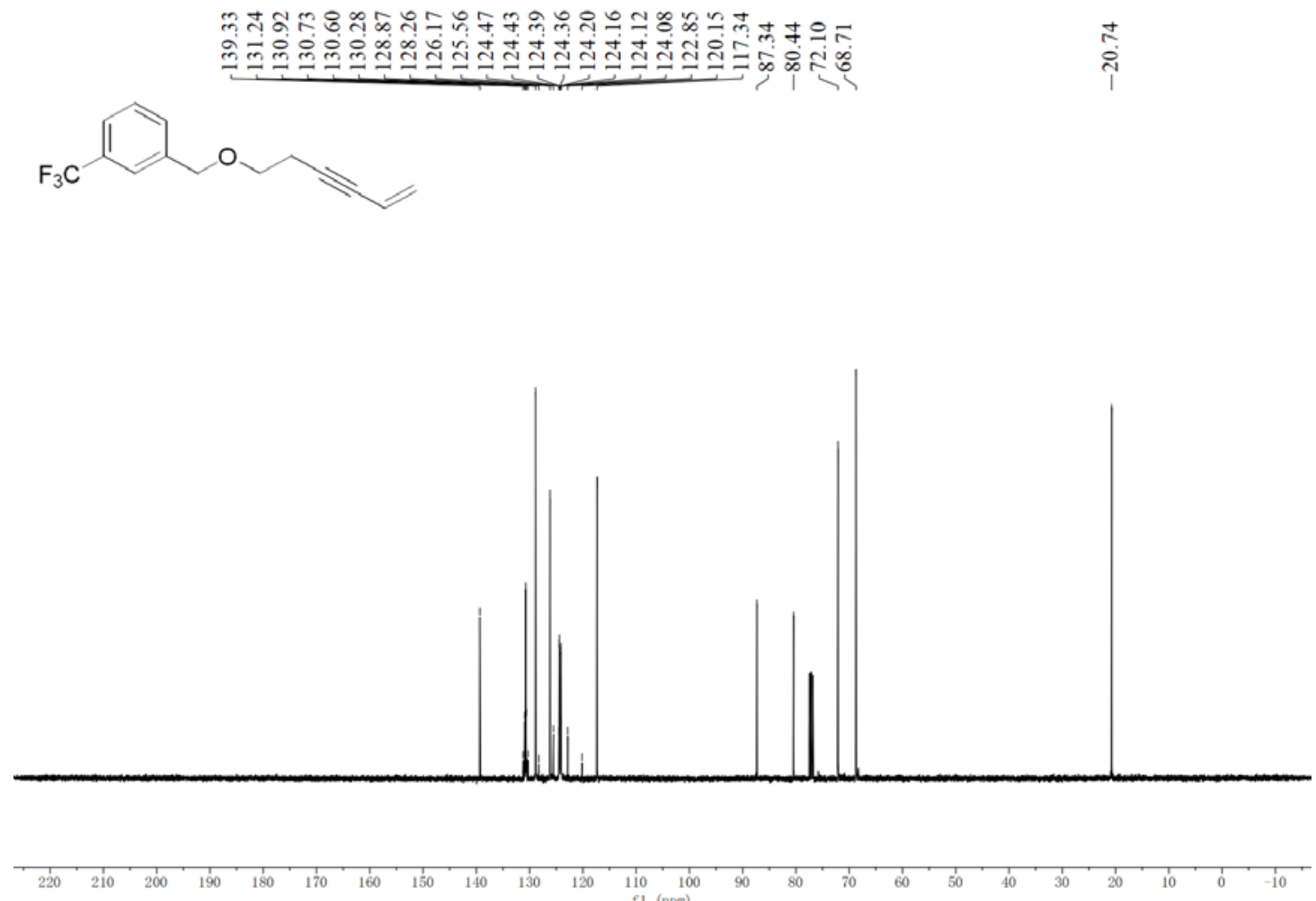

${ }^{13} \mathrm{C}$ NMR (101 MHz, $\mathrm{CDCl}_{3}$ ) spectrum of $\mathbf{2 i}$ 

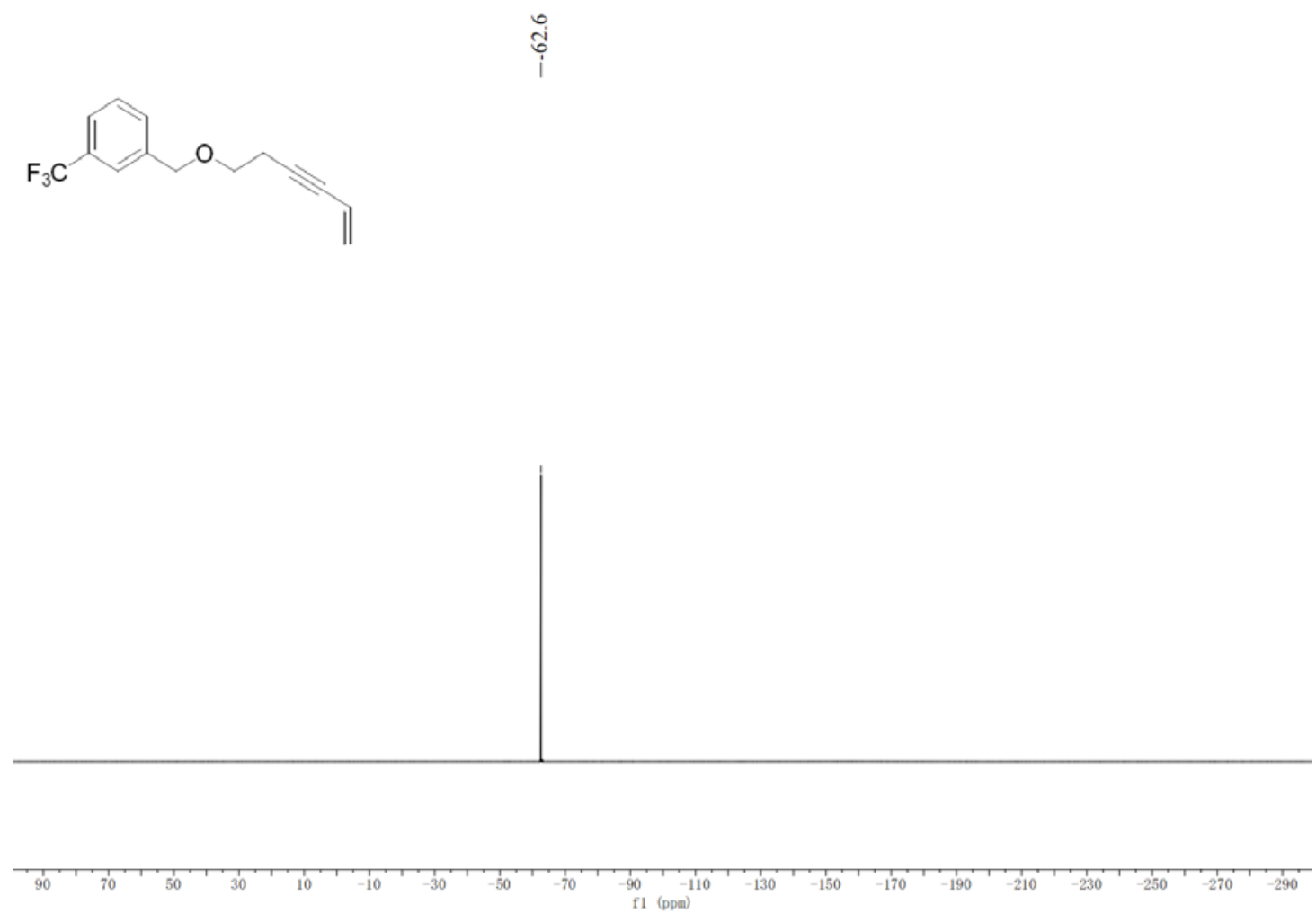

${ }^{19} \mathrm{~F}$ NMR (376 $\mathrm{MHz}, \mathrm{CDCl}_{3}$ ) spectrum of $\mathbf{2 i}$

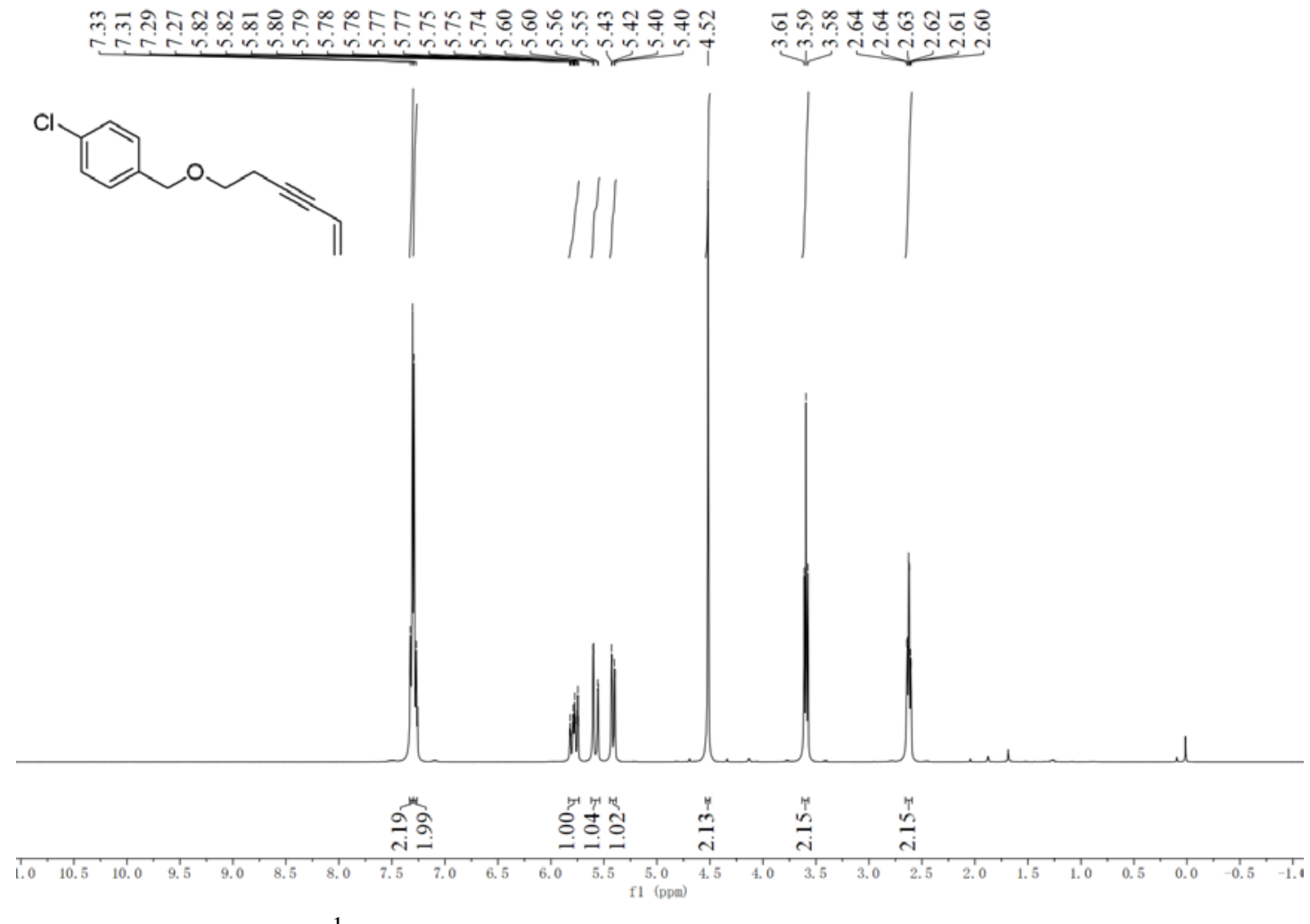

${ }^{1} \mathrm{H}$ NMR (400 MHz, $\mathrm{CDCl}_{3}$ ) spectrum of $\mathbf{2 j}$ 
<smiles>C#CC#CCCOCc1ccc(Cl)cc1</smiles>
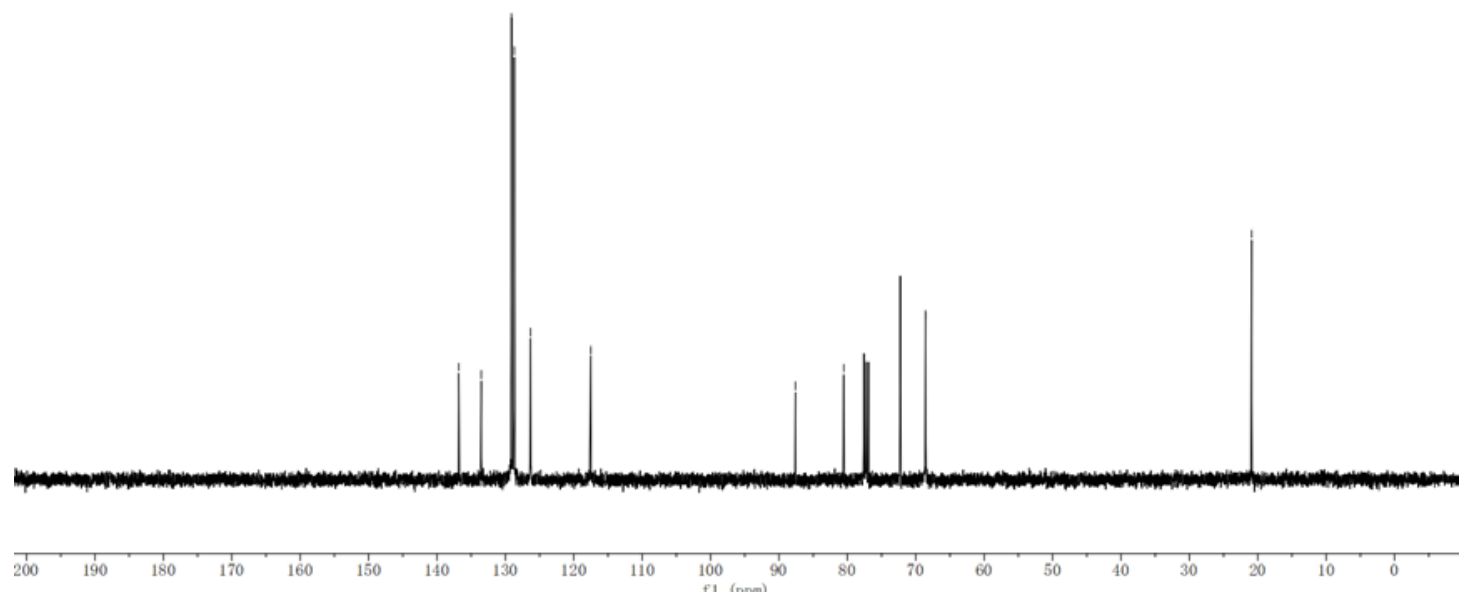

${ }^{13} \mathrm{C}$ NMR (101 MHz, $\left.\mathrm{CDCl}_{3}\right)$ spectrum of $\mathbf{2} \mathbf{j}$

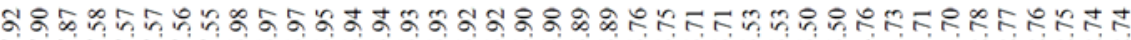
然
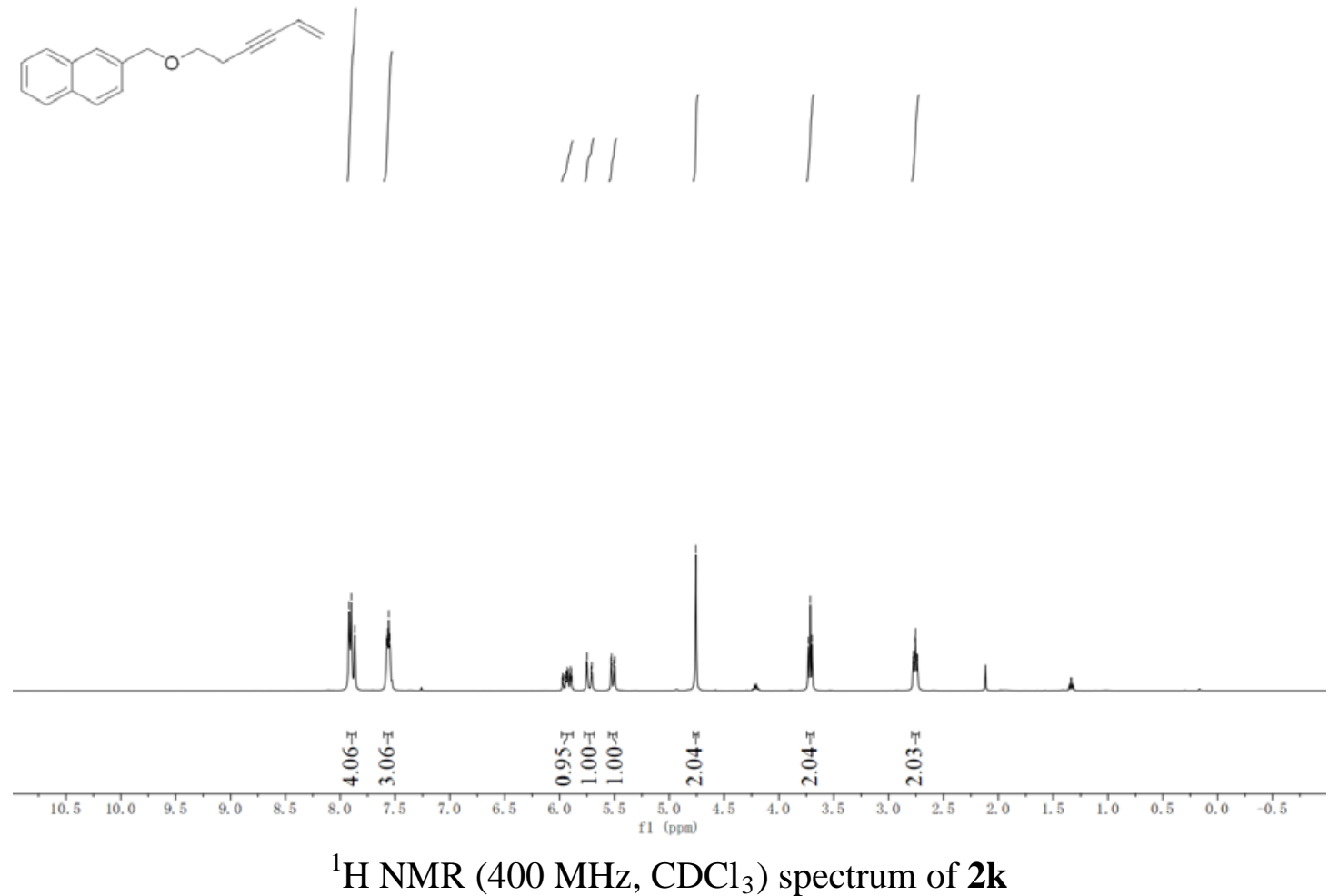

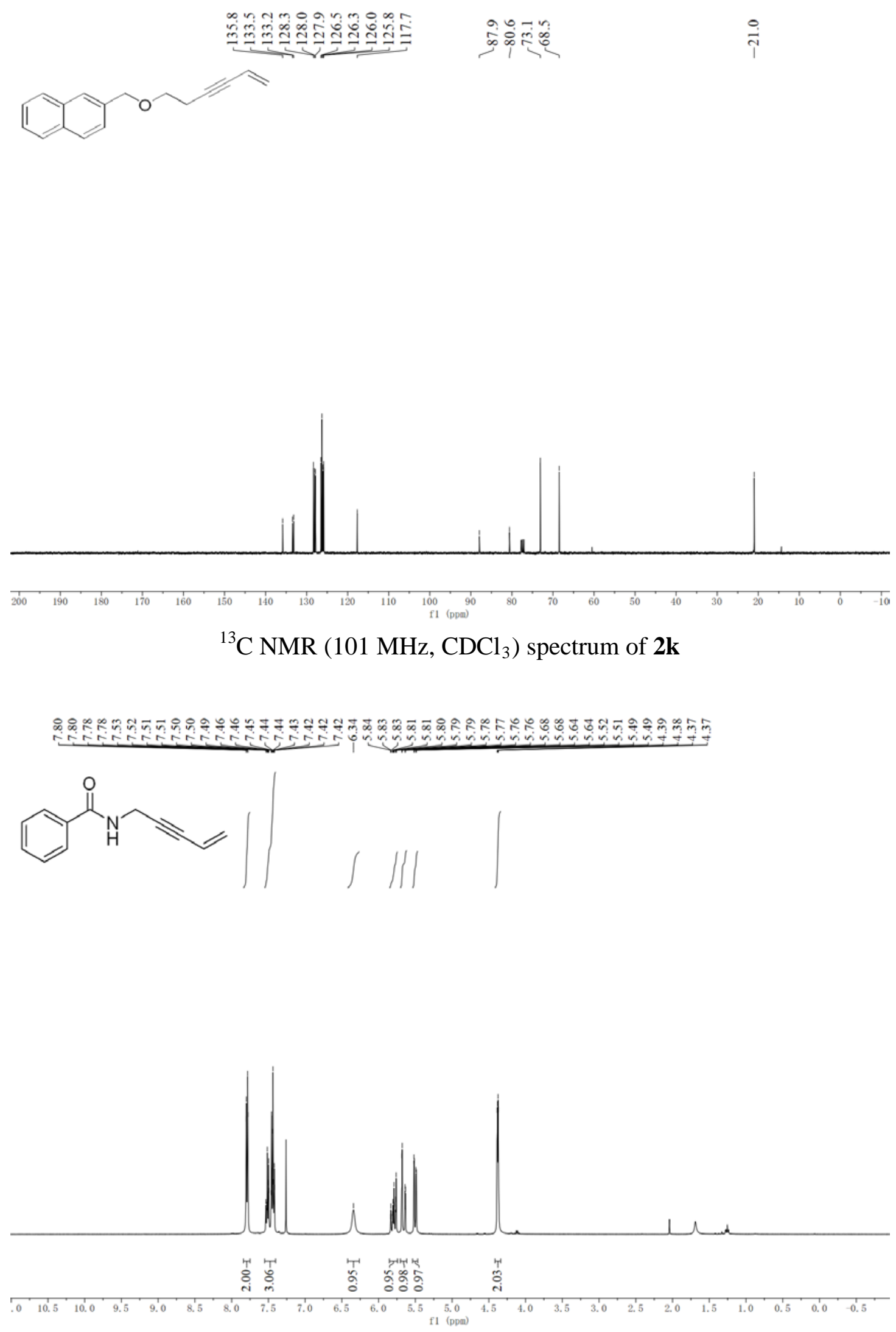

${ }^{1} \mathrm{H}$ NMR (400 MHz, $\mathrm{CDCl}_{3}$ ) spectrum of $2 \mathbf{l}$ 

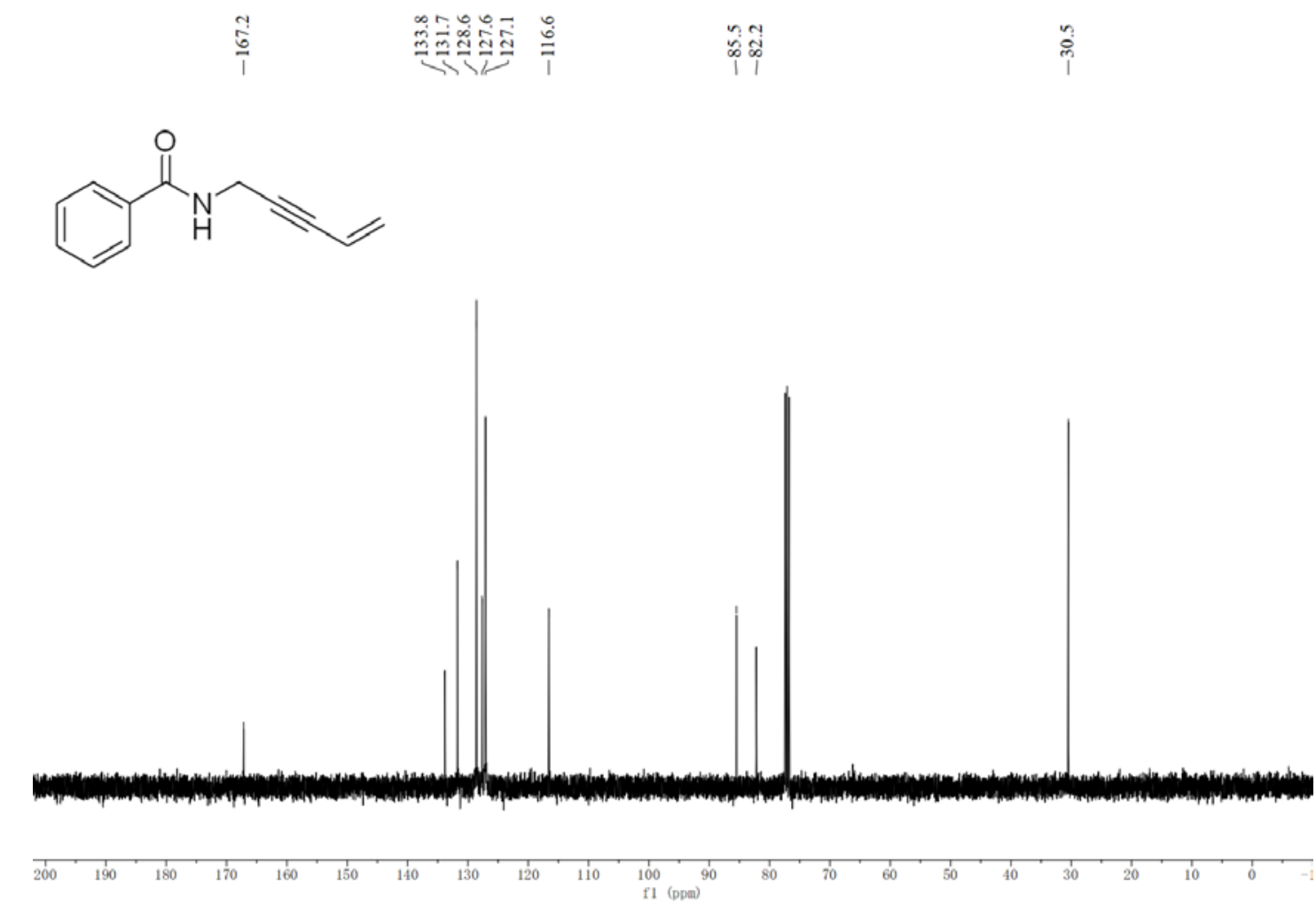

${ }^{13} \mathrm{C}$ NMR (101 MHz, $\left.\mathrm{CDCl}_{3}\right)$ spectrum of $2 \mathbf{l}$

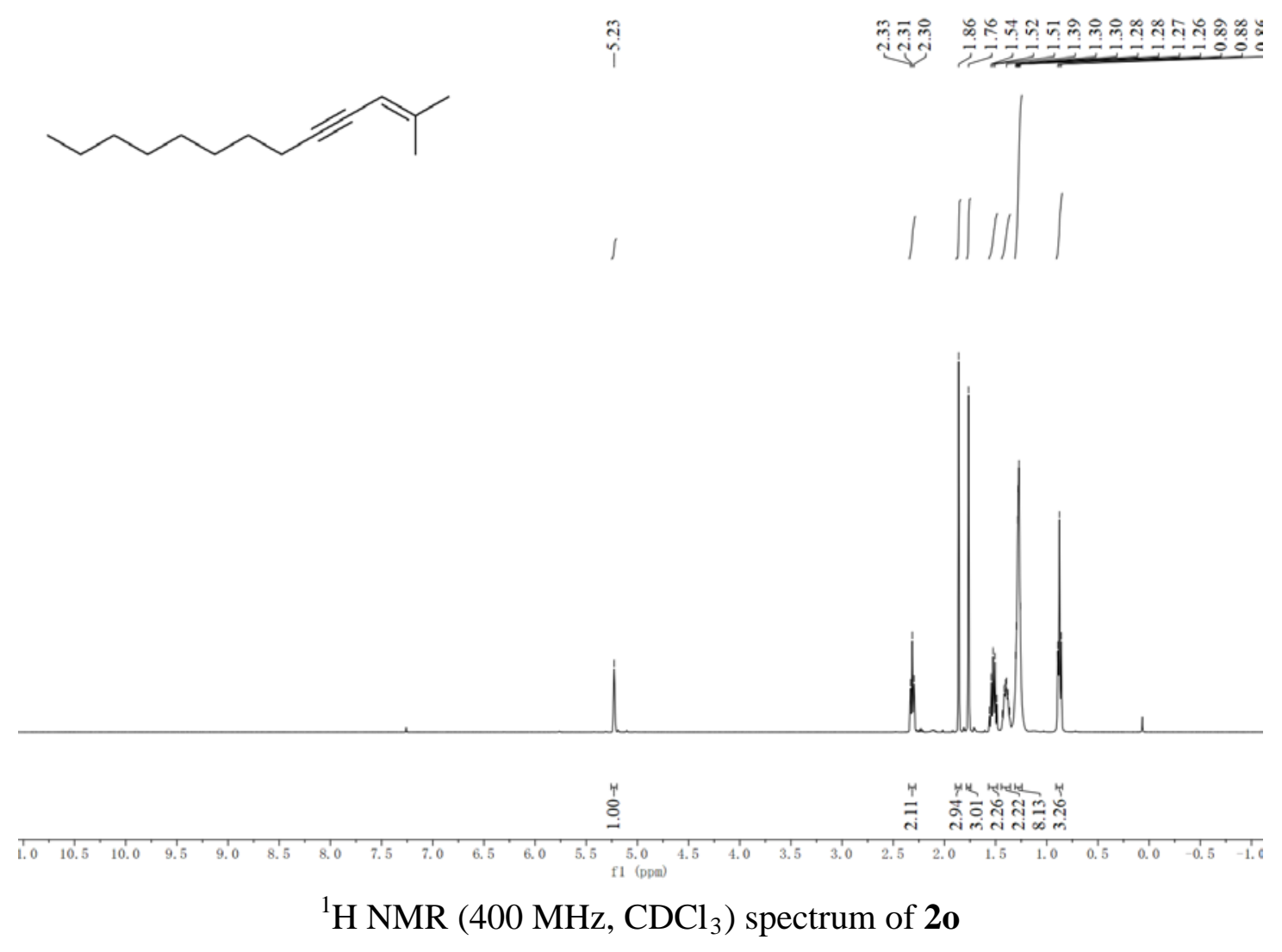



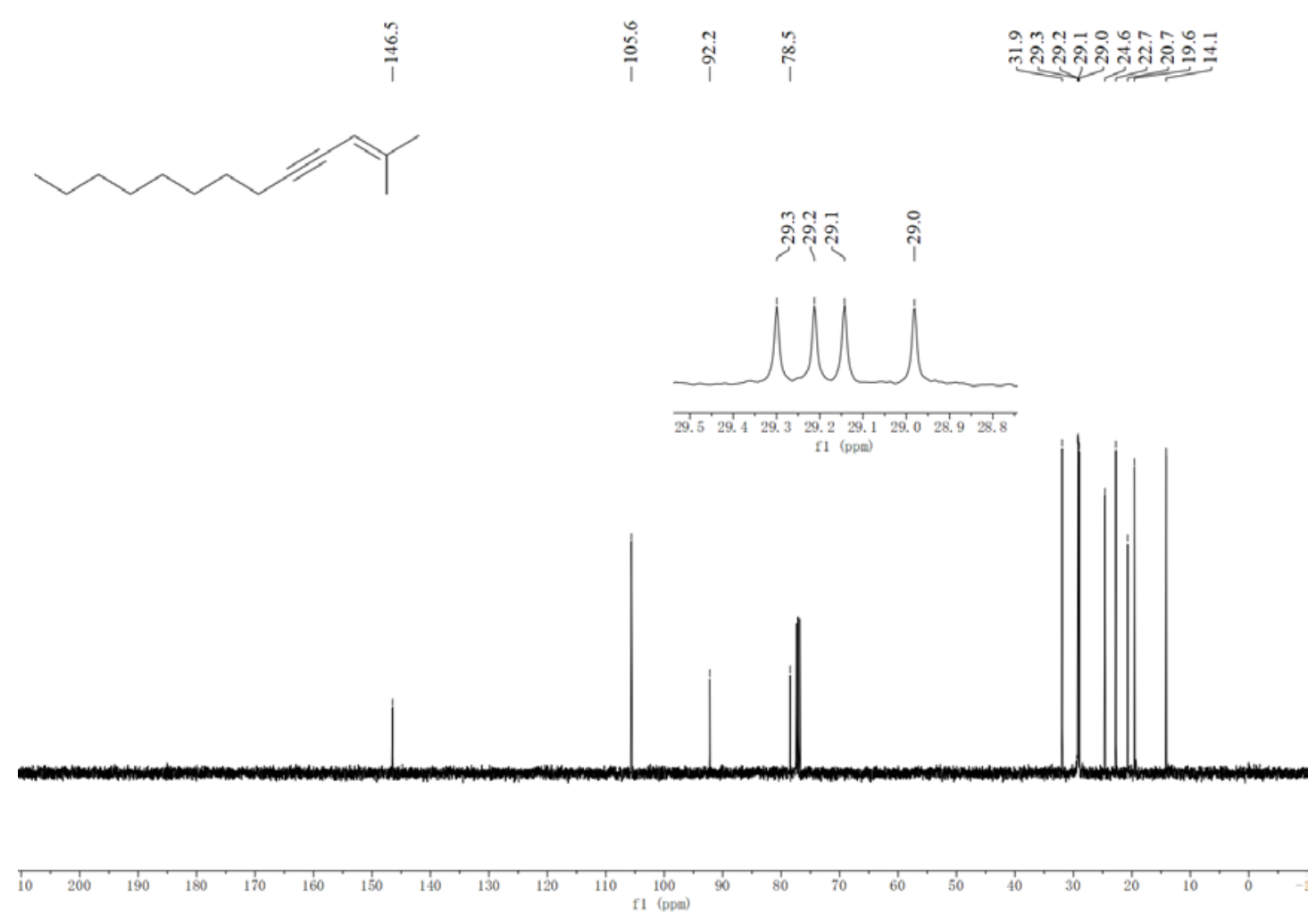

${ }^{13} \mathrm{C}$ NMR (101 MHz, $\mathrm{CDCl}_{3}$ ) spectrum of $2 \mathbf{o}$

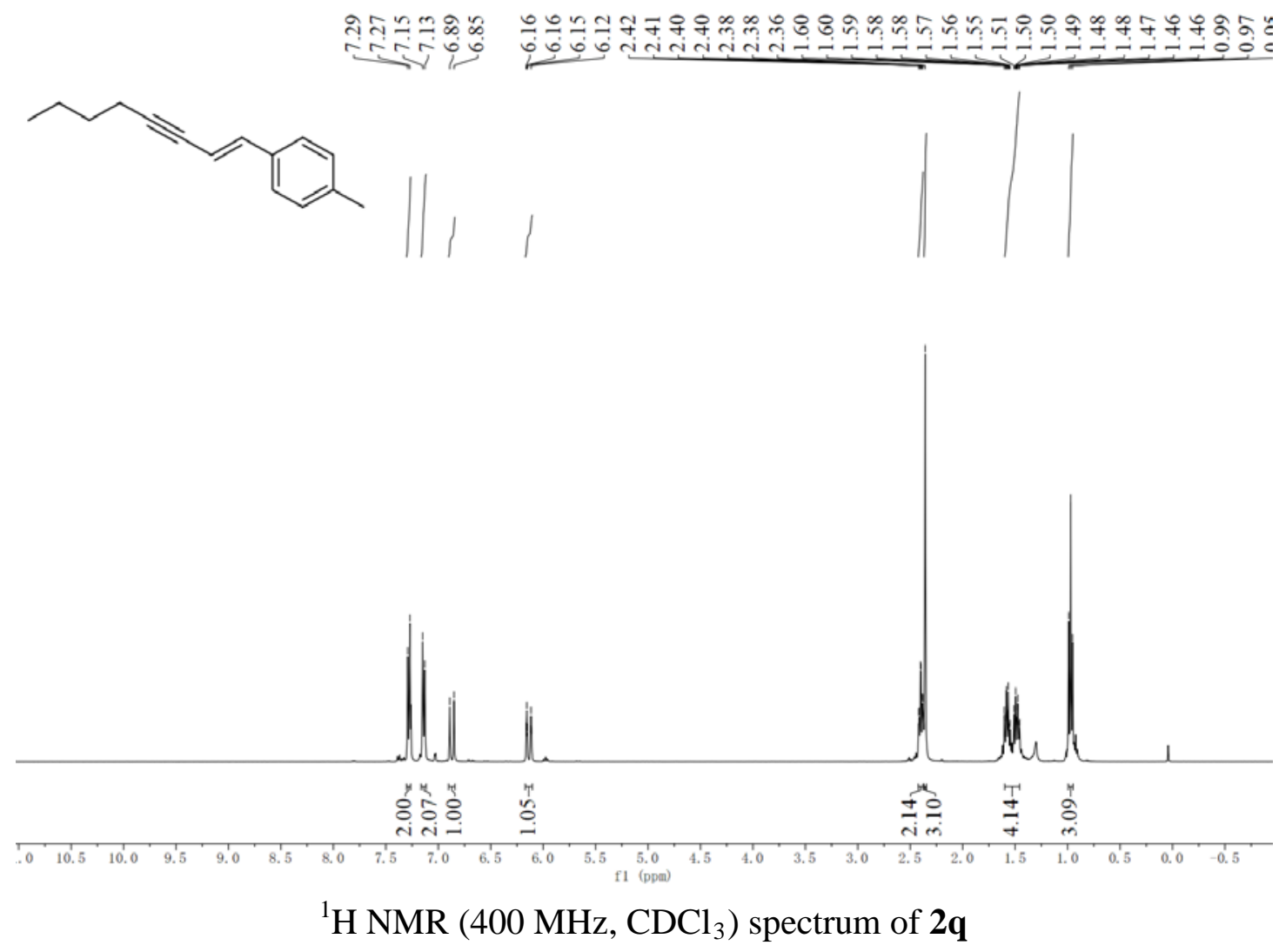




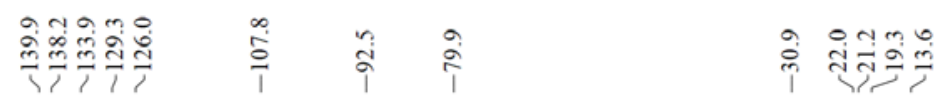

m的
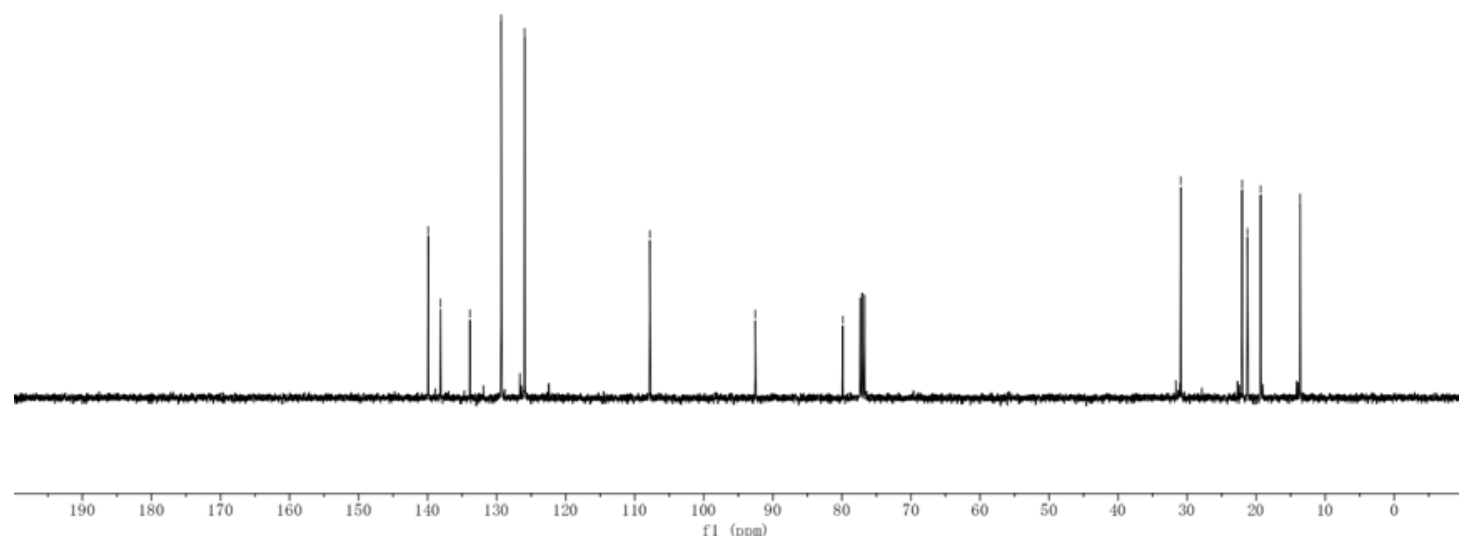

${ }^{13} \mathrm{C}$ NMR (101 MHz, $\mathrm{CDCl}_{3}$ ) spectrum of 2q

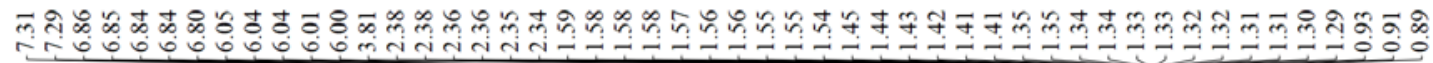

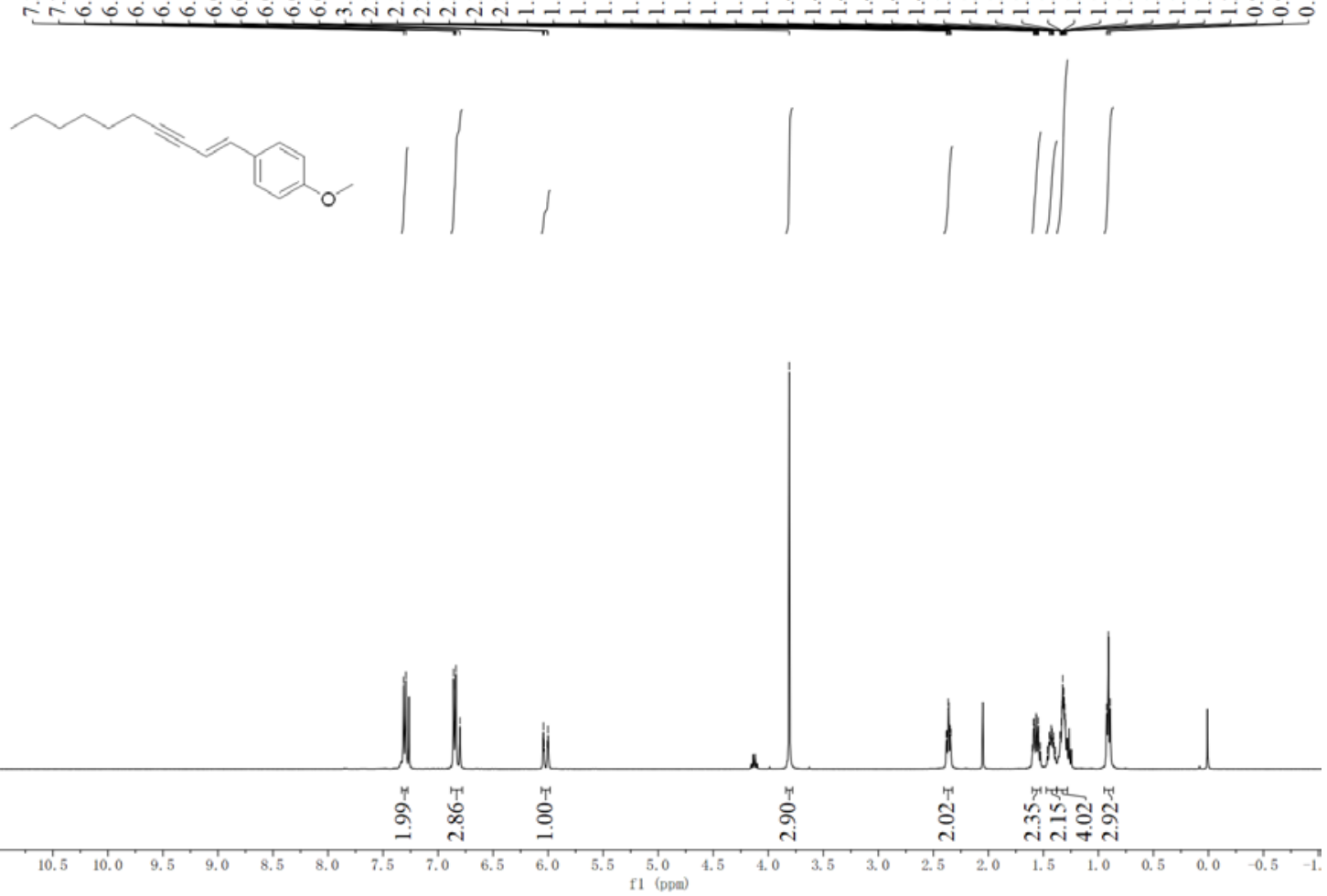

${ }^{1} \mathrm{H}$ NMR (400 MHz, $\mathrm{CDCl}_{3}$ ) spectrum of $\mathbf{2 r}$ 

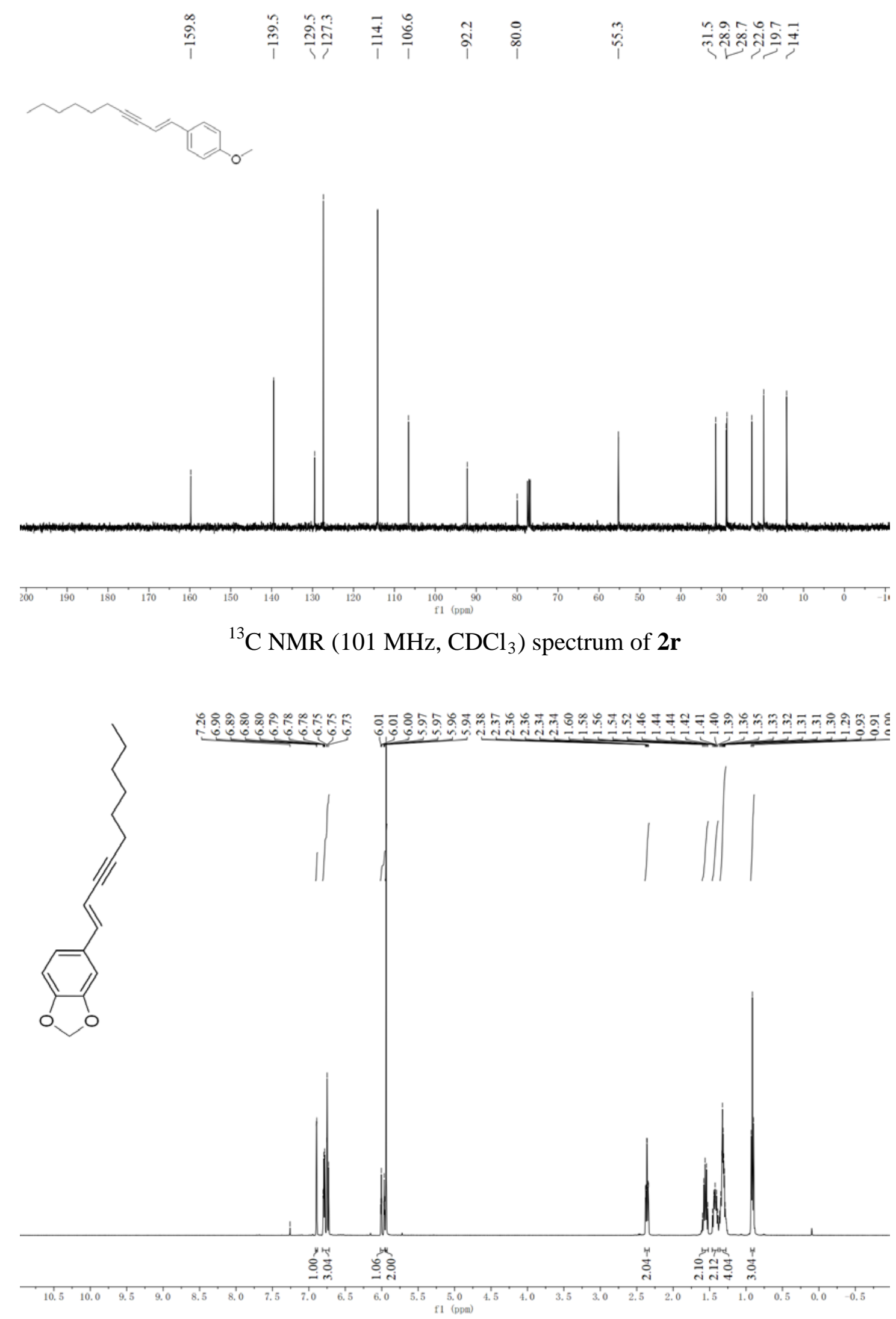

${ }^{1} \mathrm{H}$ NMR (400 MHz, $\mathrm{CDCl}_{3}$ ) spectrum of $2 \mathrm{~s}$ 

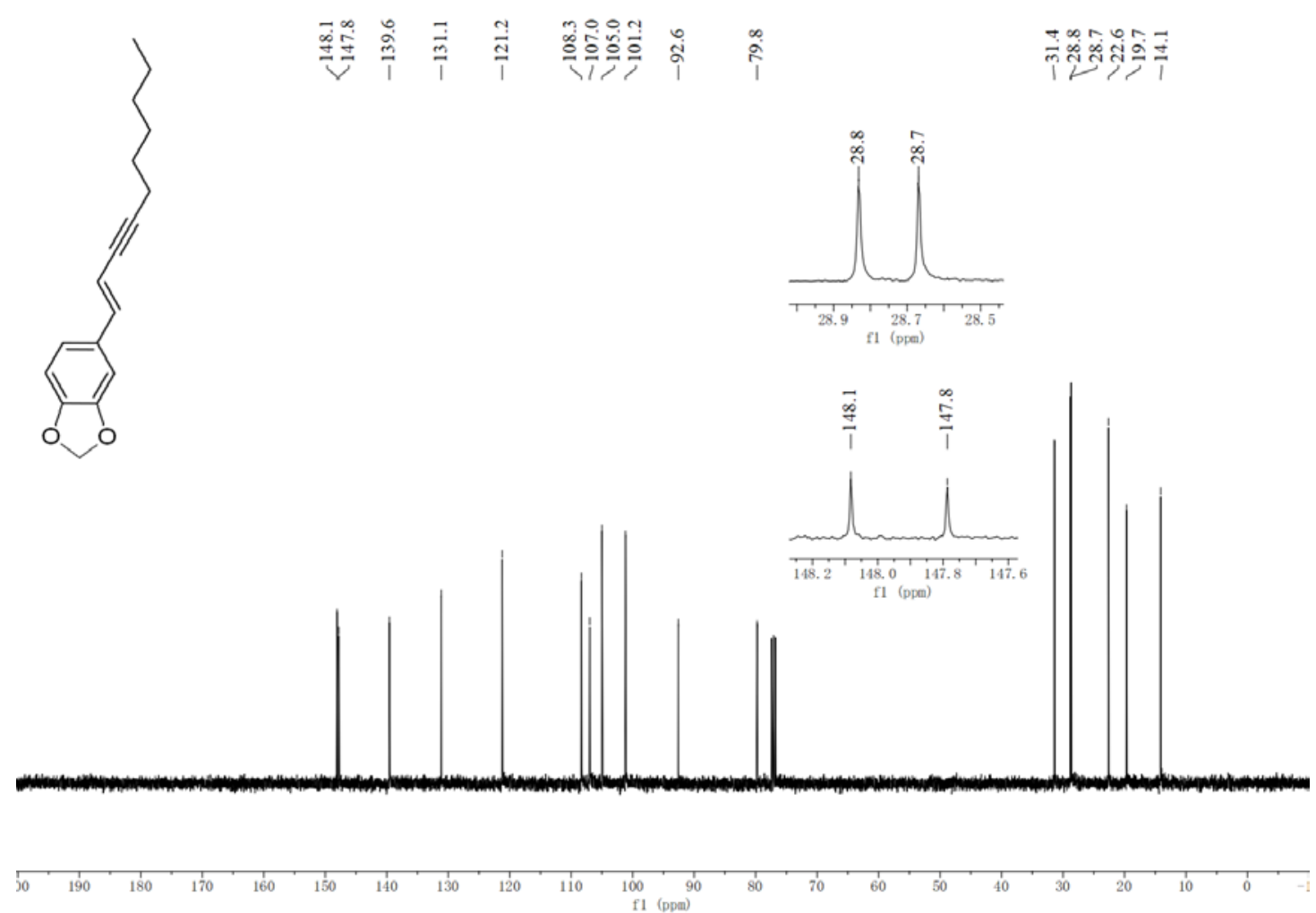

${ }^{13} \mathrm{C}$ NMR $\left(101 \mathrm{MHz}, \mathrm{CDCl}_{3}\right)$ spectrum of $2 \mathrm{~s}$
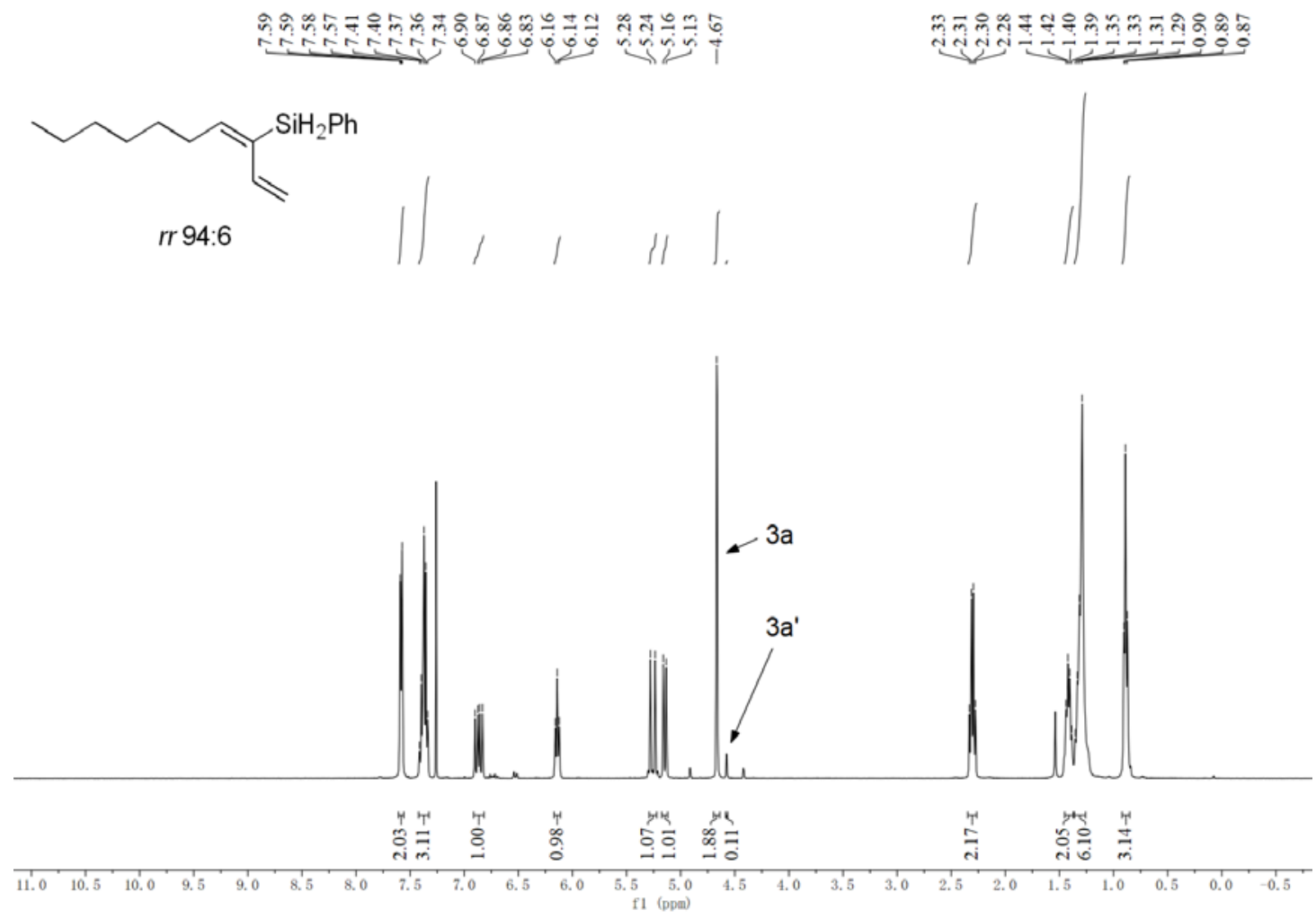

${ }^{1} \mathrm{H}$ NMR (400 MHz, $\mathrm{CDCl}_{3}$ ) spectrum of 3a 


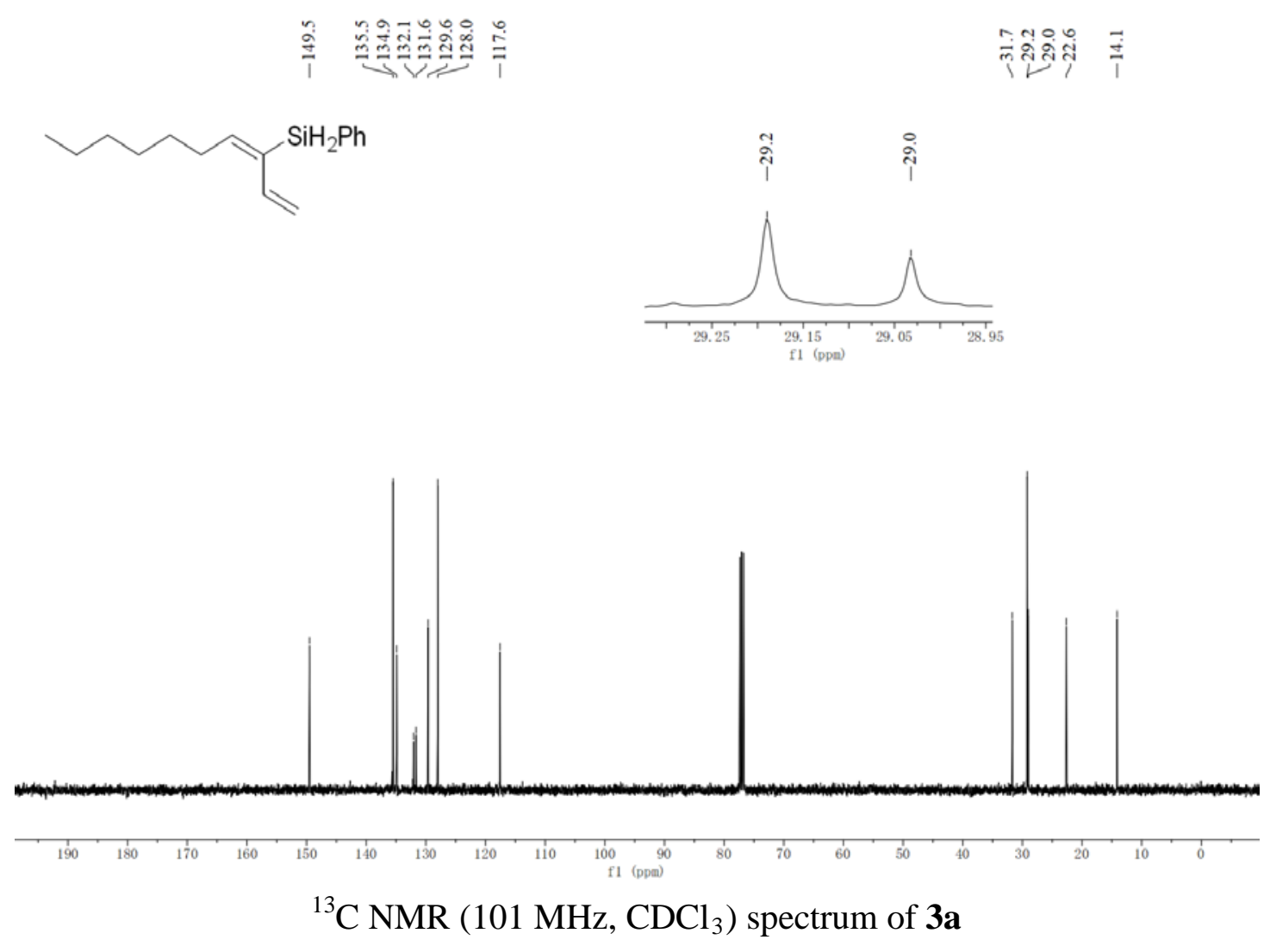




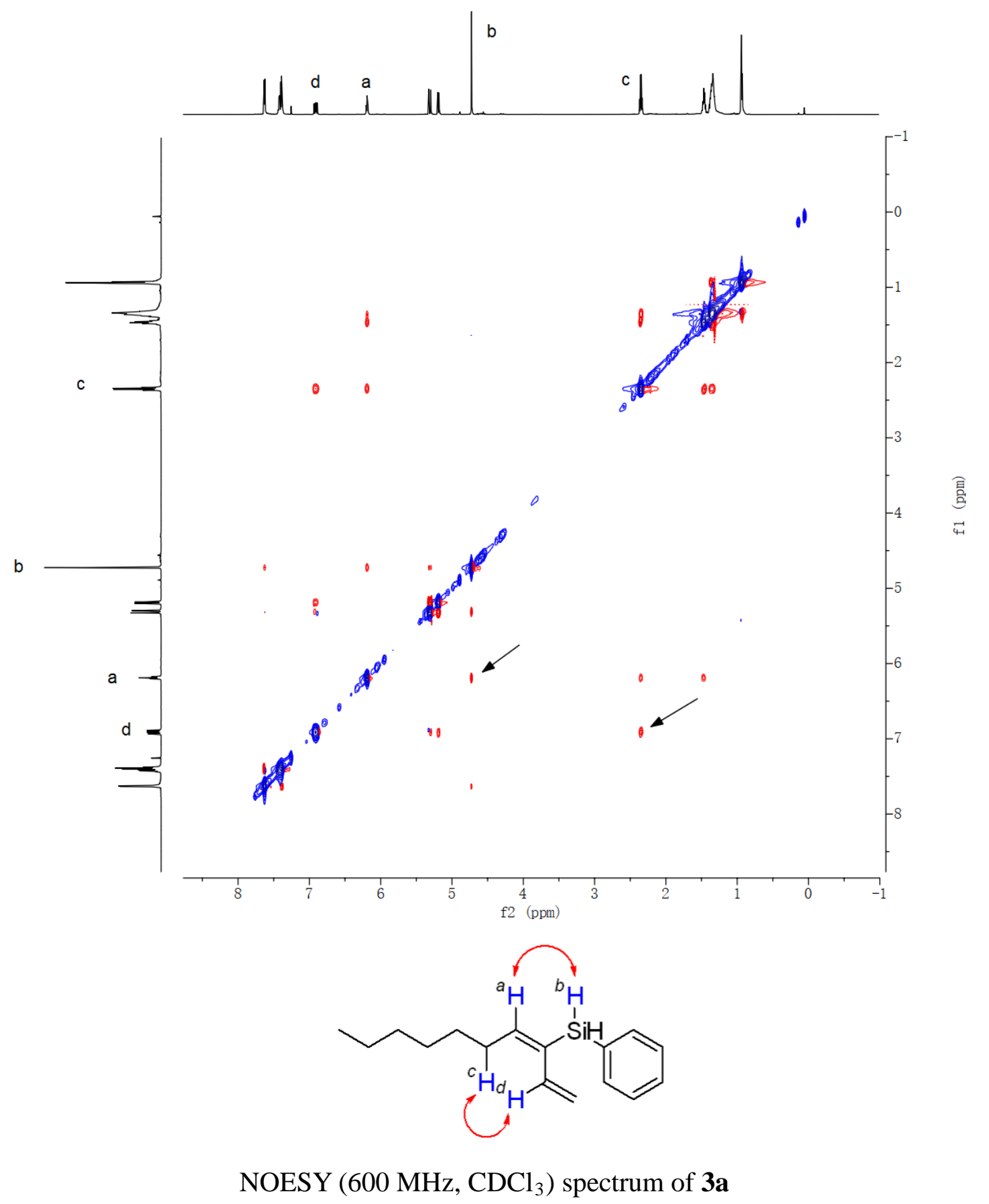




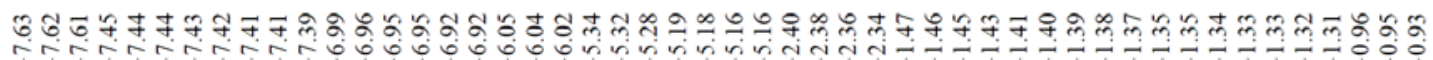
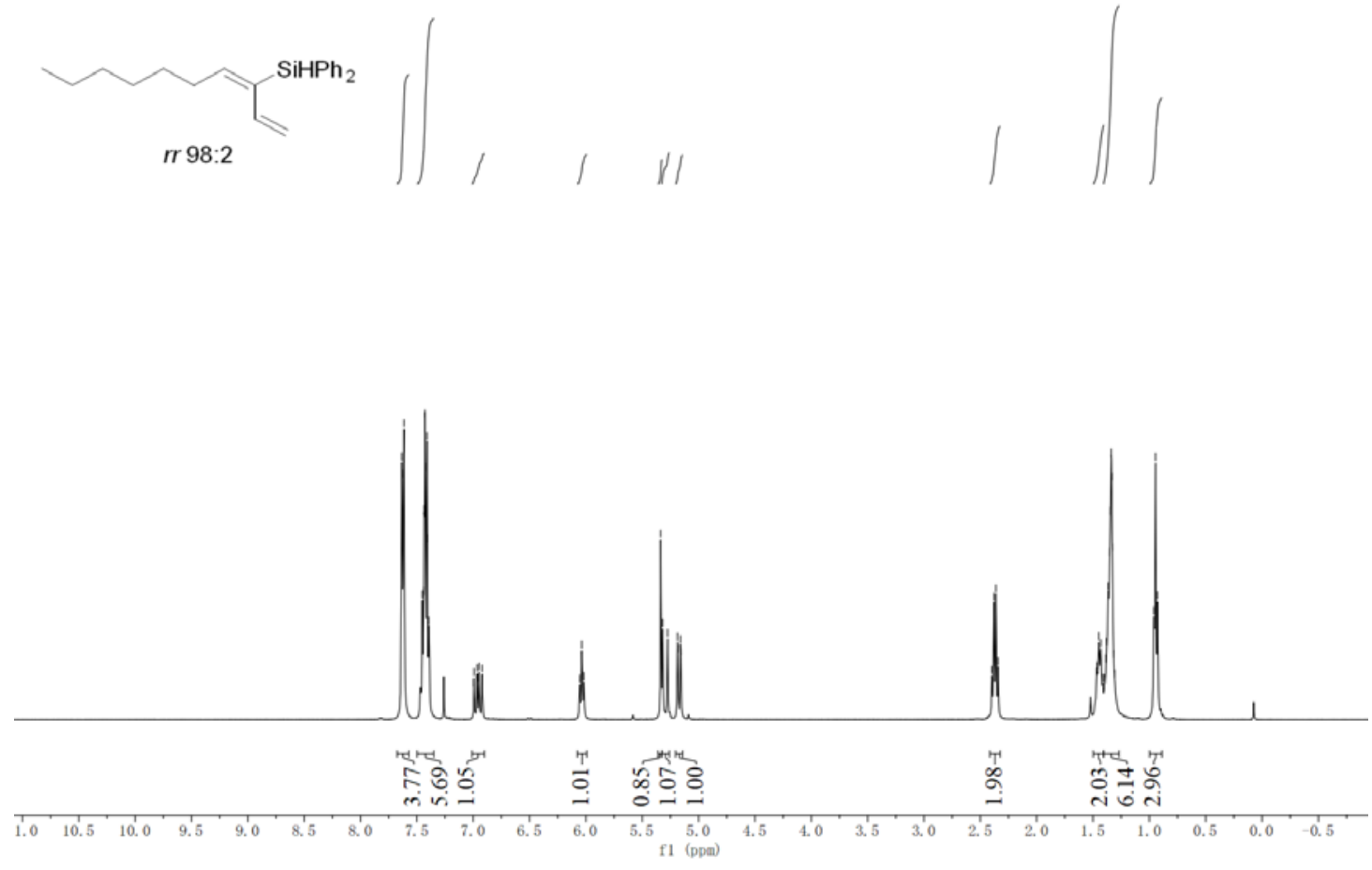

${ }^{1} \mathrm{H}$ NMR (400 MHz, $\mathrm{CDCl}_{3}$ ) spectrum of $\mathbf{3 b}$

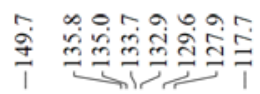

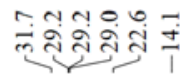
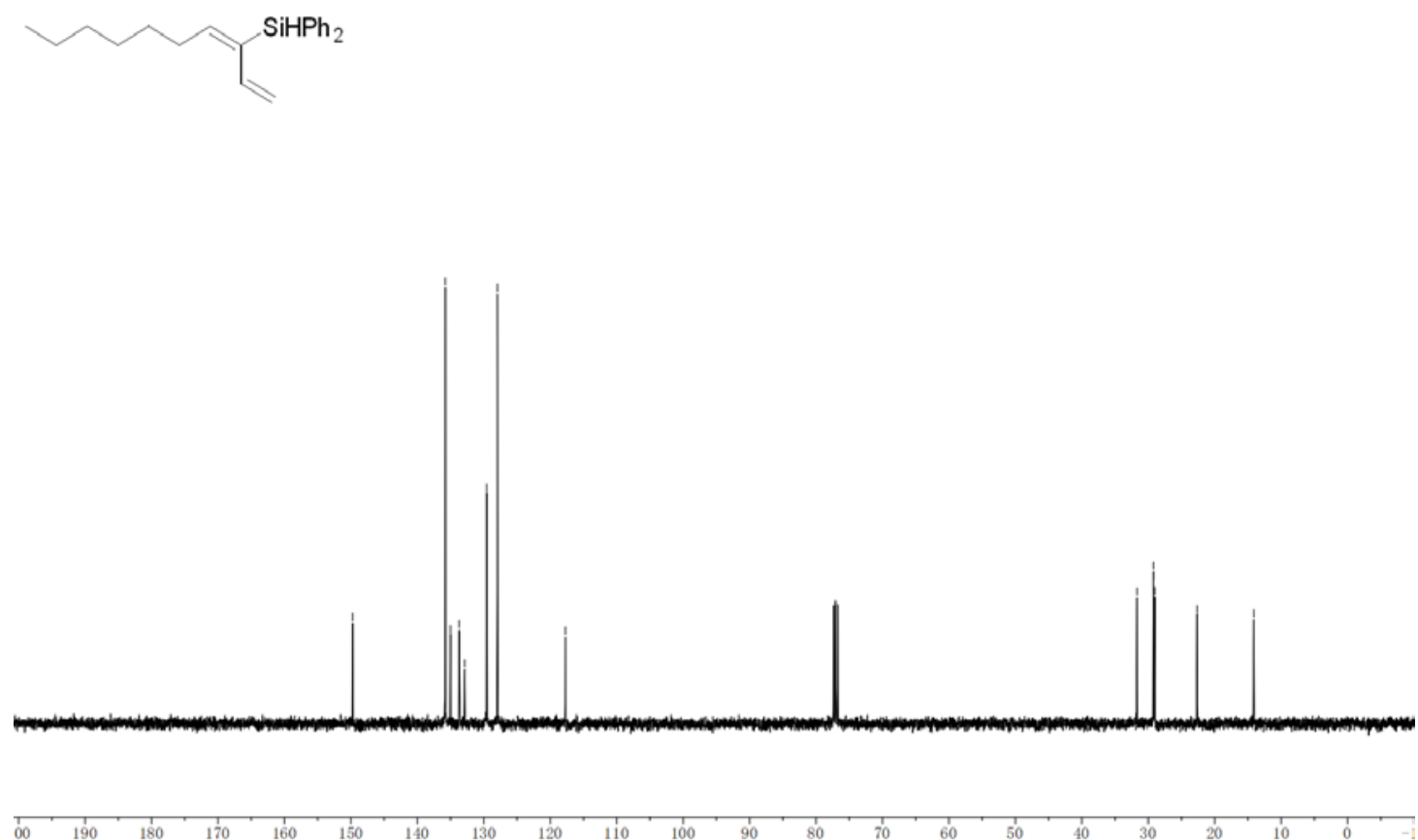

${ }^{13} \mathrm{C}$ NMR $\left(101 \mathrm{MHz}, \mathrm{CDCl}_{3}\right)$ spectrum of $\mathbf{3 b}$ 


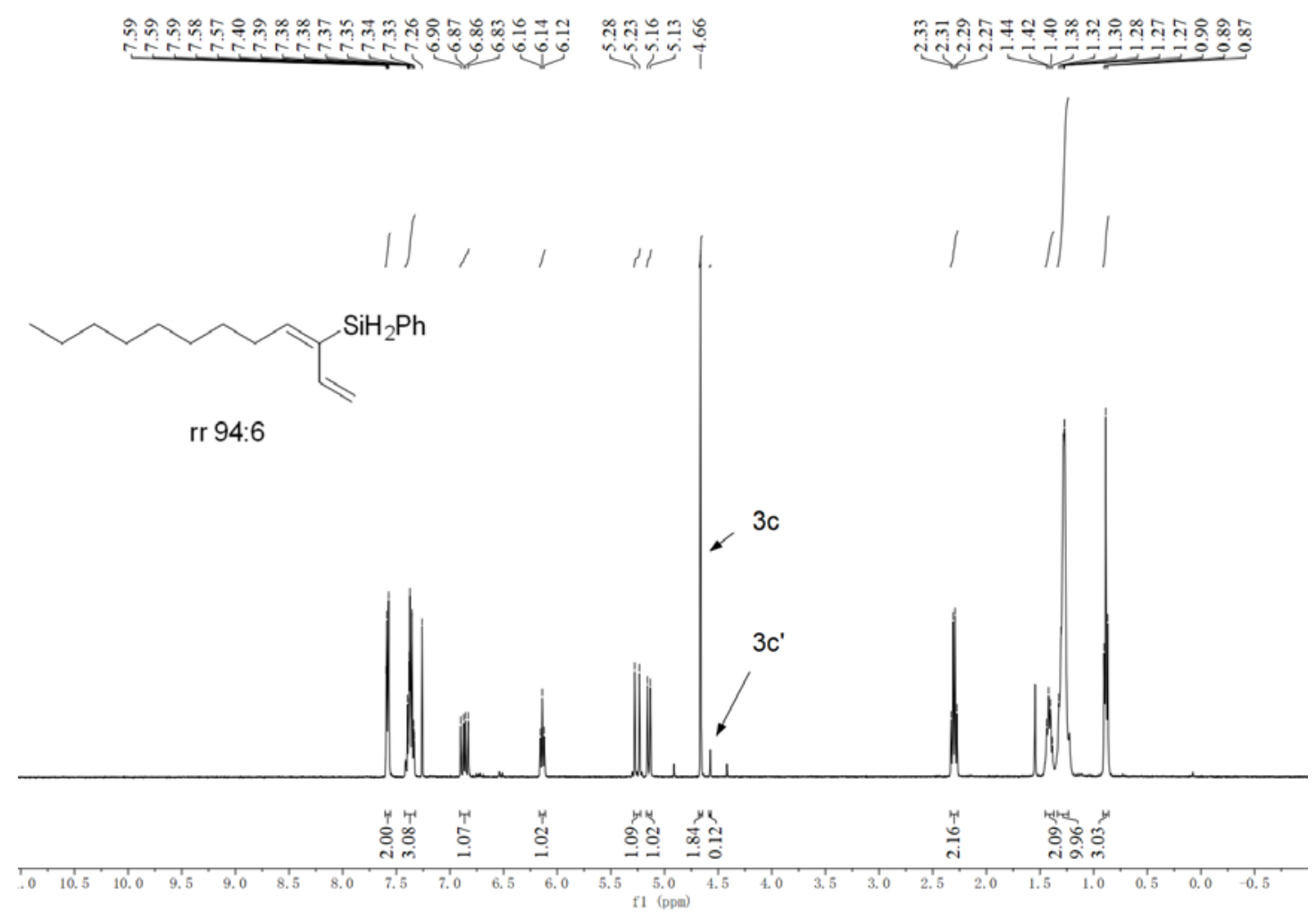

${ }^{1} \mathrm{H}$ NMR (400 MHz, $\mathrm{CDCl}_{3}$ ) spectrum of 3c

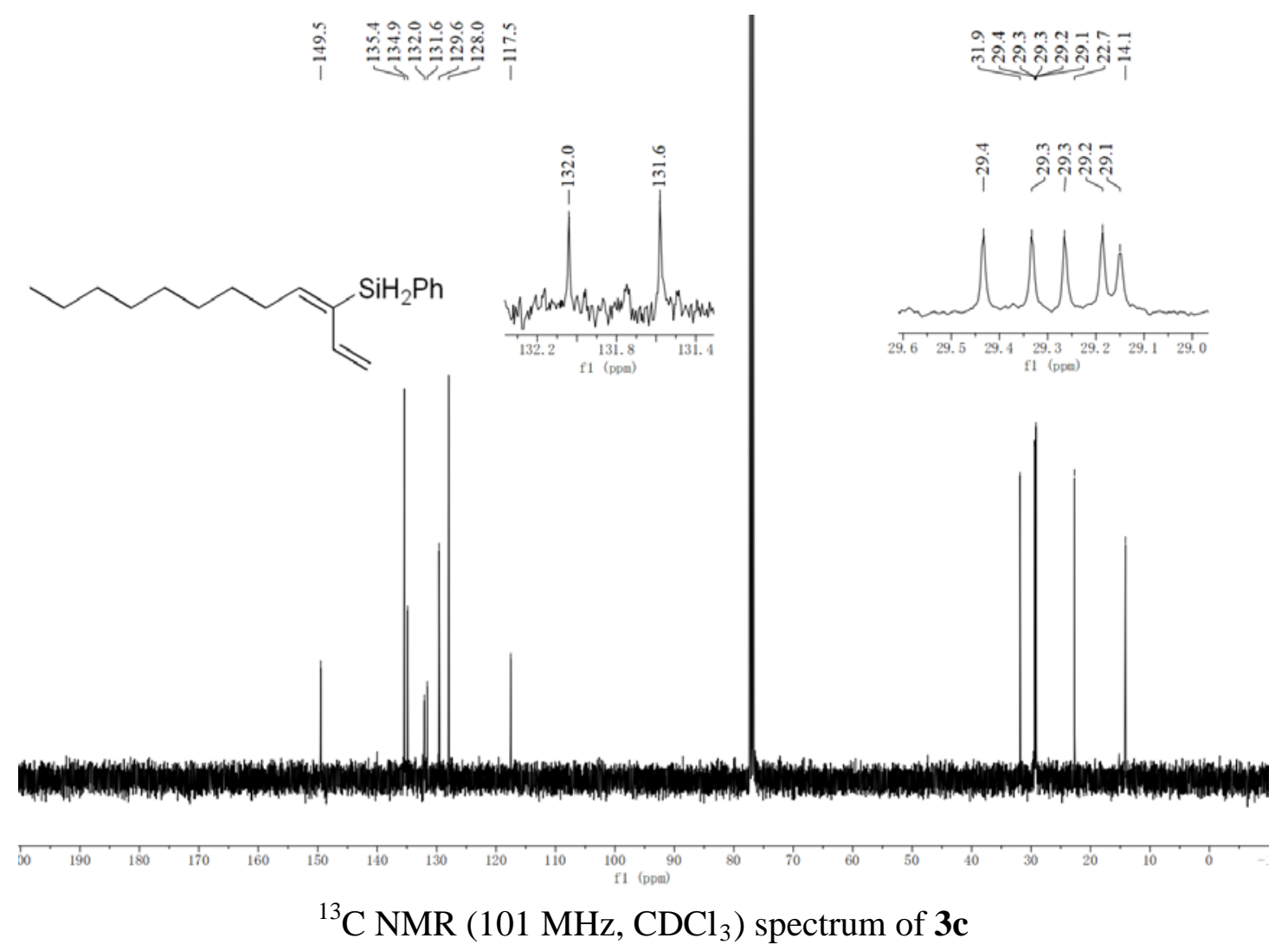



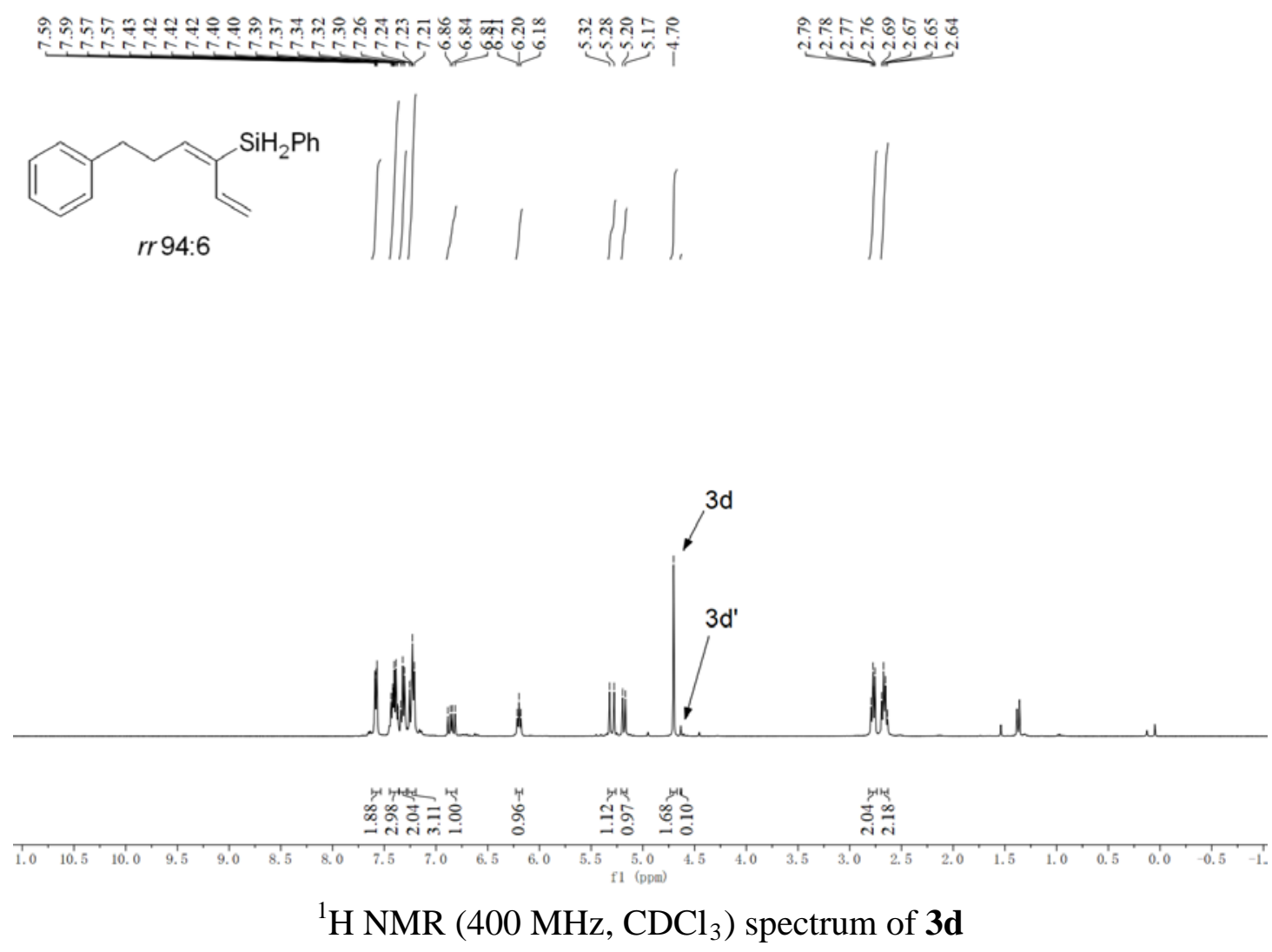

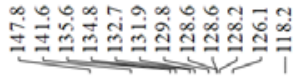<smiles></smiles>
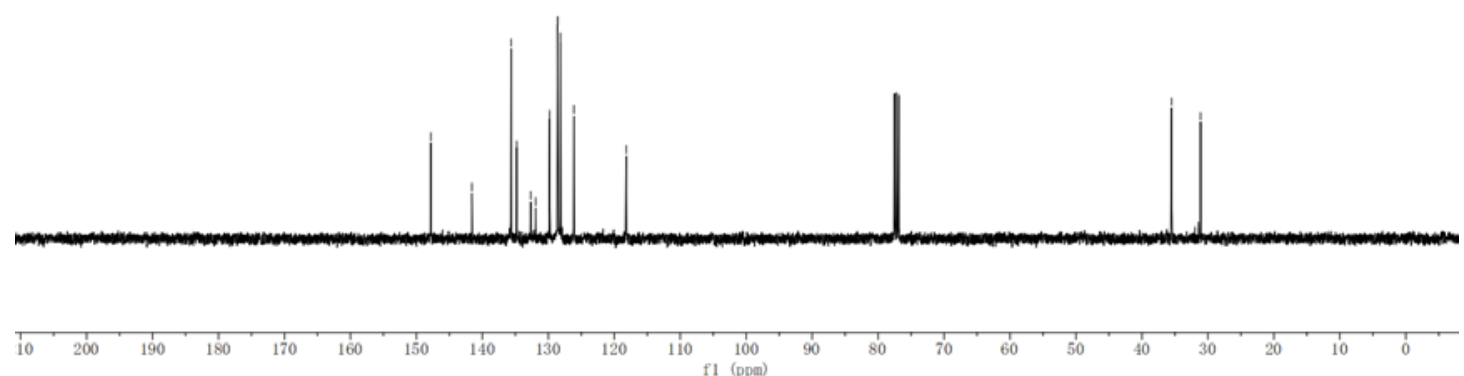

${ }^{13} \mathrm{C}$ NMR (101 MHz, $\mathrm{CDCl}_{3}$ ) spectrum of 3d 


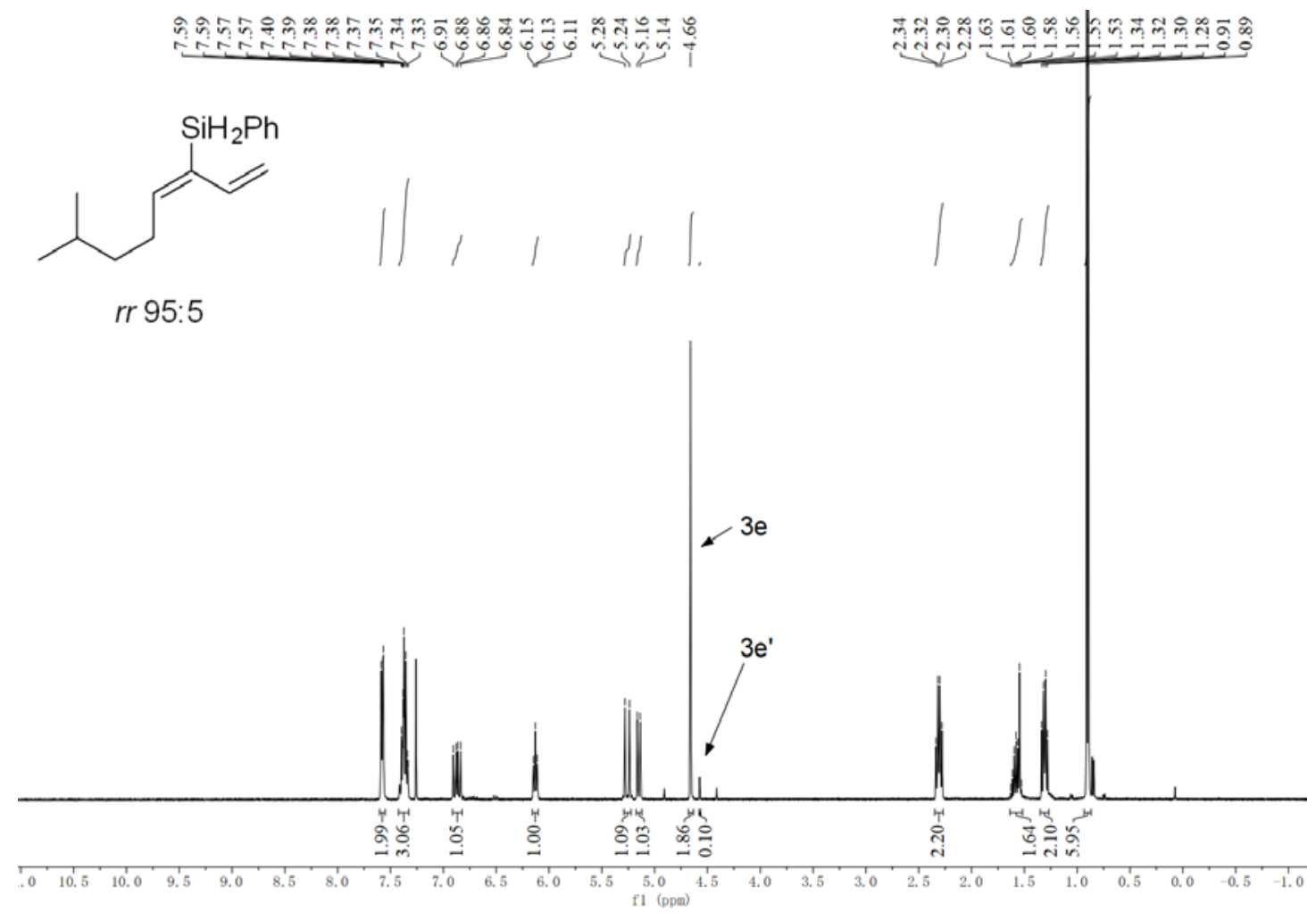

${ }^{1} \mathrm{H}$ NMR (400 MHz, $\mathrm{CDCl}_{3}$ ) spectrum of $\mathbf{3 e}$

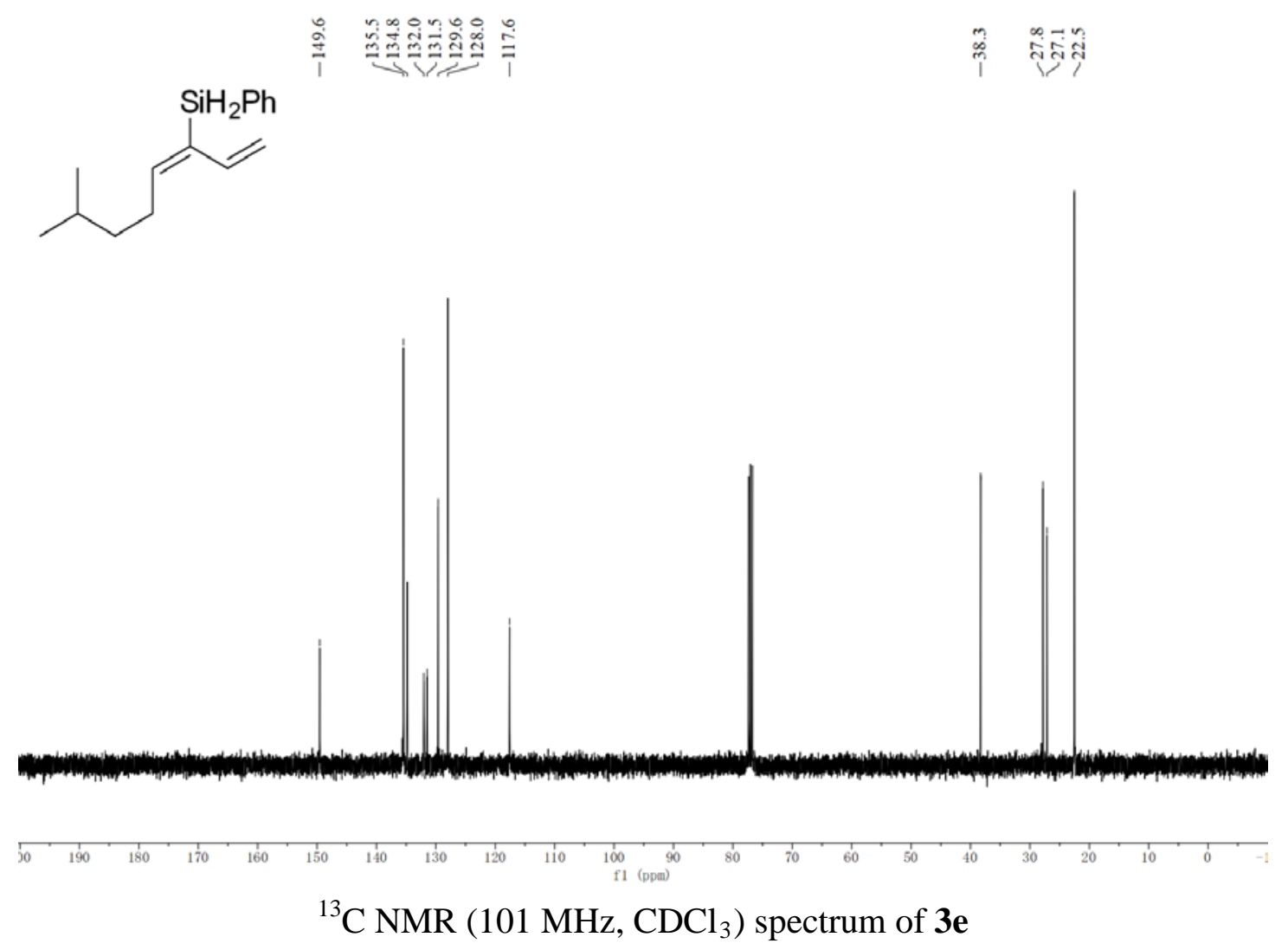


<smiles>C=C/C(=C\CC1CCCCC1)[SH2+]c1ccccc1</smiles>

rr 98:2
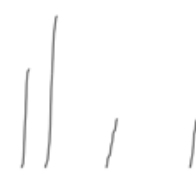

11

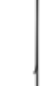

.

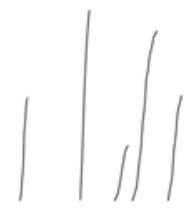

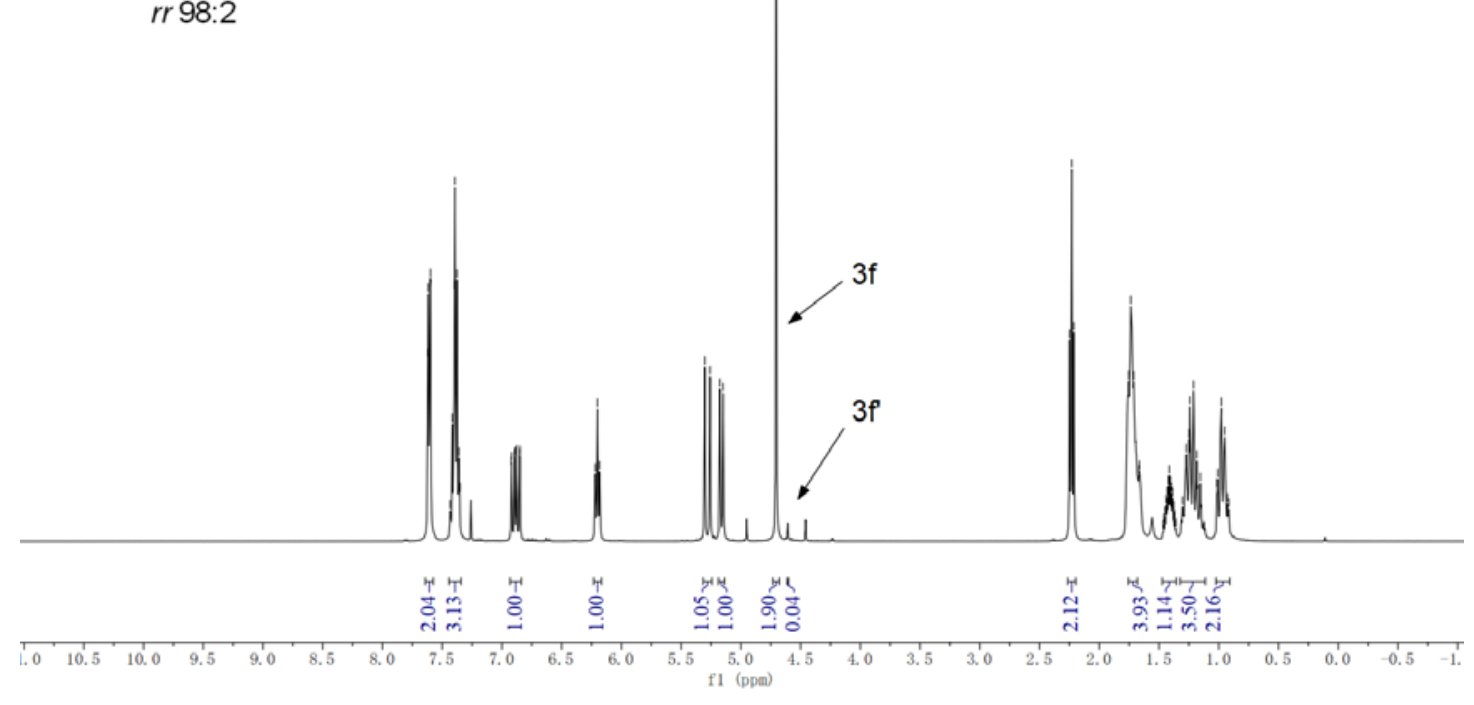

${ }^{1} \mathrm{H}$ NMR (400 MHz, $\left.\mathrm{CDCl}_{3}\right)$ spectrum of $\mathbf{3 f}$

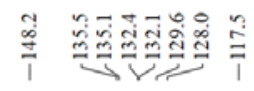<smiles>C=C/C(=C\CC1CCCCC1)[SH+]c1ccccc1</smiles>
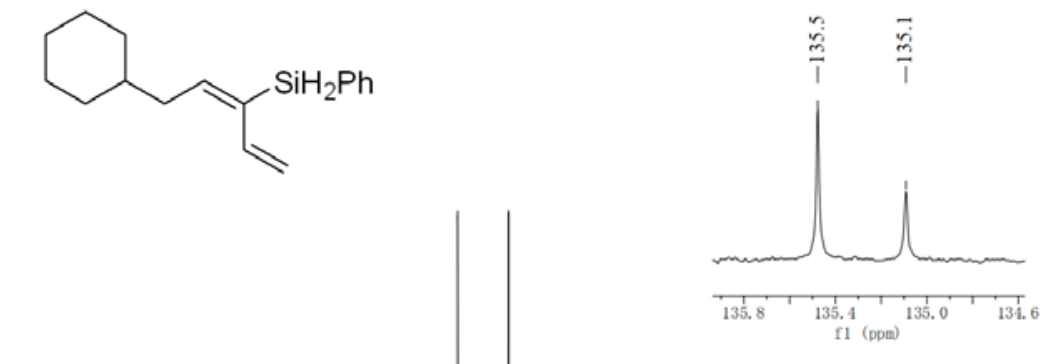

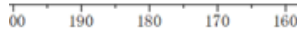

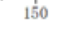

${ }^{13} \mathrm{C}$ NMR (101 MHz, $\mathrm{CDCl}_{3}$ ) spectrum of $\mathbf{3 f}$ 


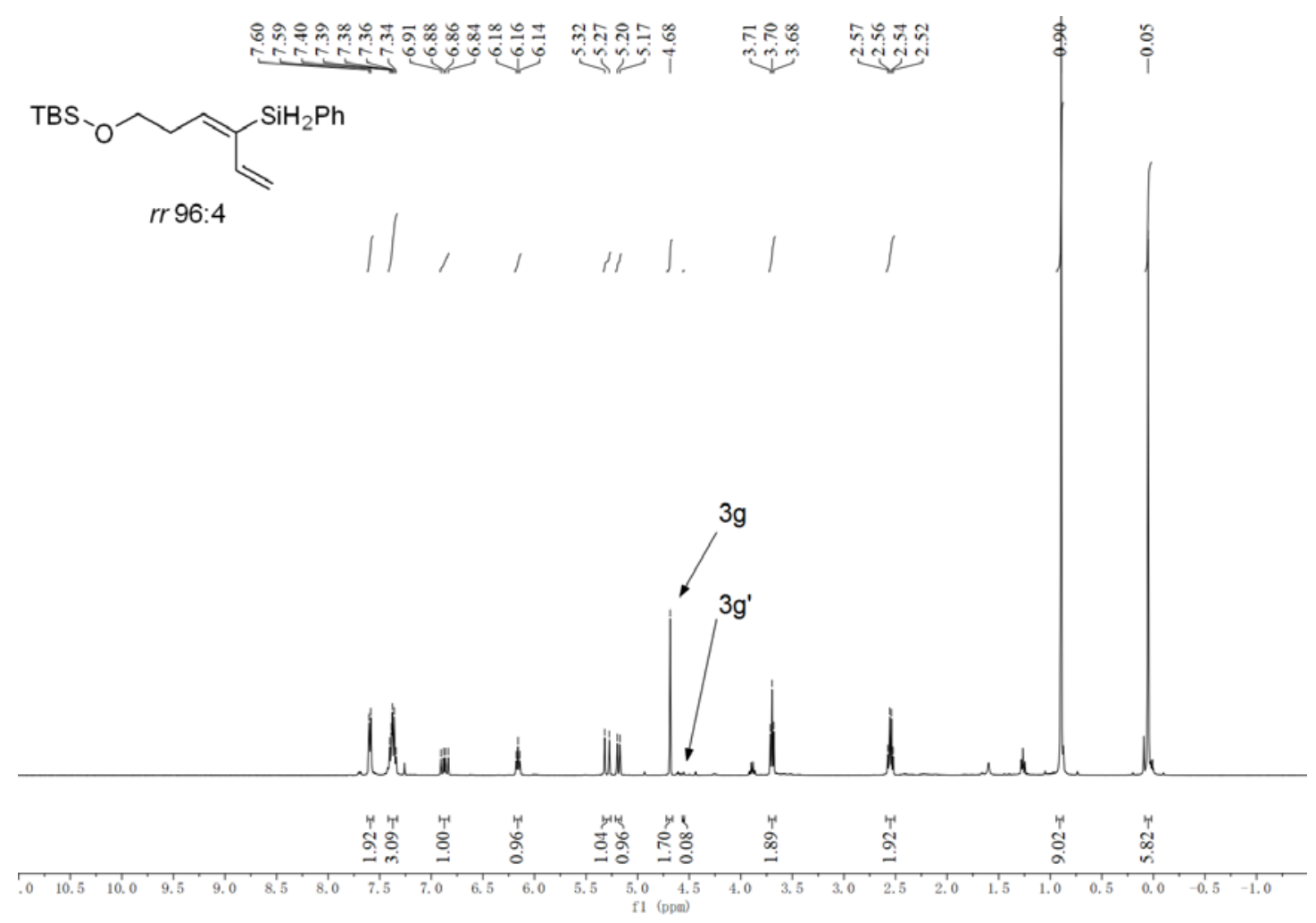

${ }^{1} \mathrm{H}$ NMR (400 MHz, $\mathrm{CDCl}_{3}$ ) spectrum of $\mathbf{3 g}$

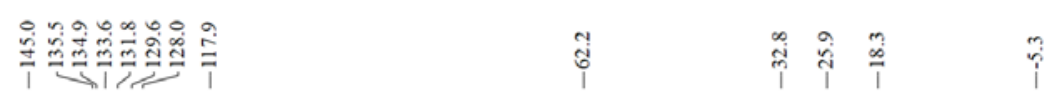

TBS $\mathrm{SiH}_{2} \mathrm{Ph}$
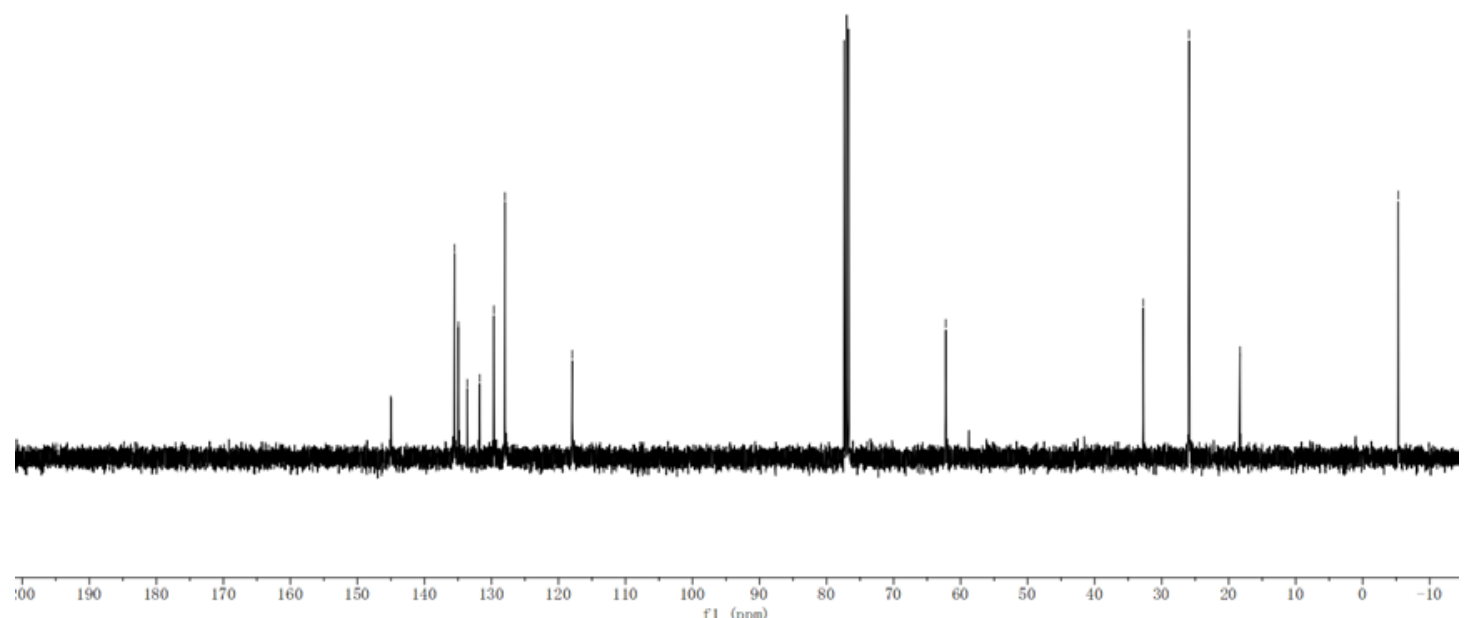

${ }^{13} \mathrm{C}$ NMR (101 MHz, $\mathrm{CDCl}_{3}$ ) spectrum of $\mathbf{3 g}$ 


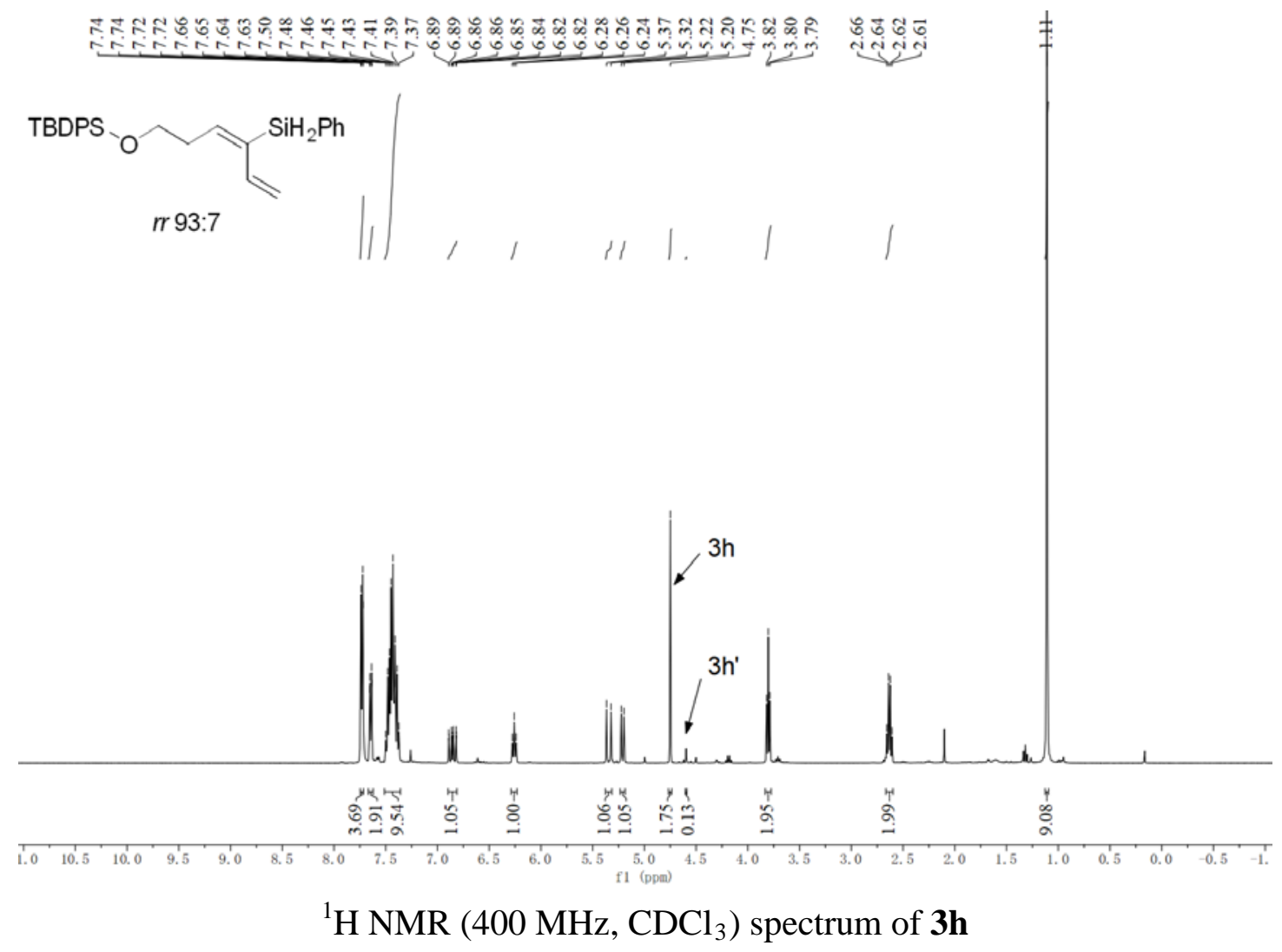

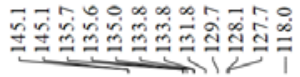

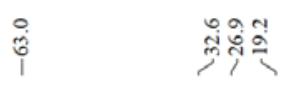

TBDPS、次 $\mathrm{SiH}_{2} \mathrm{Ph}$

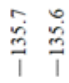
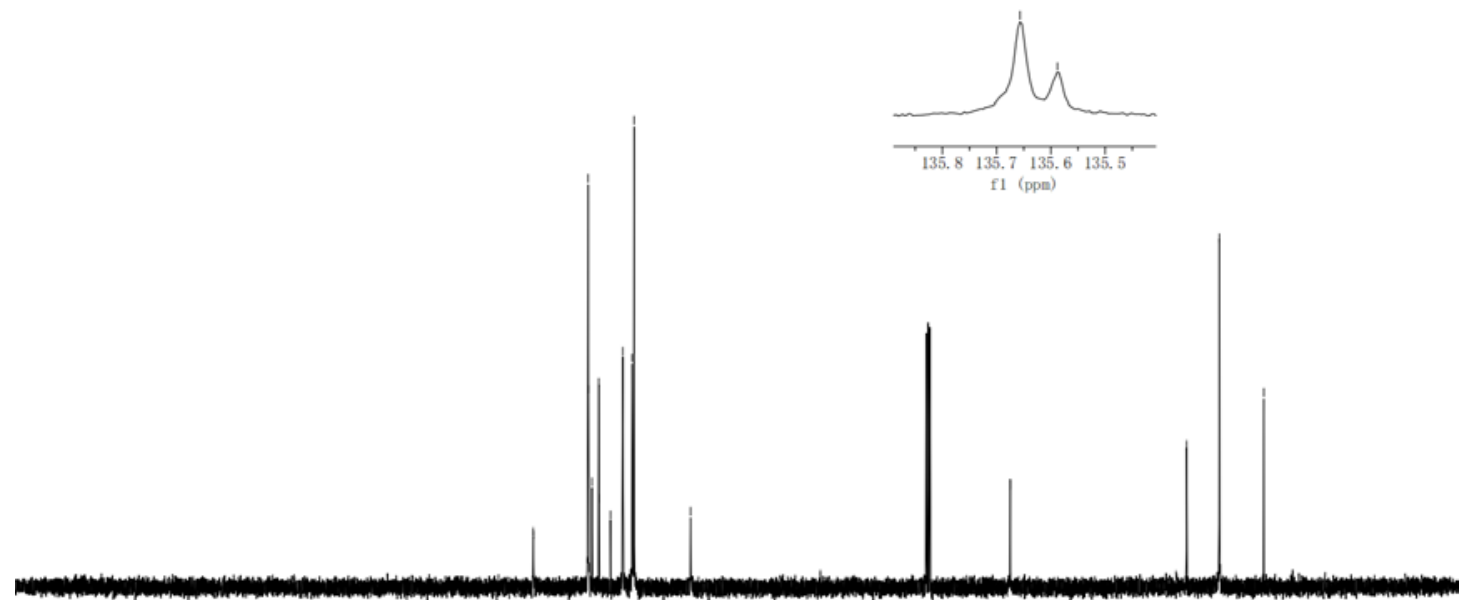

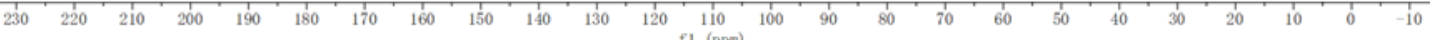

${ }^{13} \mathrm{C}$ NMR (101 MHz, $\mathrm{CDCl}_{3}$ ) spectrum of $\mathbf{3 h}$ 

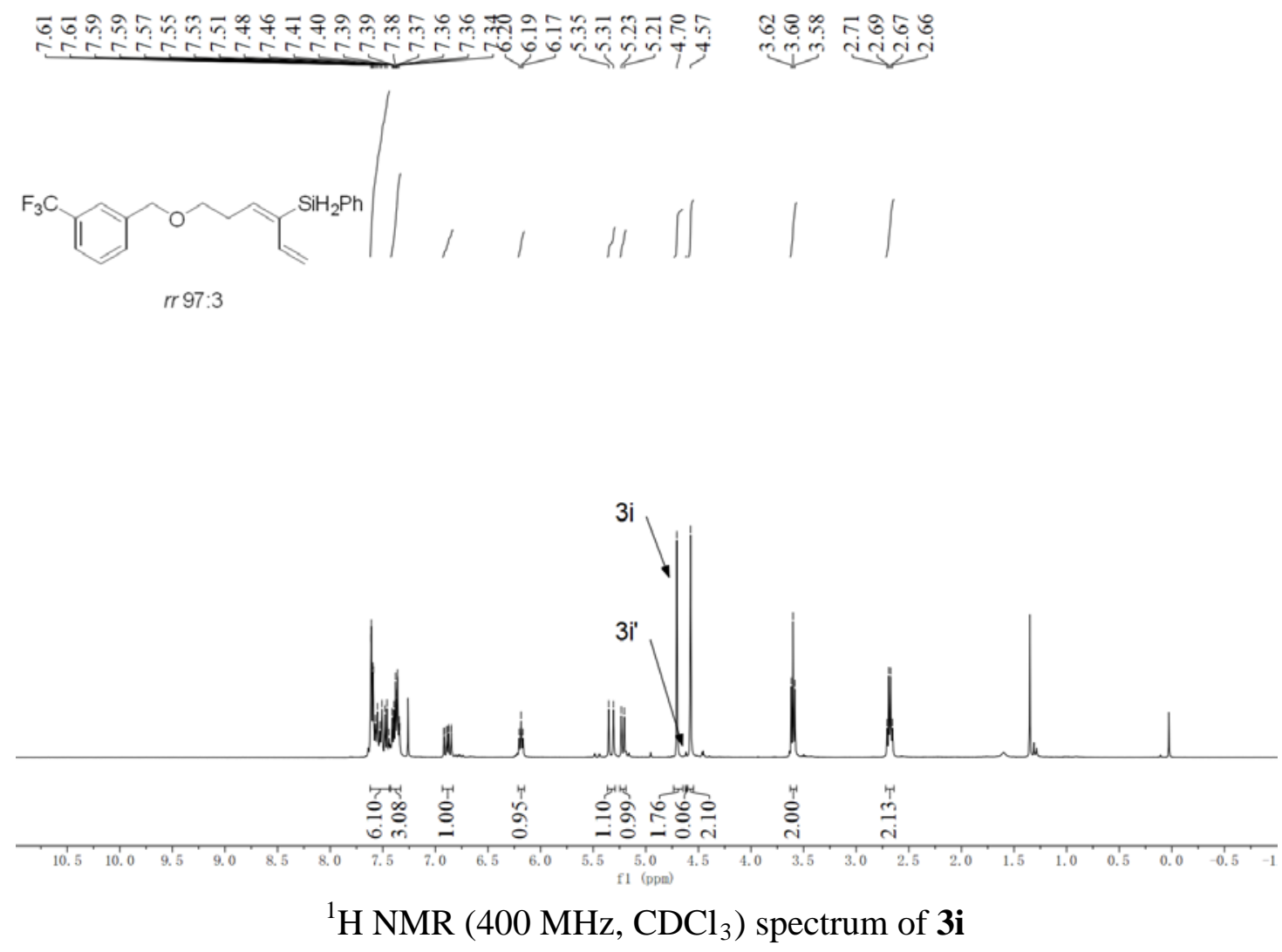

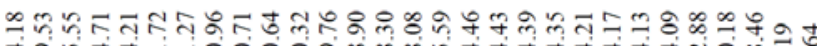

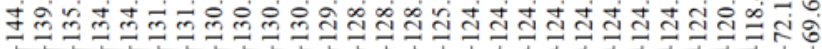

ลิ่<smiles>C=C/C(=C\CCOCc1cccc(C(F)(F)F)c1)[SH+](C=C)(/C=C\c1ccccc1)C(F)(F)F</smiles>

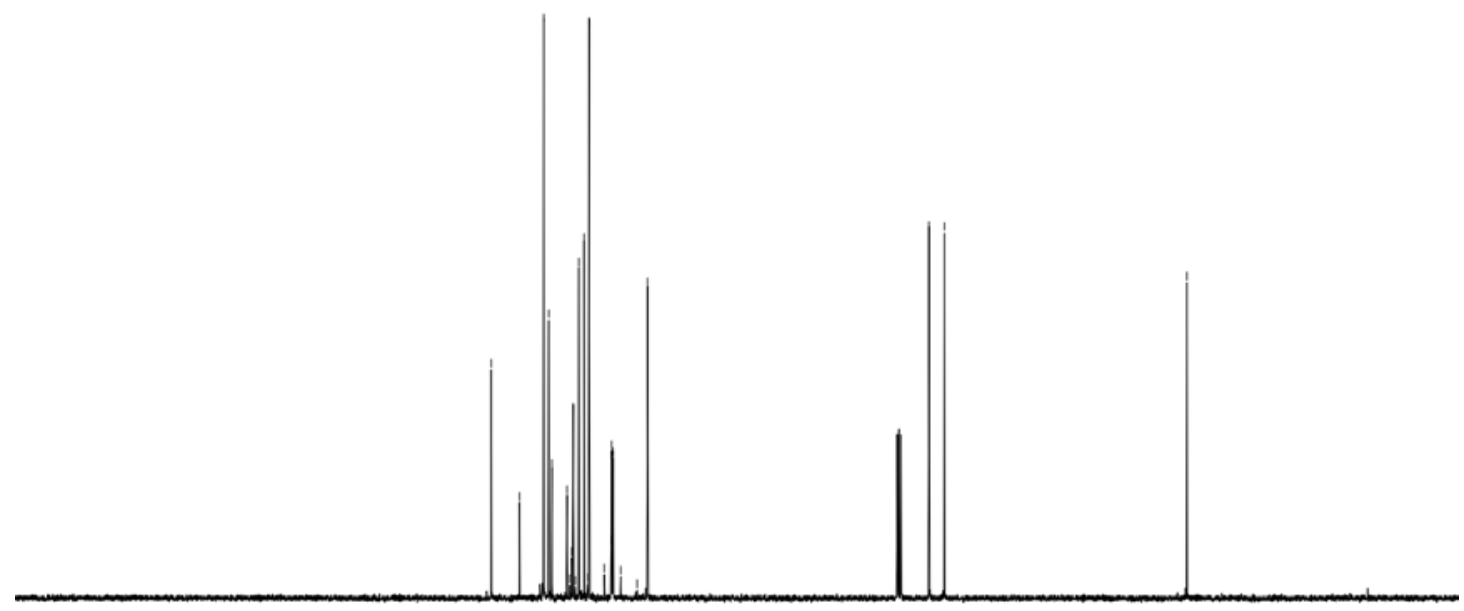

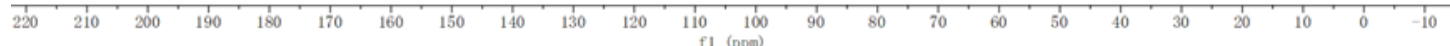

${ }^{13} \mathrm{C}$ NMR (101 MHz, $\mathrm{CDCl}_{3}$ ) spectrum of $3 \mathbf{i}$ 
$\mathrm{F}_{3} \mathrm{C}$
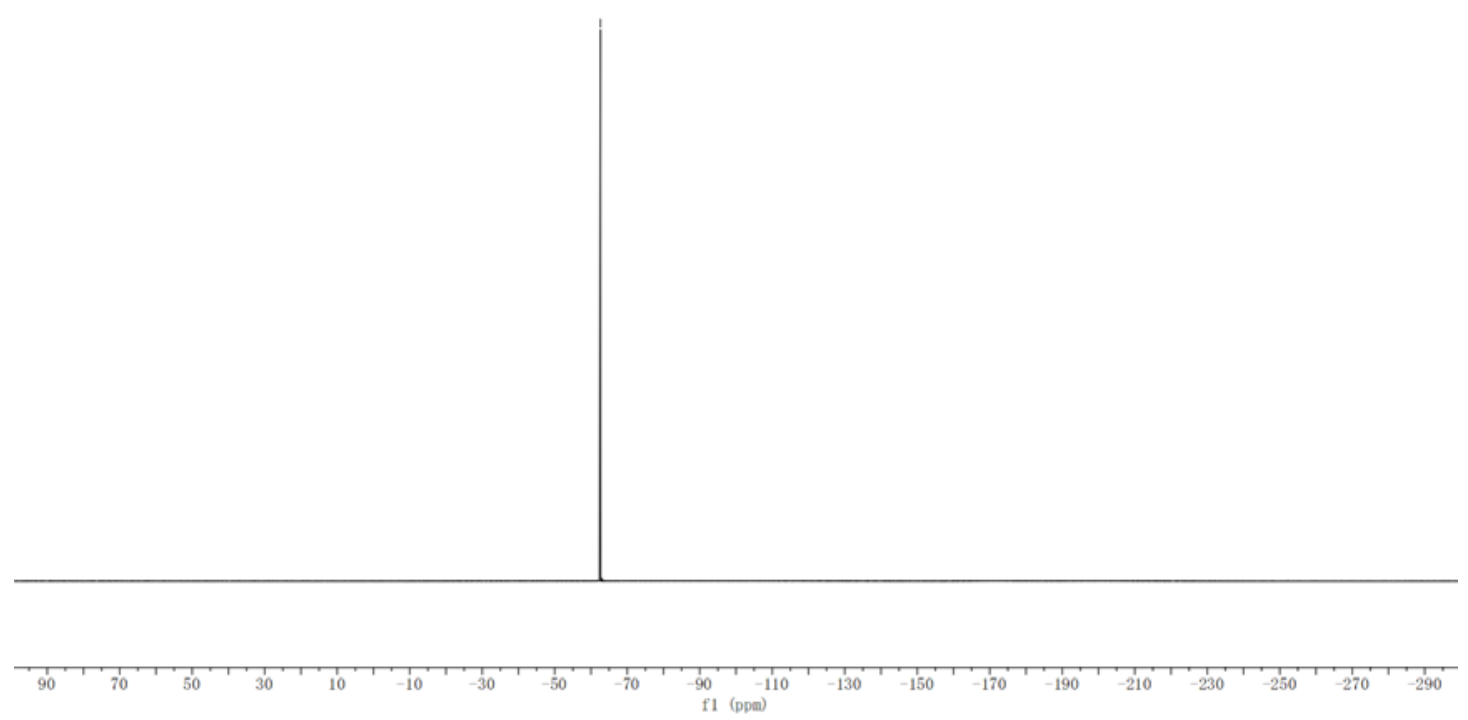

${ }^{19} \mathrm{~F} \mathrm{NMR}\left(376 \mathrm{MHz}, \mathrm{CDCl}_{3}\right.$ ) spectrum of $\mathbf{3 i}$
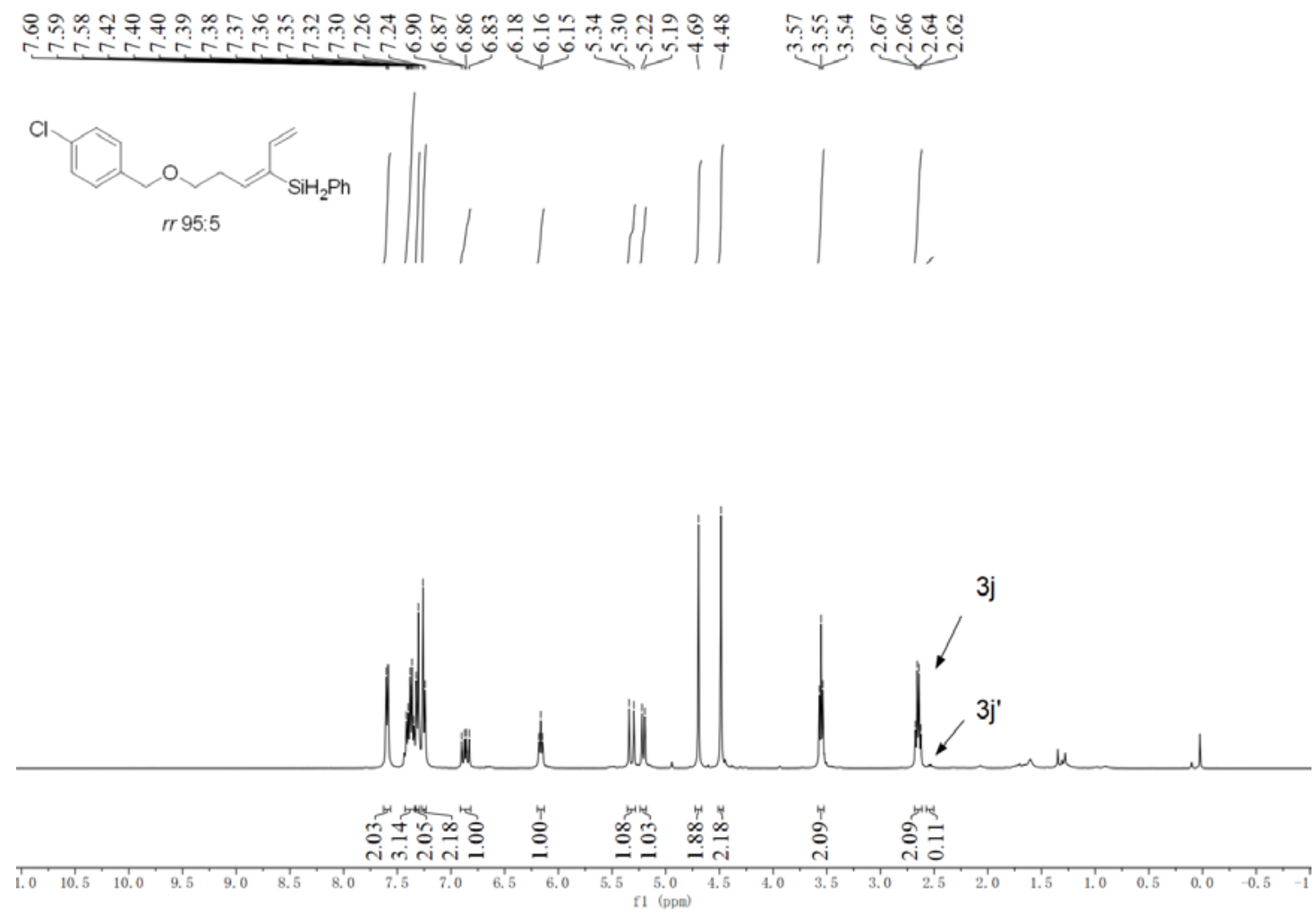

${ }^{1} \mathrm{H}$ NMR (400 MHz, $\mathrm{CDCl}_{3}$ ) spectrum of $\mathbf{3 j}$ 
<smiles>C=C/C(=C\CCOCc1ccc(Cl)cc1)Sc1ccccc1</smiles>
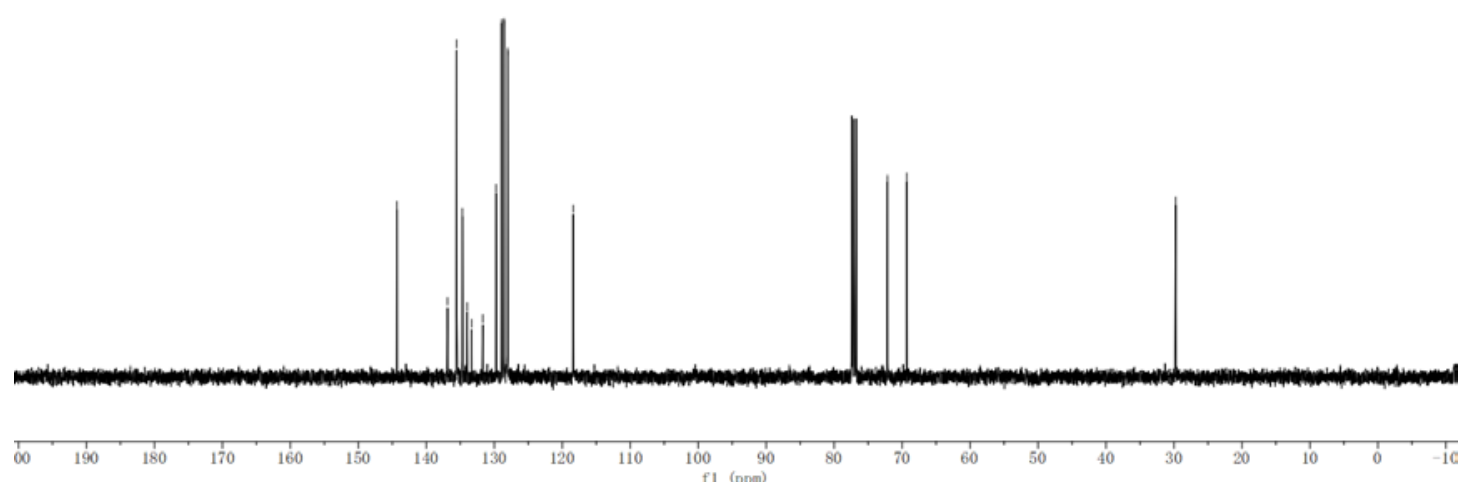

${ }^{13} \mathrm{C}$ NMR (101 MHz, $\left.\mathrm{CDCl}_{3}\right)$ spectrum of $\mathbf{3 j}$
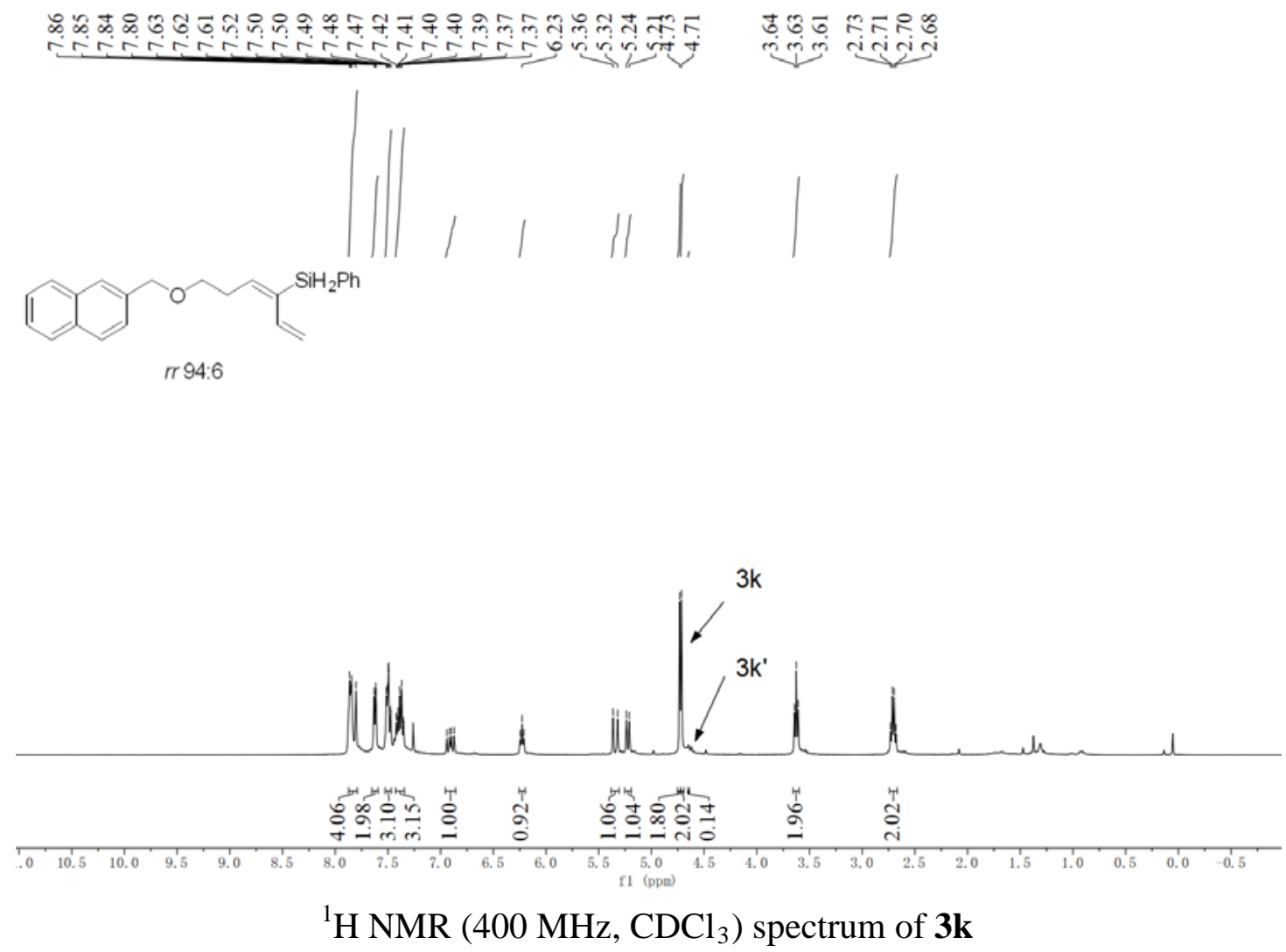
<smiles>C=C/C(=C\CCOCc1ccc2ccccc2c1)Sc1ccccc1</smiles>
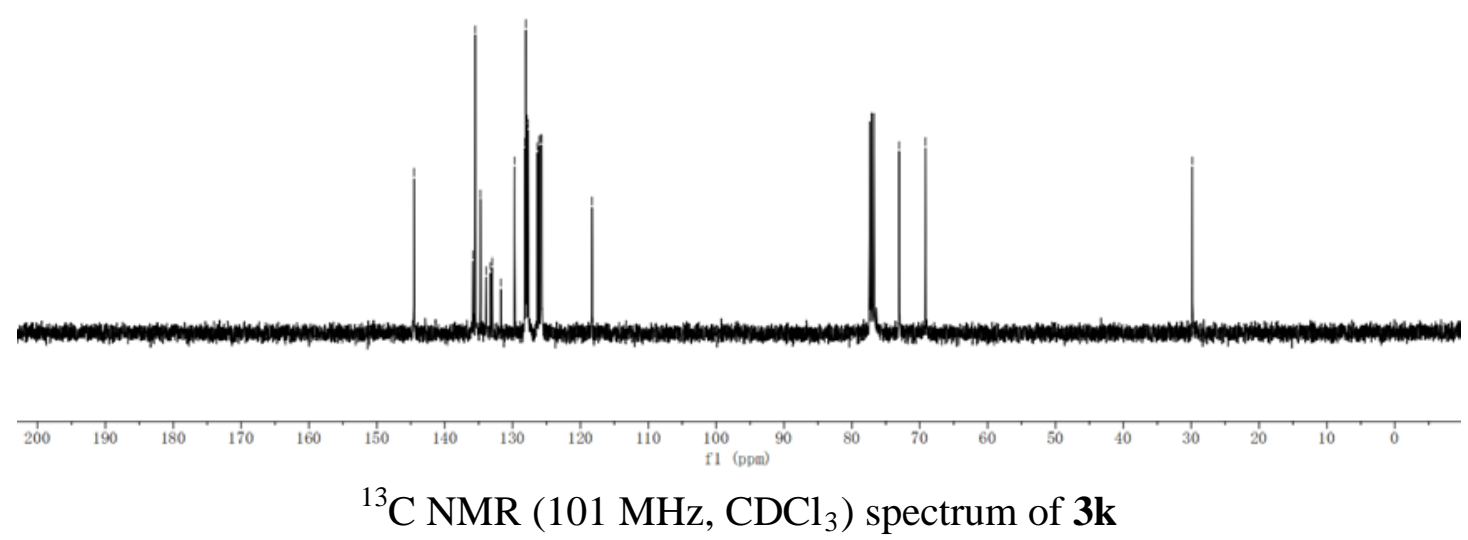

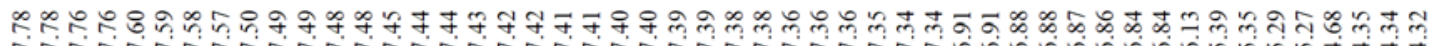

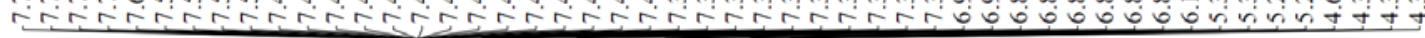<smiles>C#CC(C)C</smiles>

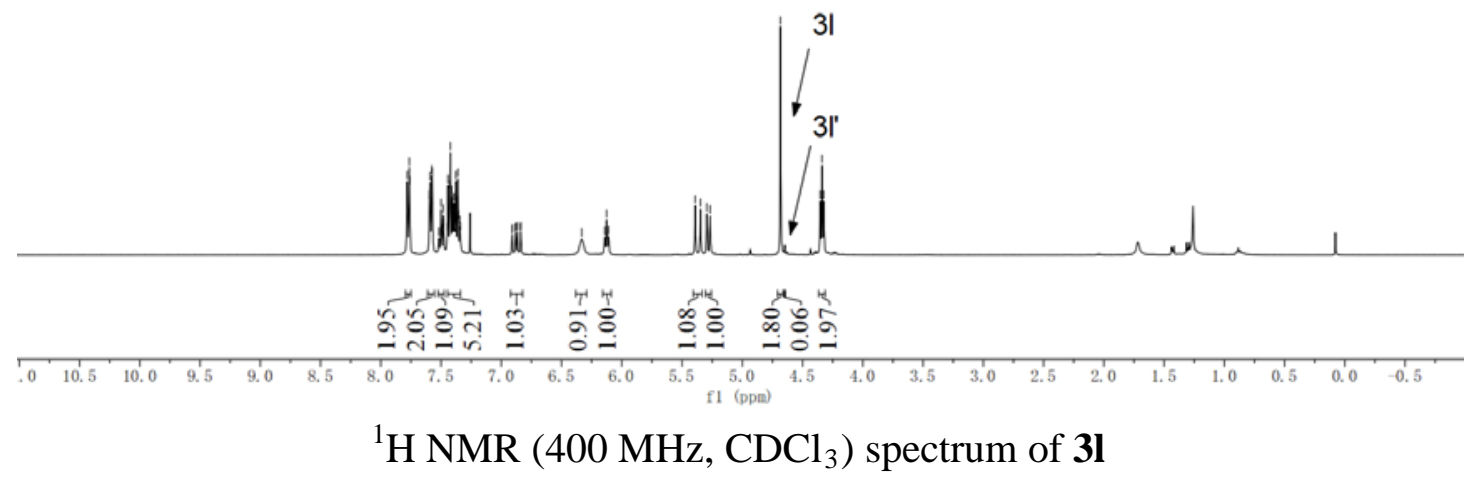



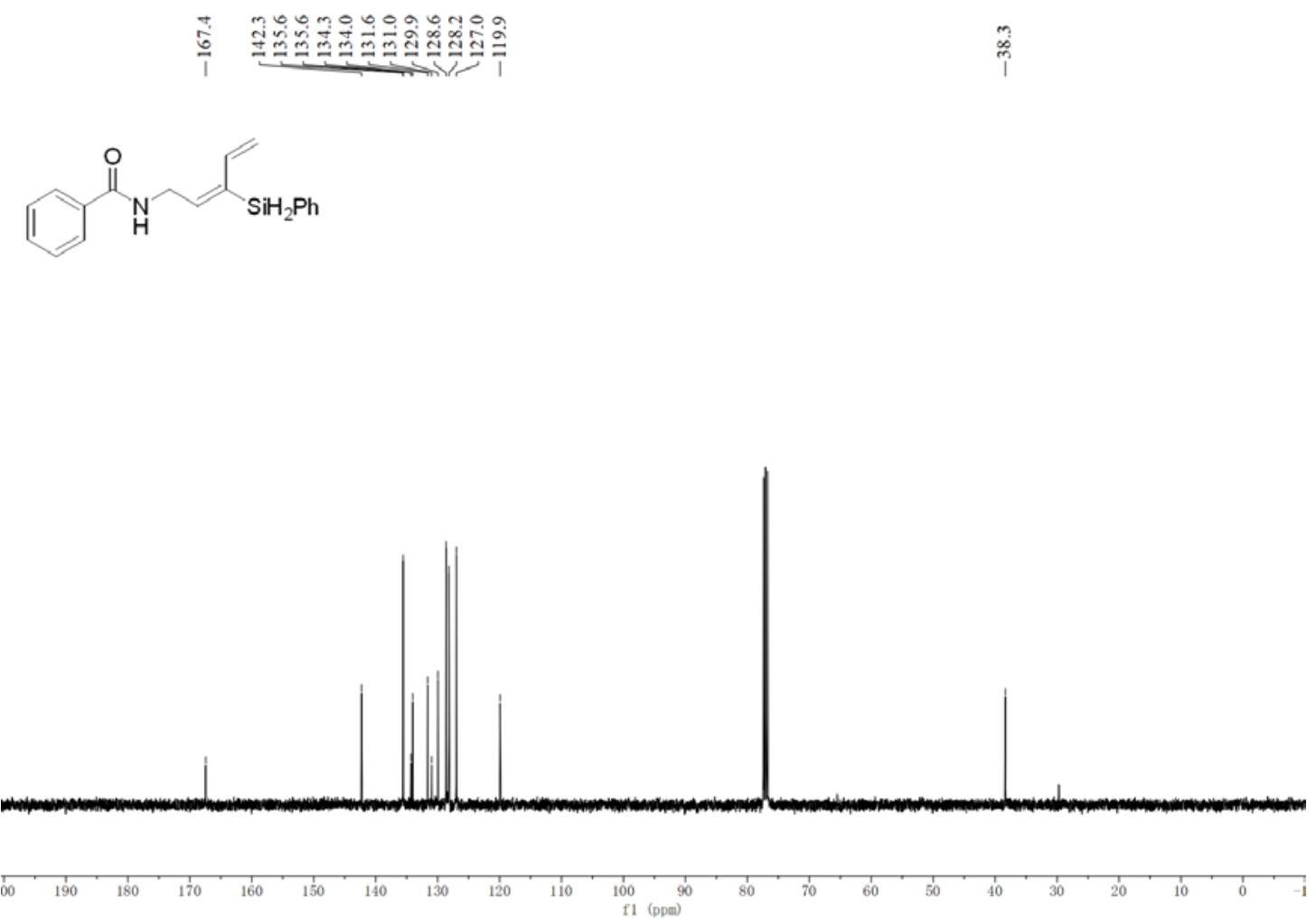

${ }^{13} \mathrm{C}$ NMR (101 MHz, $\mathrm{CDCl}_{3}$ ) spectrum of $3 \mathbf{l}$
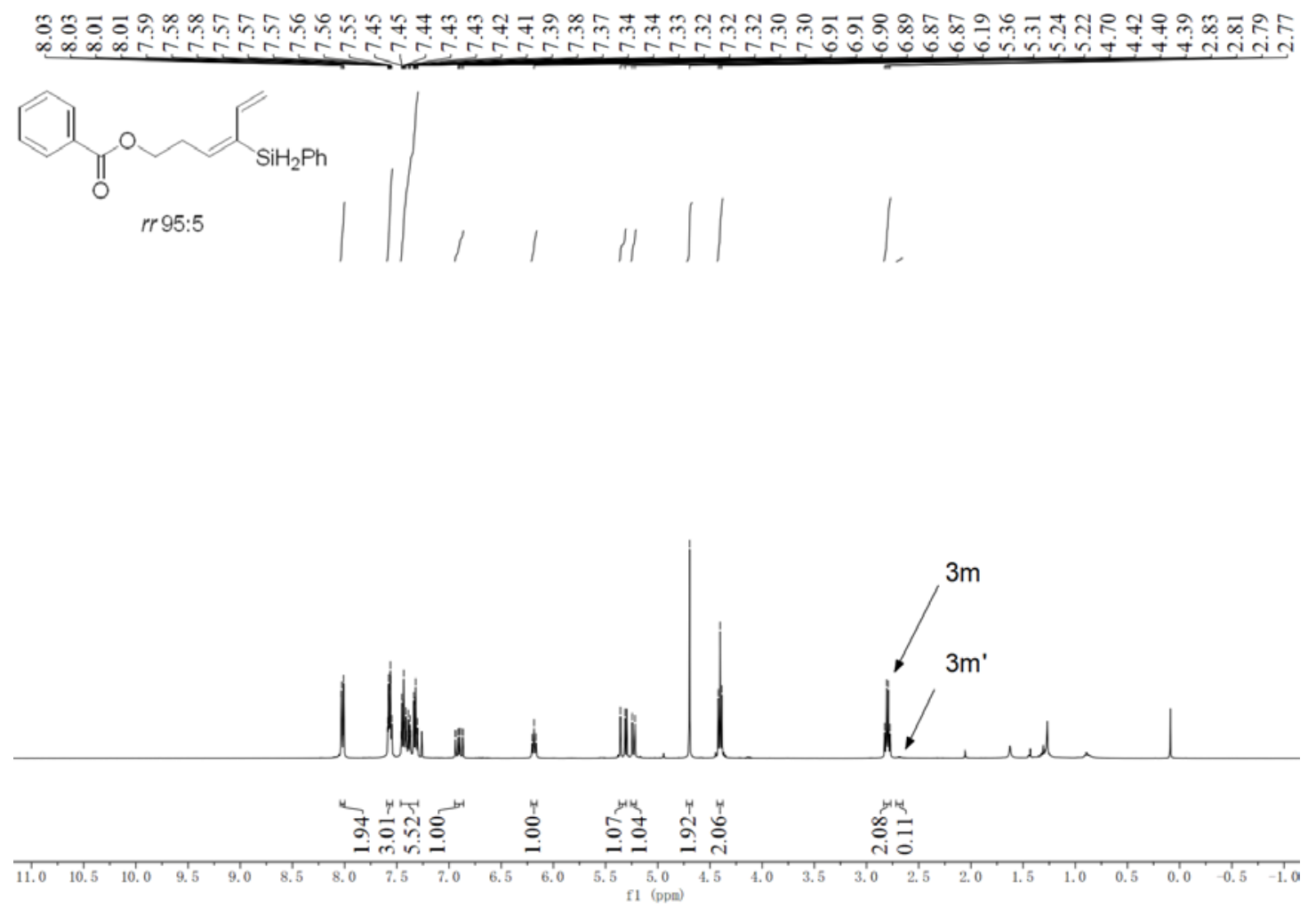

${ }^{1} \mathrm{H}$ NMR (400 MHz, $\mathrm{CDCl}_{3}$ ) spectrum of 3m 

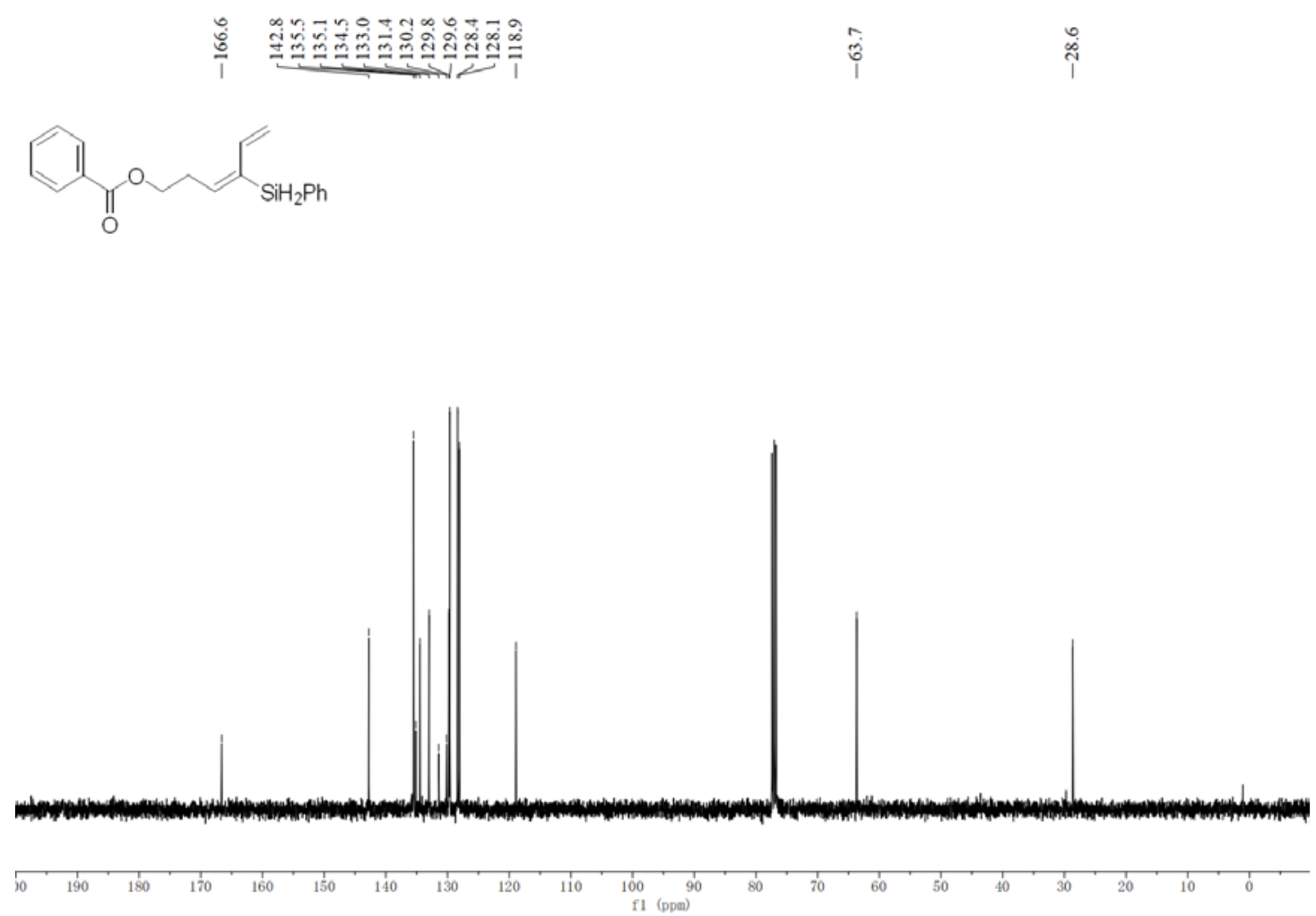

${ }^{13} \mathrm{C}$ NMR (101 MHz, $\mathrm{CDCl}_{3}$ ) spectrum of 3m
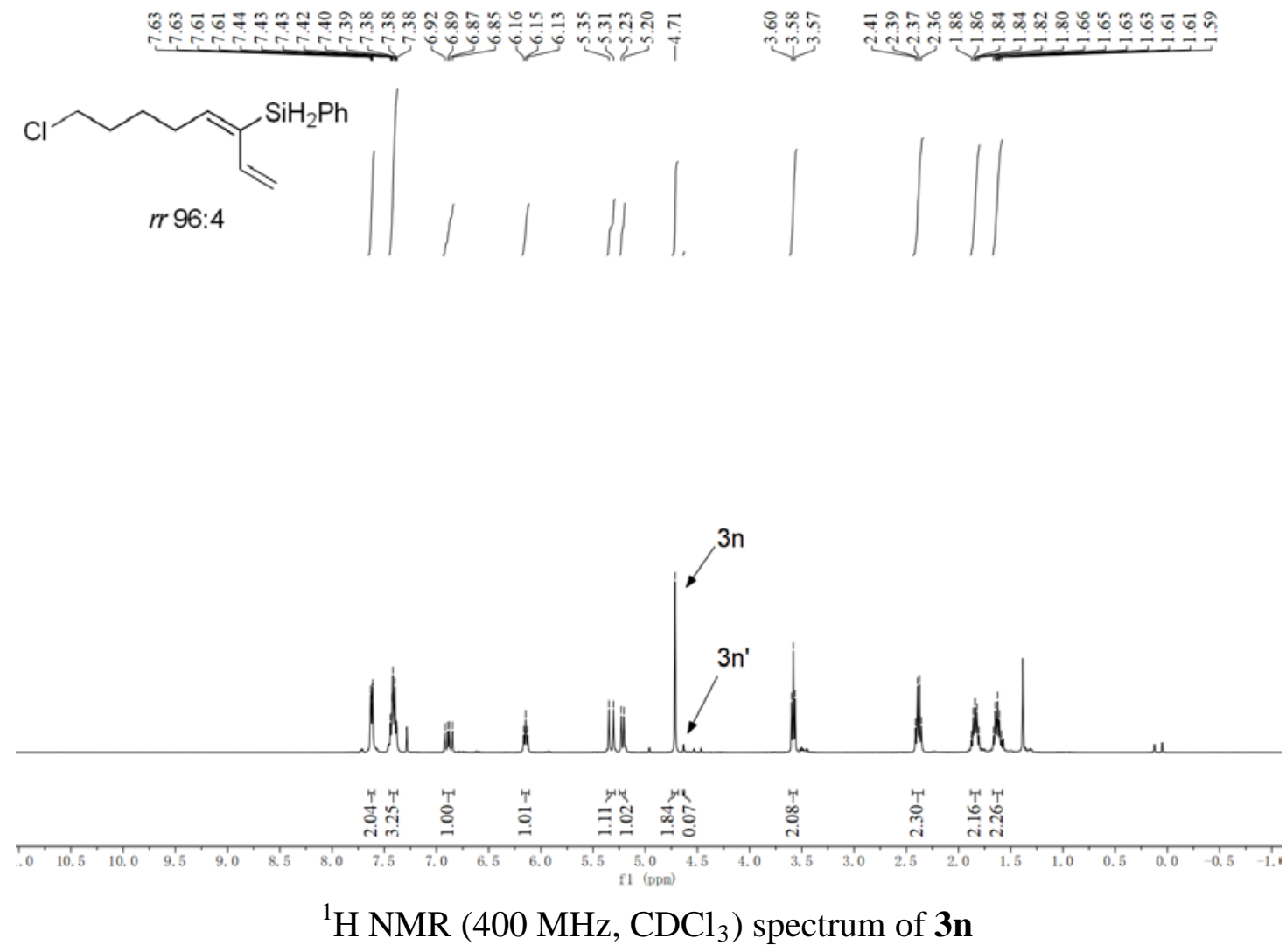
$\curvearrowright \mathrm{SiH}_{2} \mathrm{Ph}$
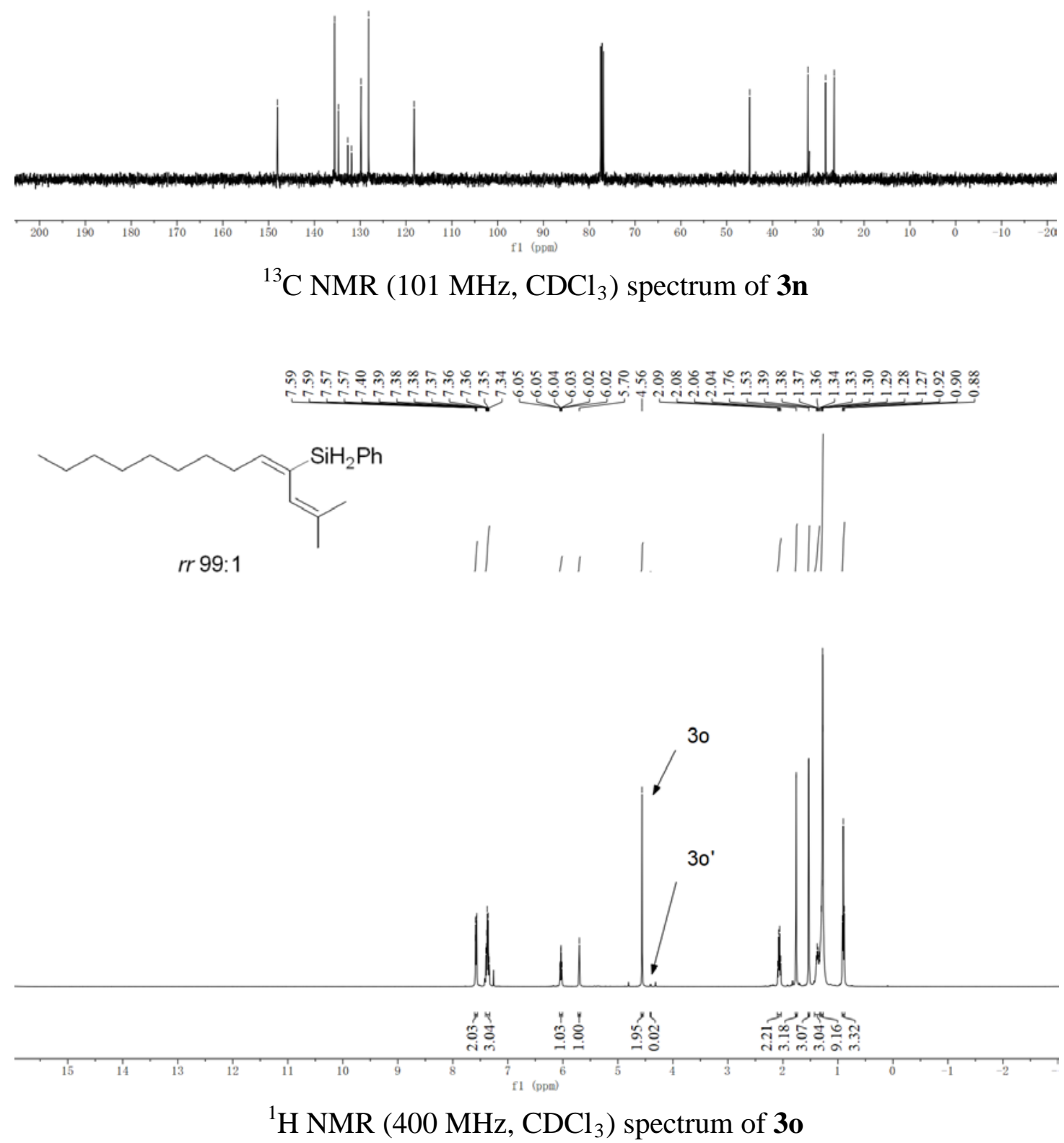

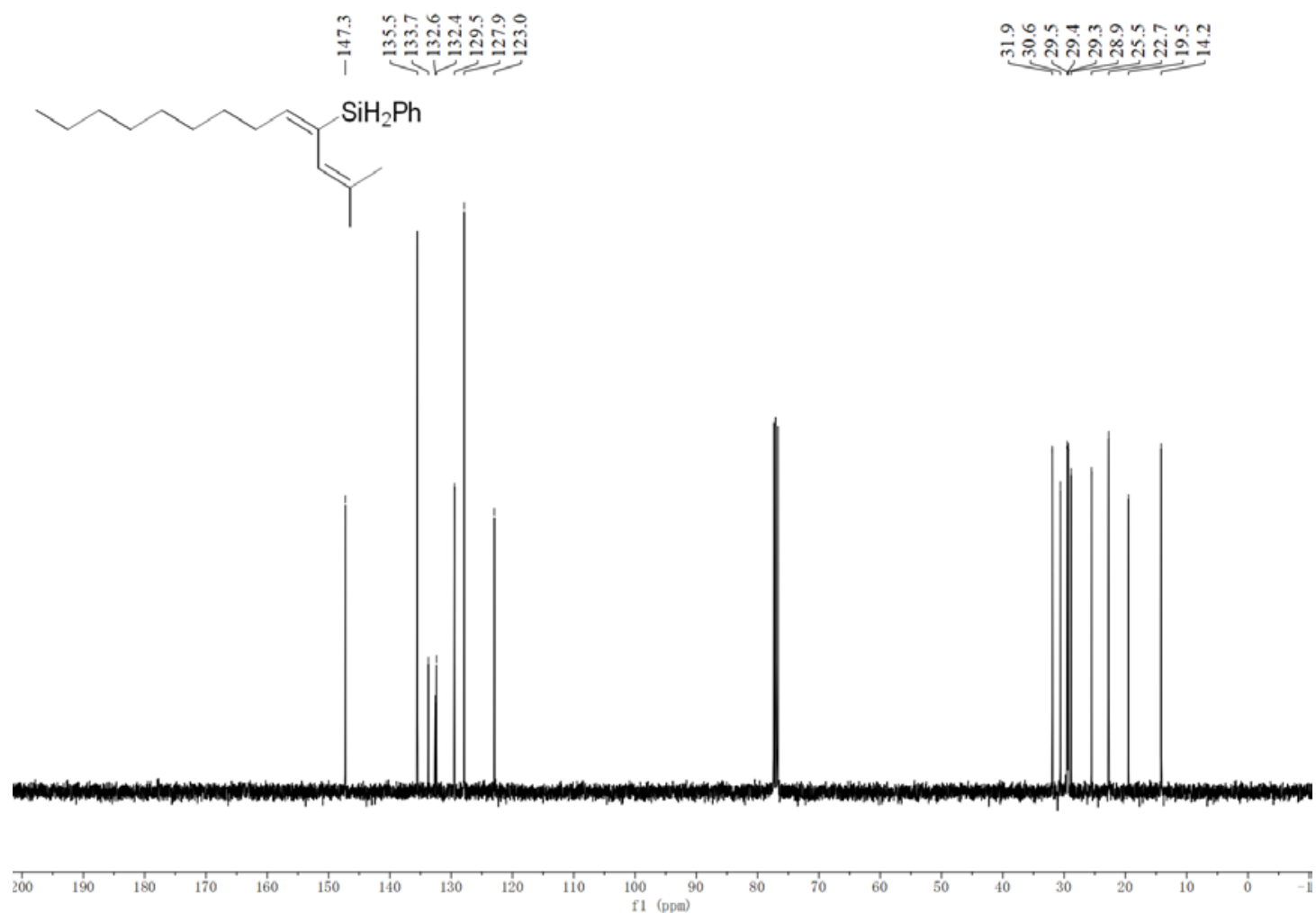

${ }^{13} \mathrm{C}$ NMR (101 MHz, $\left.\mathrm{CDCl}_{3}\right)$ spectrum of $3 \mathbf{0}$
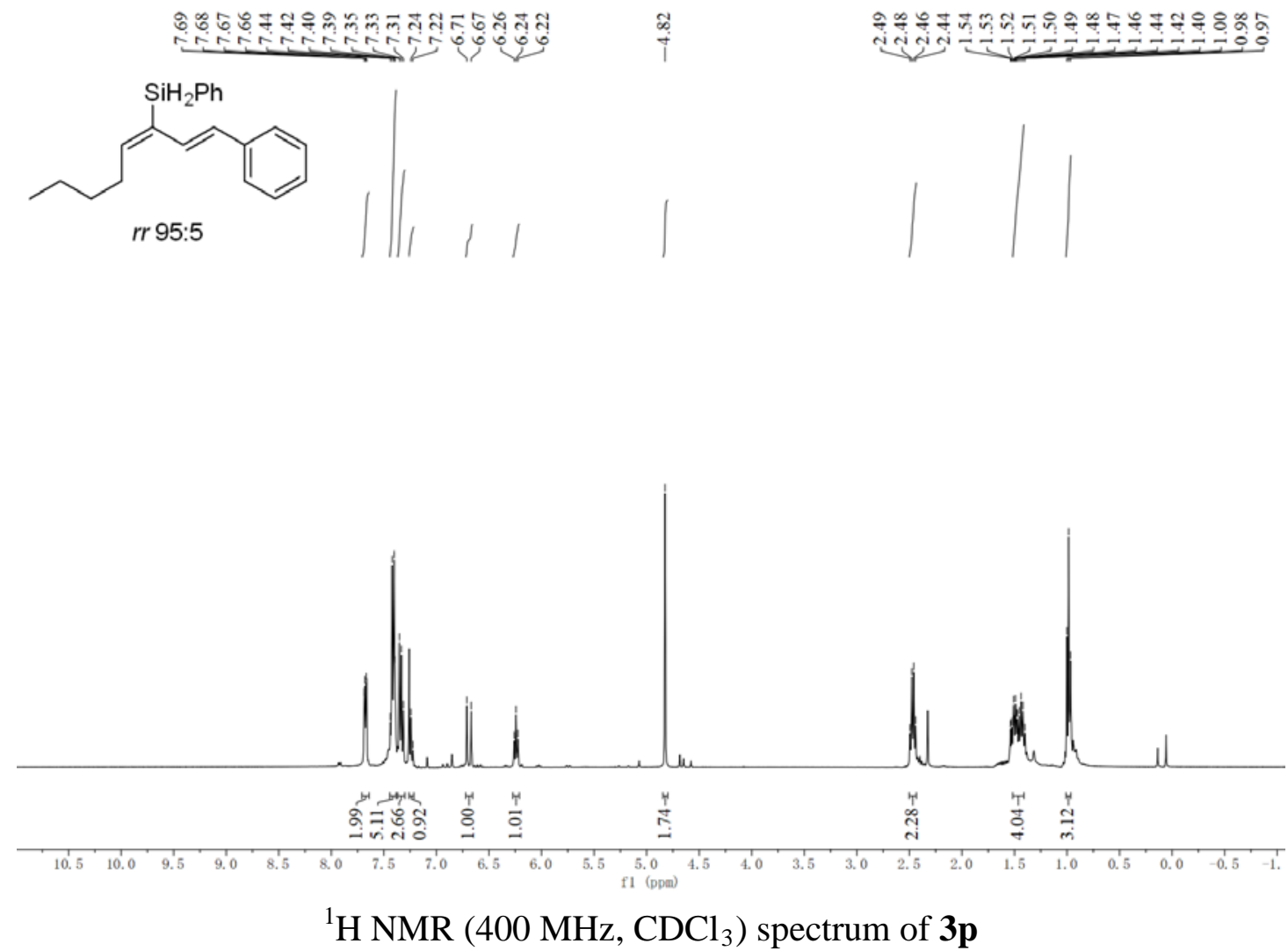

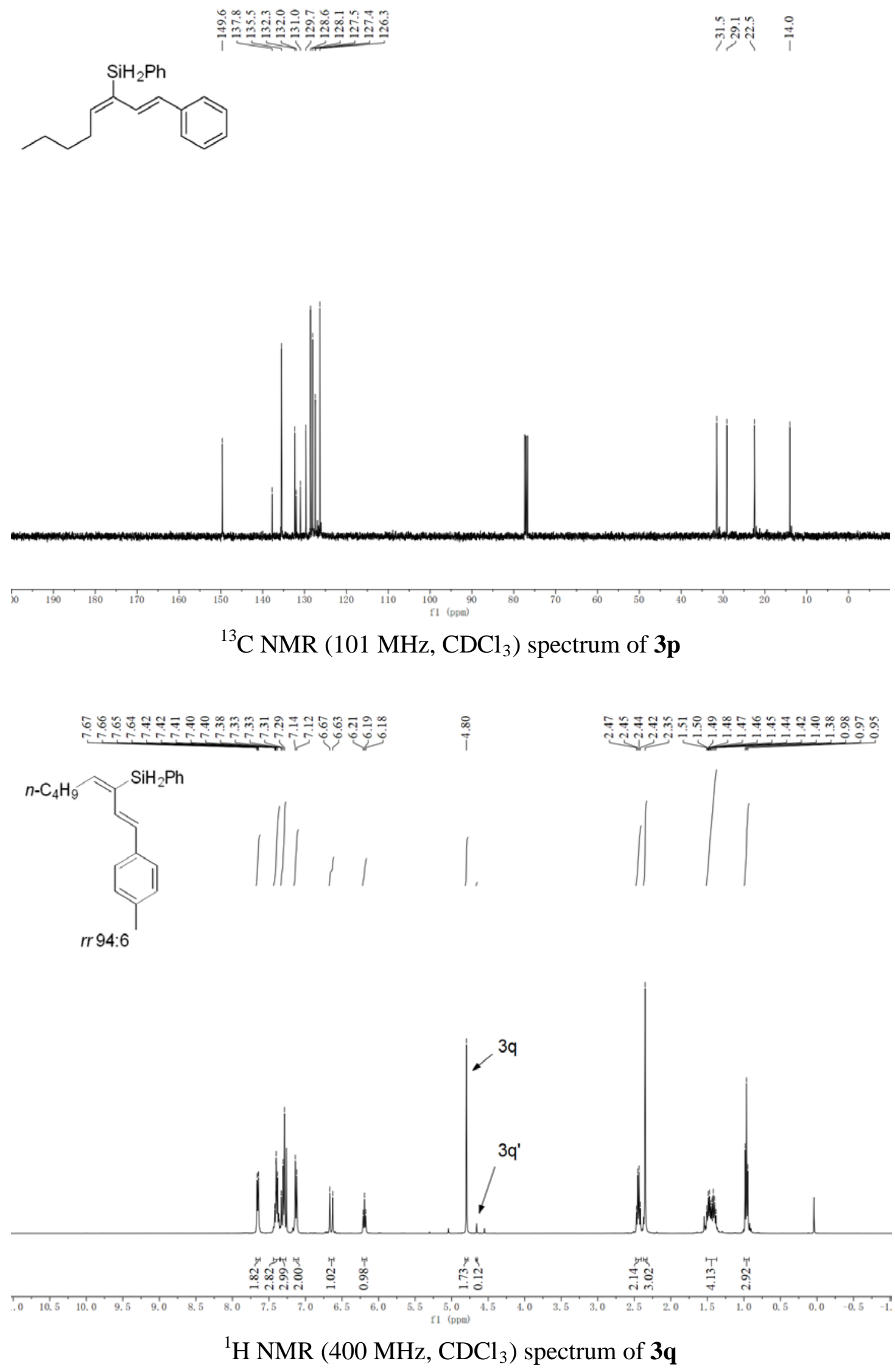

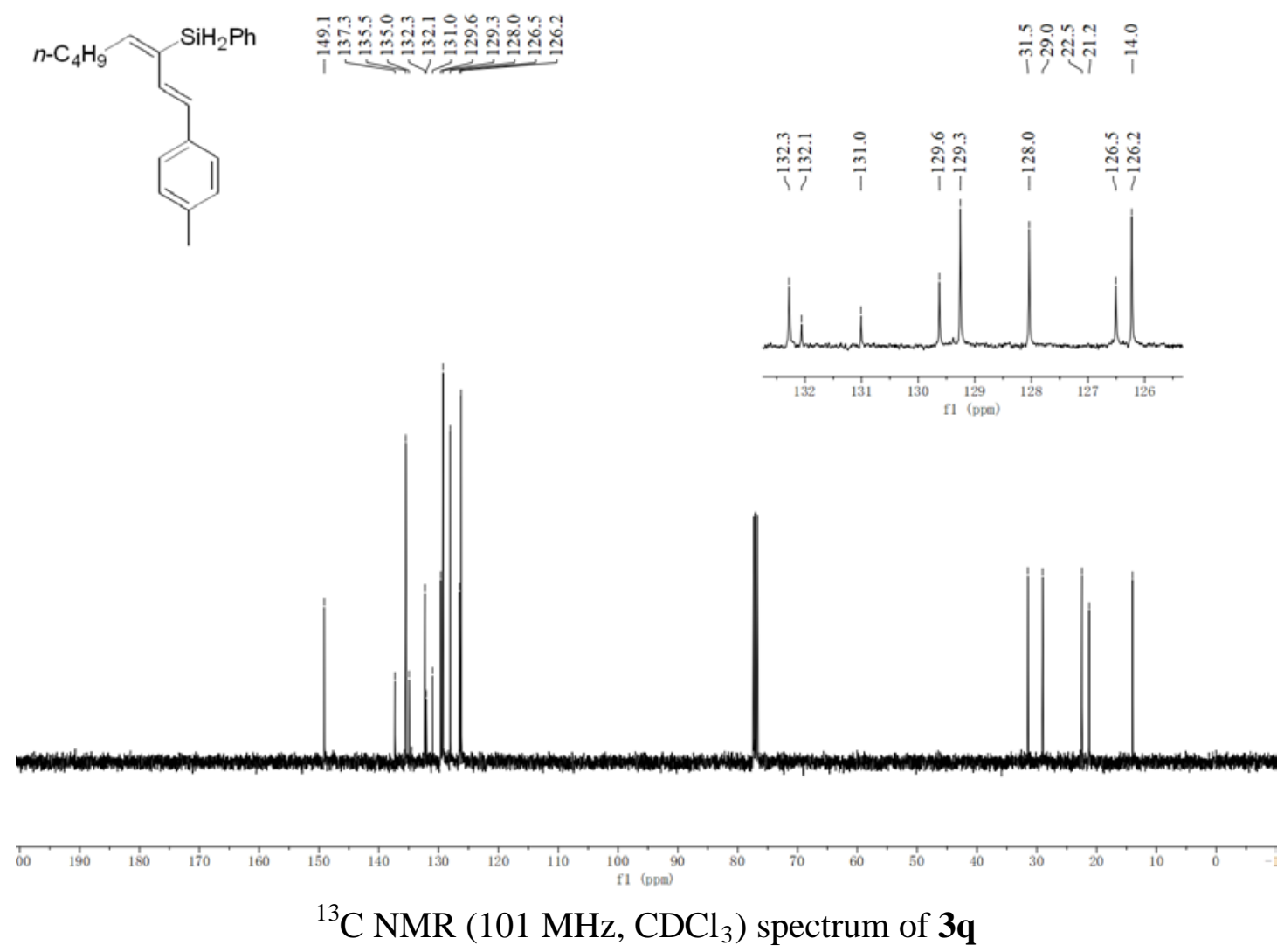

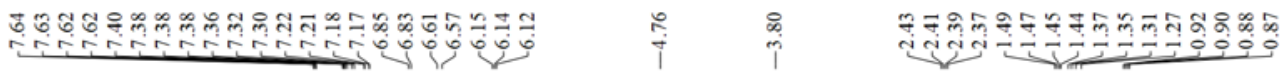
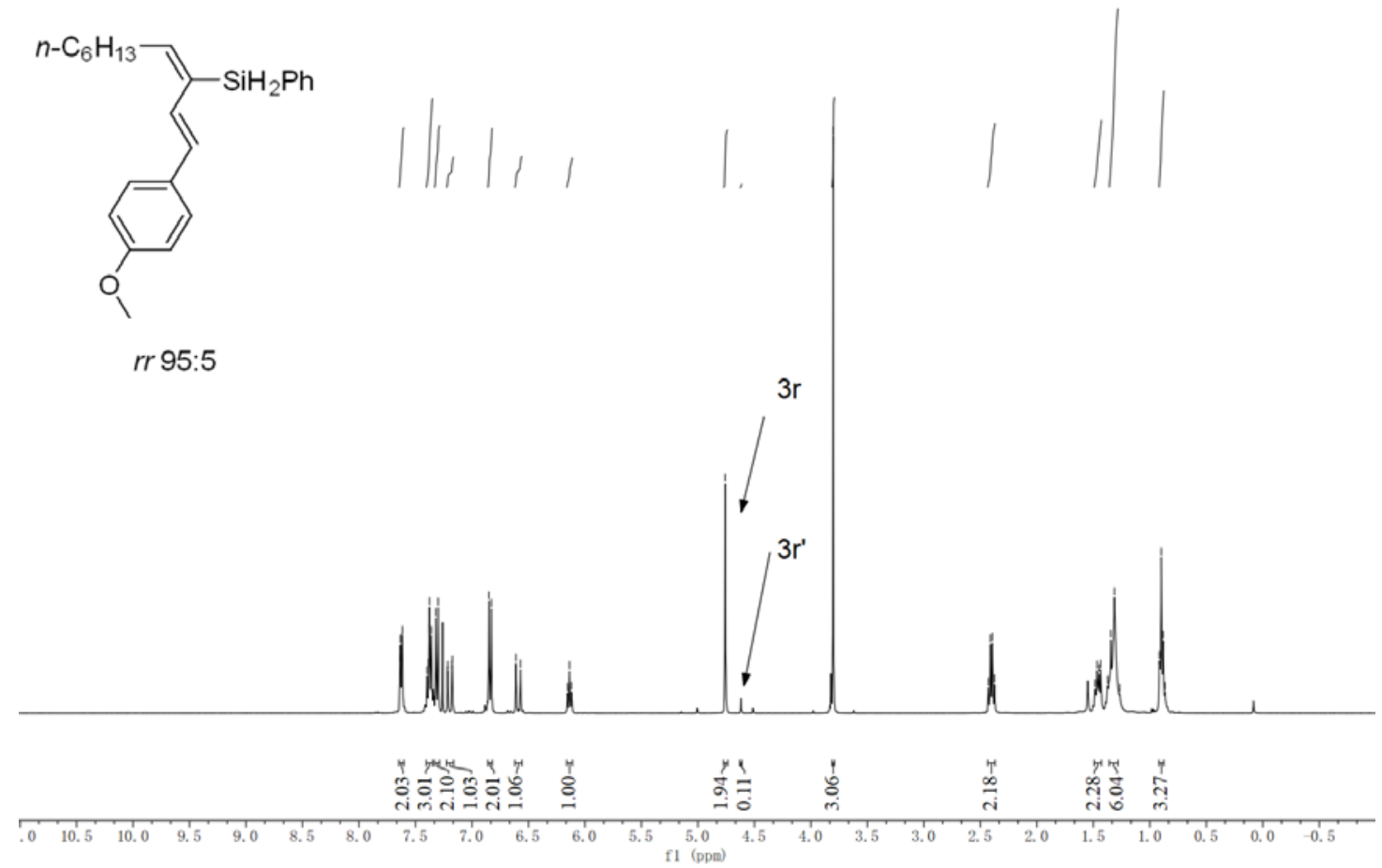

${ }^{1} \mathrm{H}$ NMR (400 MHz, $\mathrm{CDCl}_{3}$ ) spectrum of $3 \mathbf{r}$ 


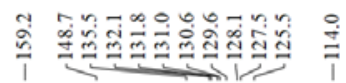

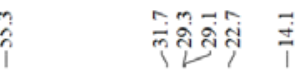

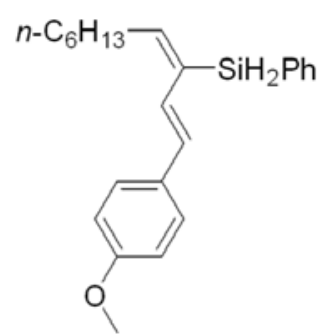

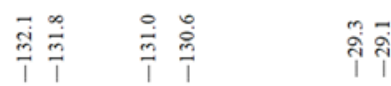
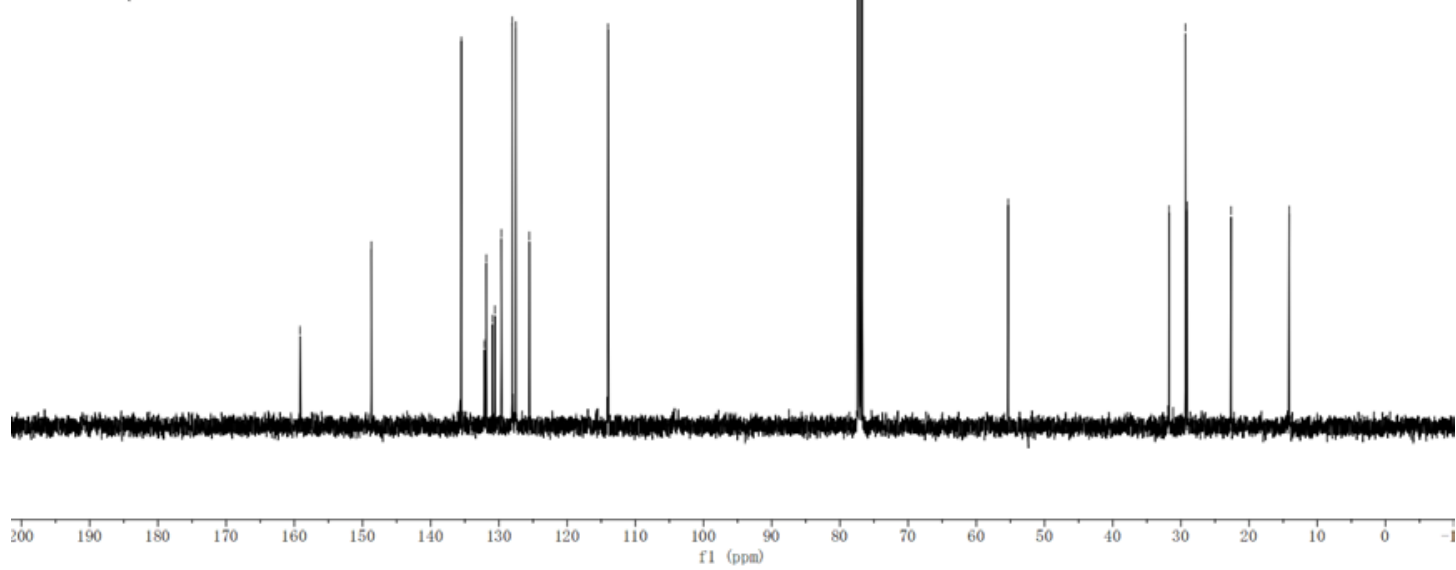

${ }^{13} \mathrm{C}$ NMR $\left(101 \mathrm{MHz}, \mathrm{CDCl}_{3}\right)$ spectrum of $\mathbf{3 r}$

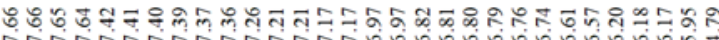

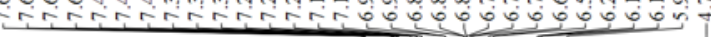

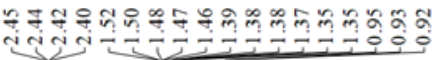
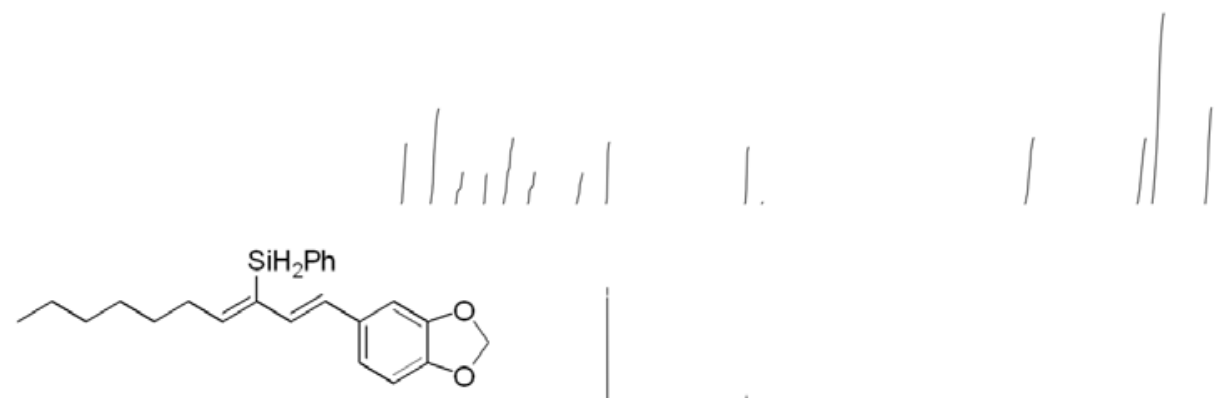

rr 95:5
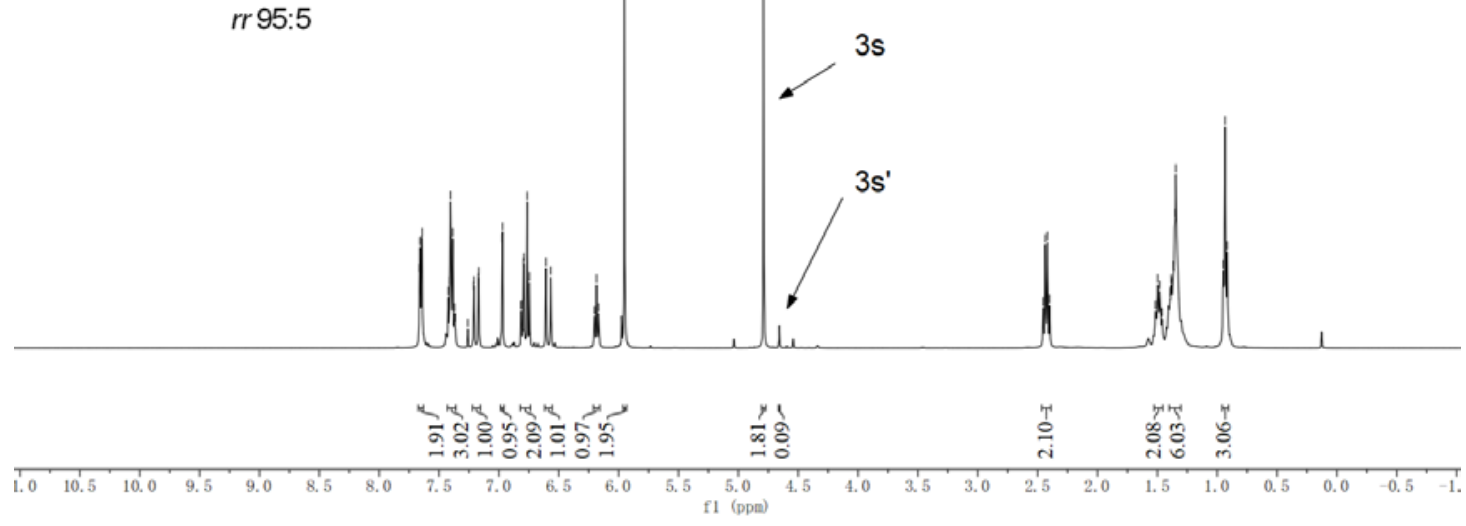

${ }^{1} \mathrm{H}$ NMR (400 MHz, $\mathrm{CDCl}_{3}$ ) spectrum of 3s 

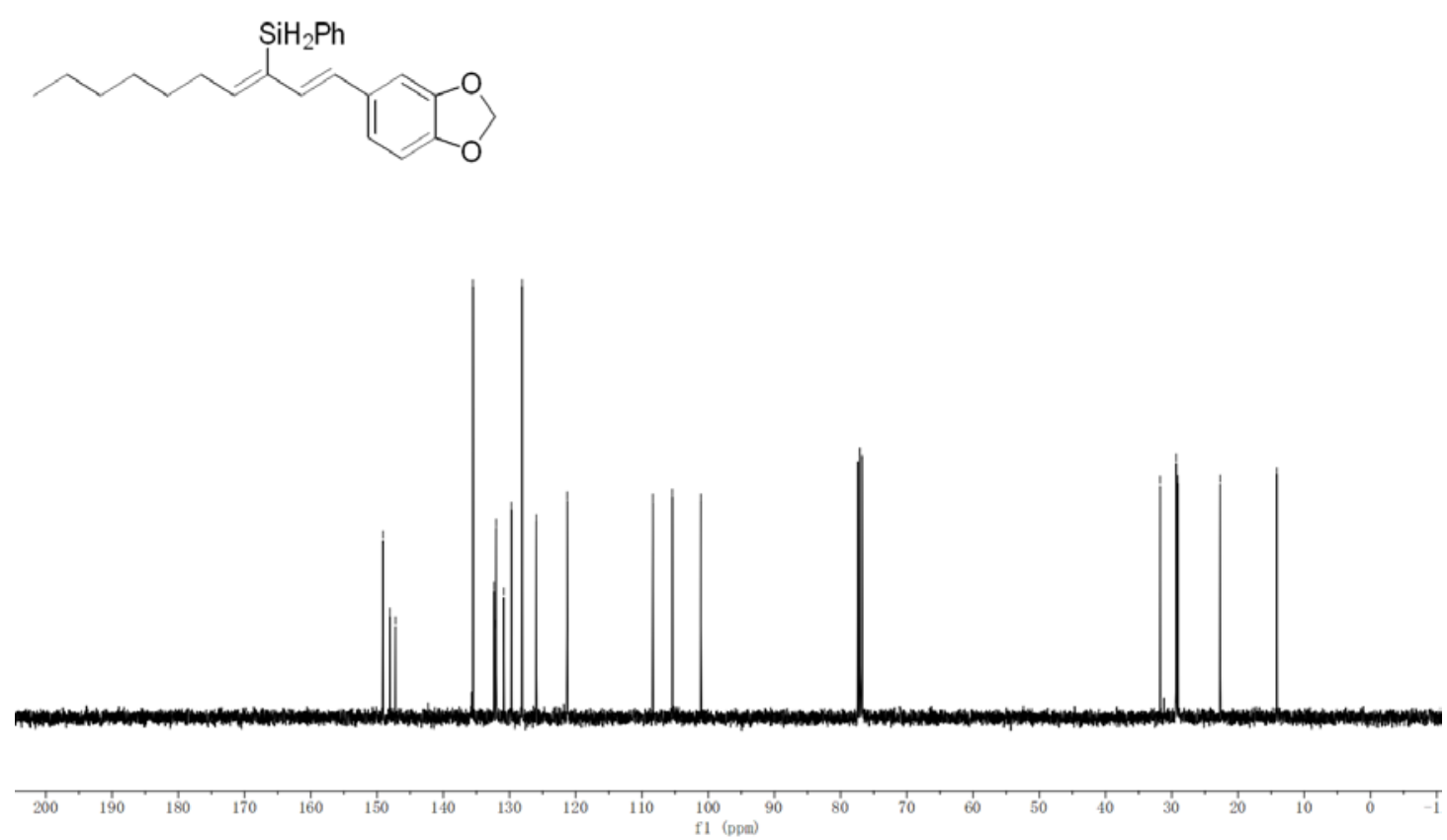

${ }^{13} \mathrm{C}$ NMR (101 MHz, $\left.\mathrm{CDCl}_{3}\right)$ spectrum of 3s

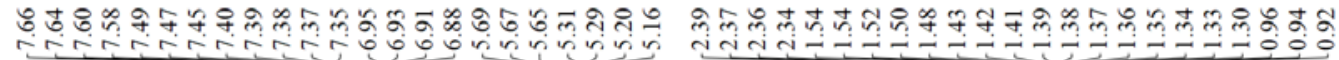<smiles>C#CCCCCC</smiles>

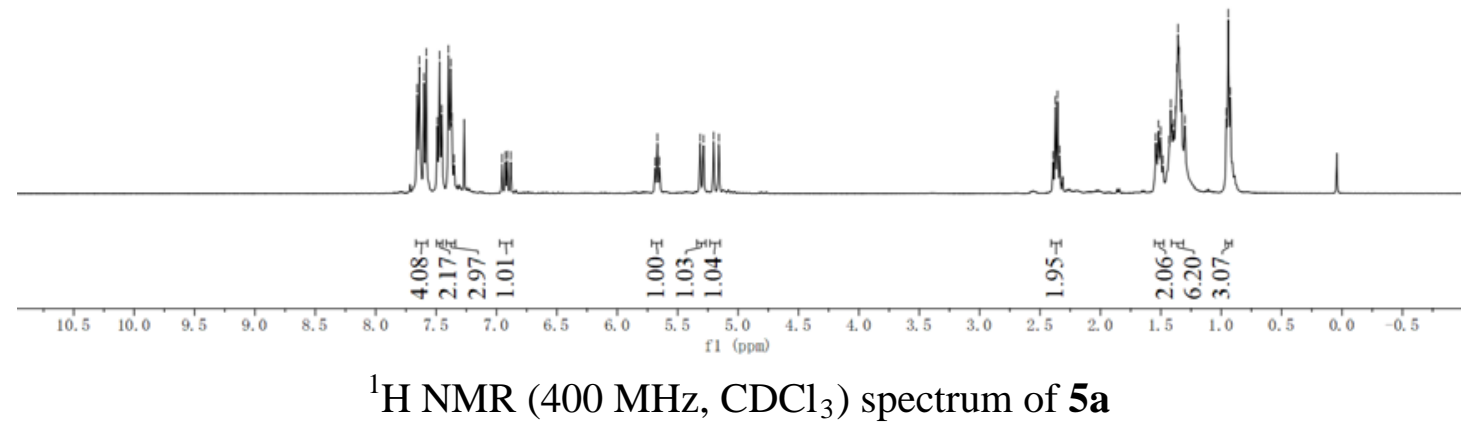



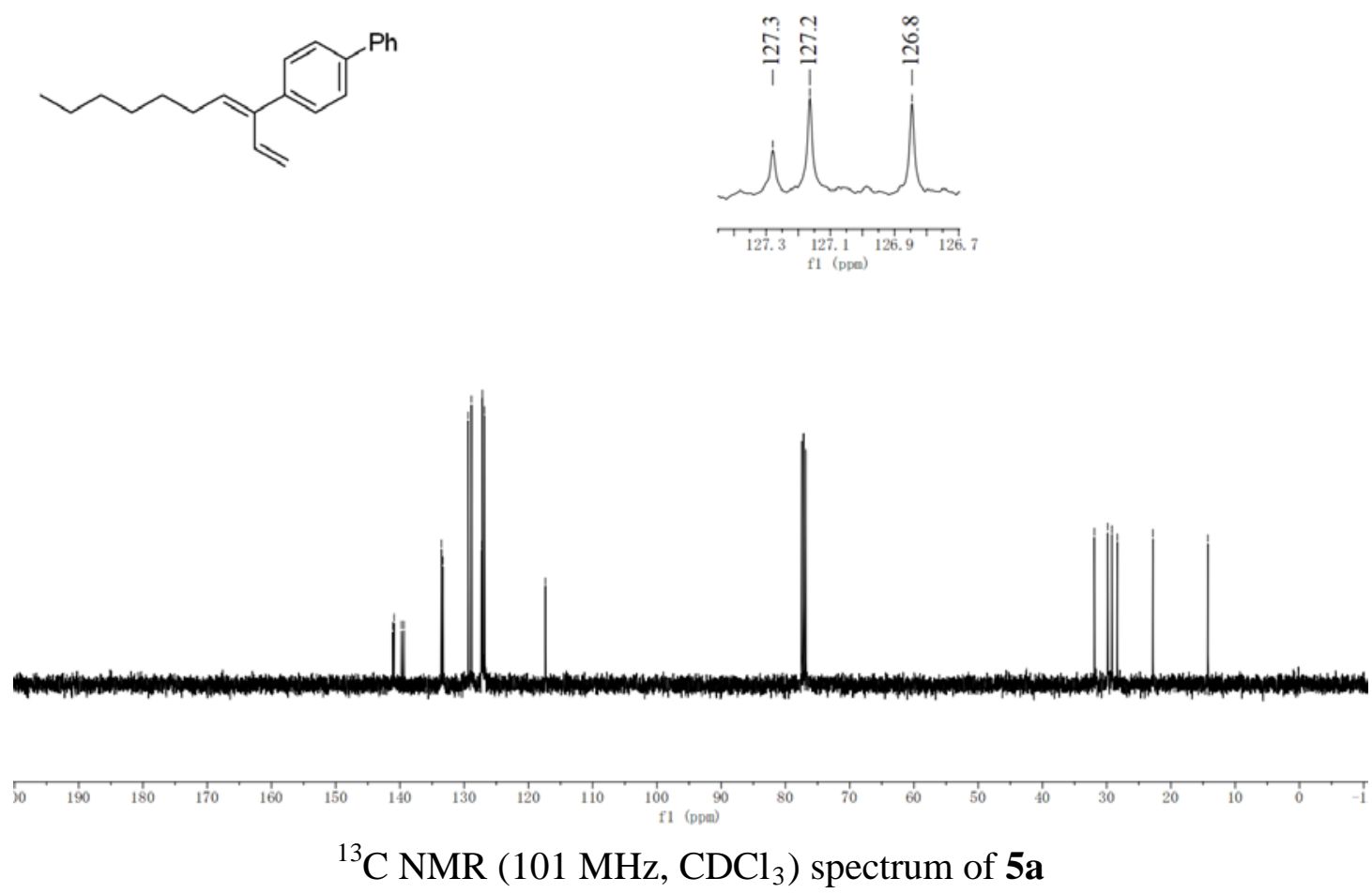

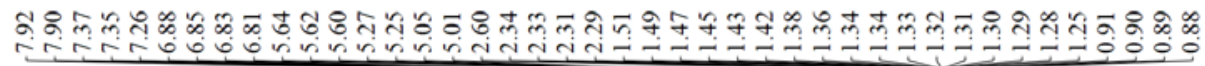
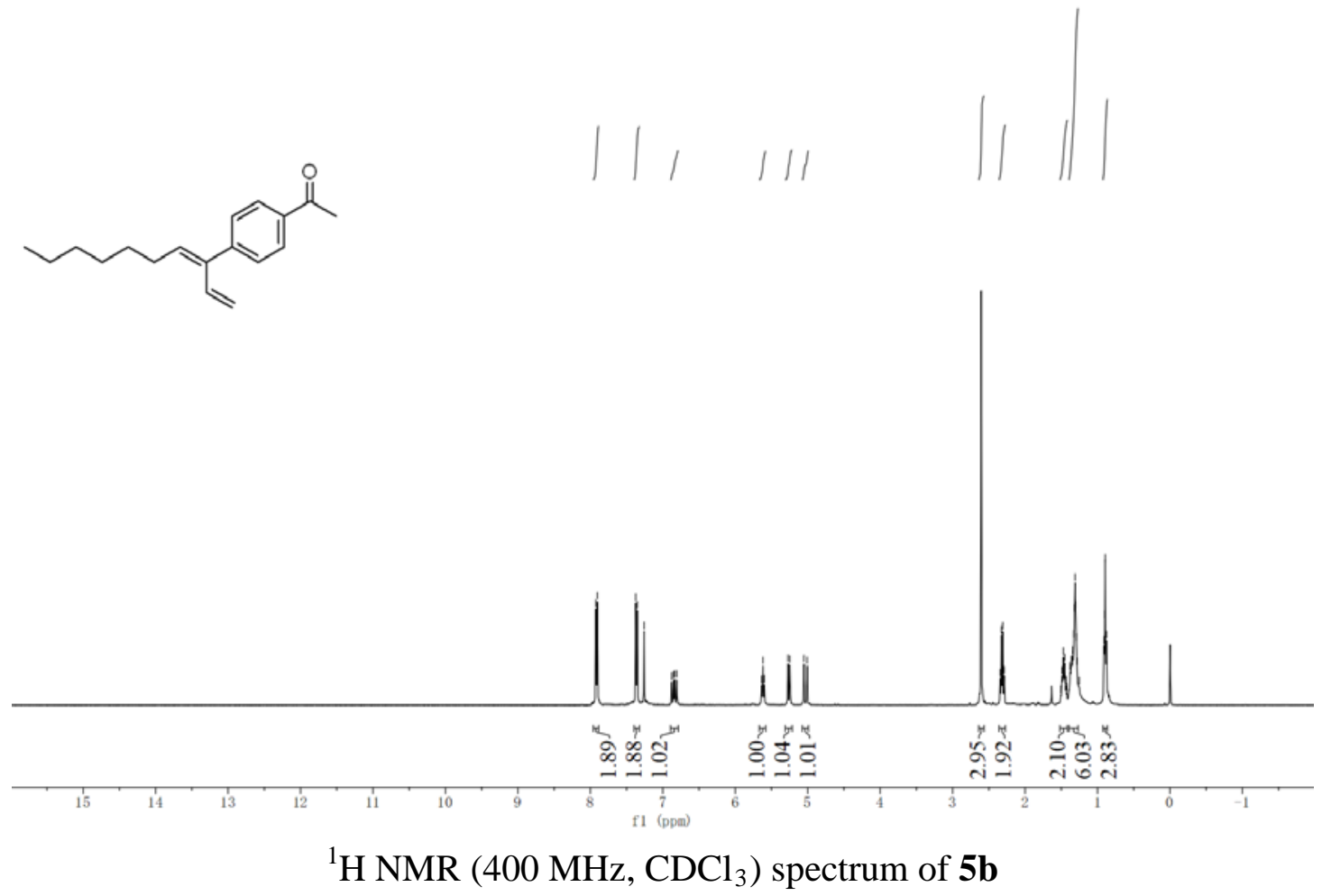


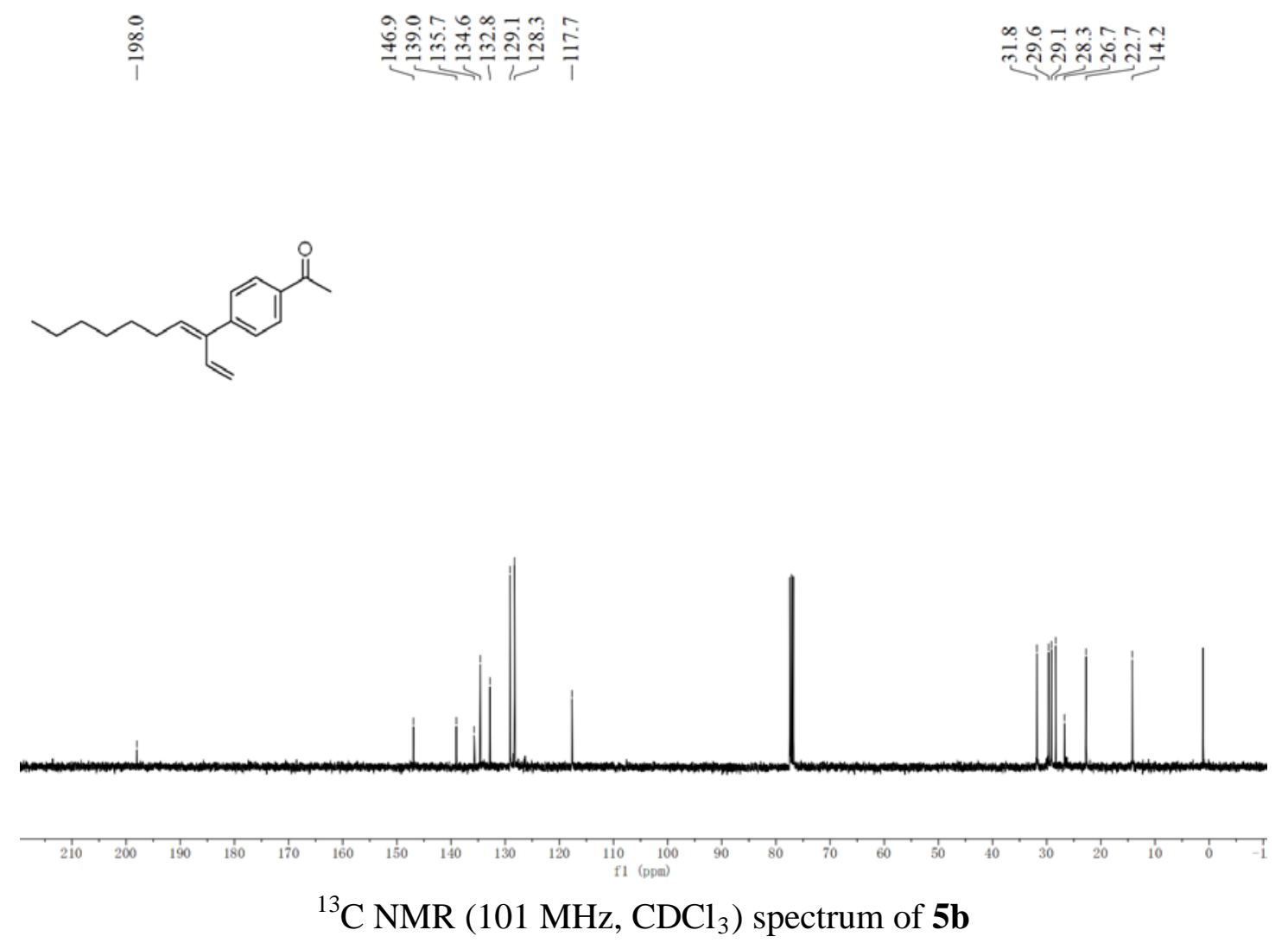

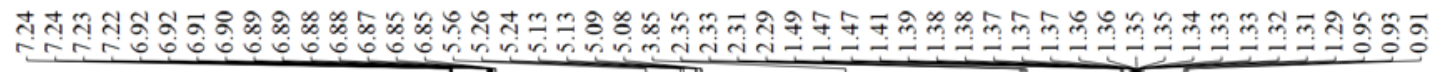
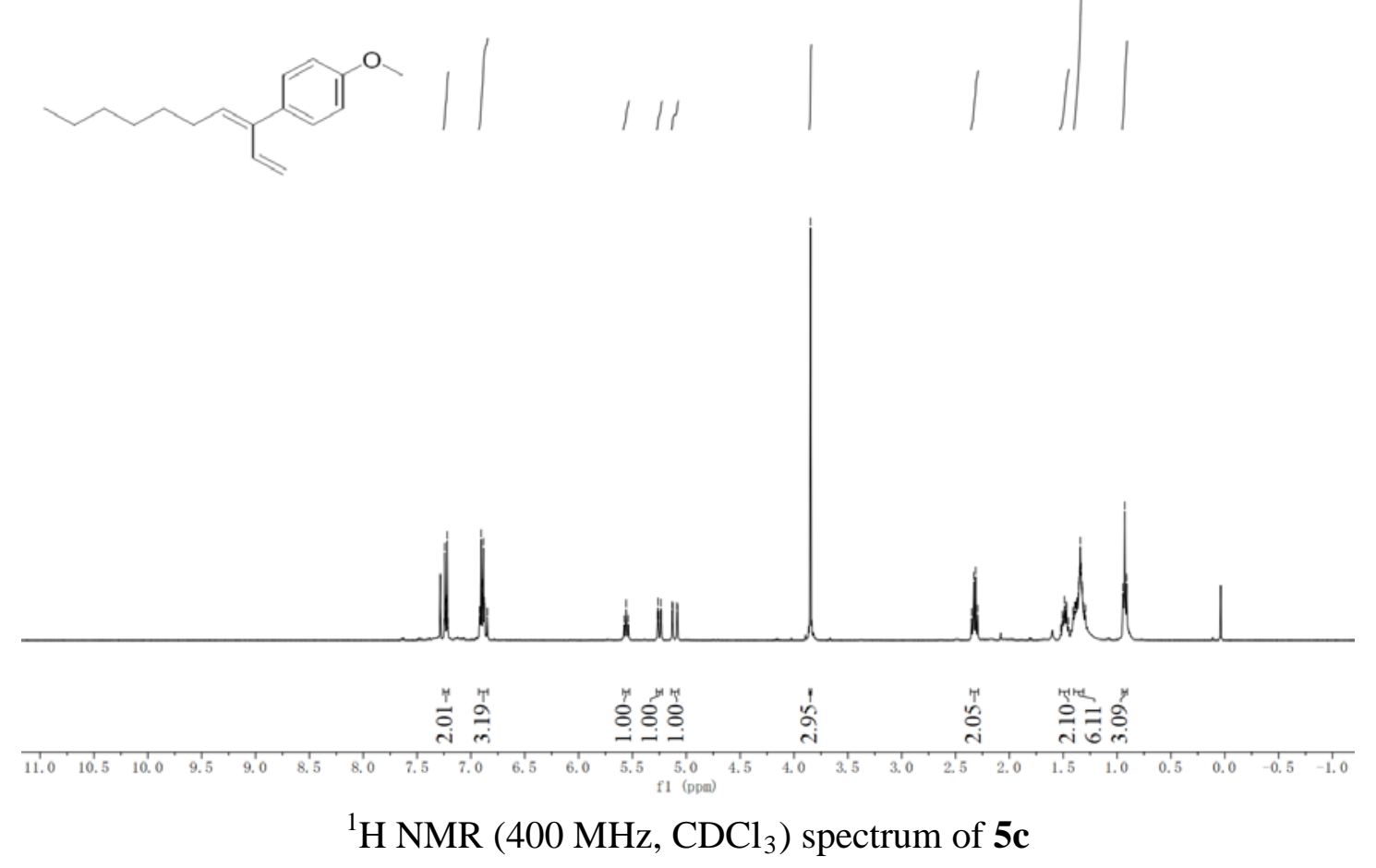


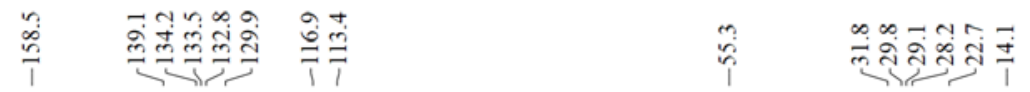
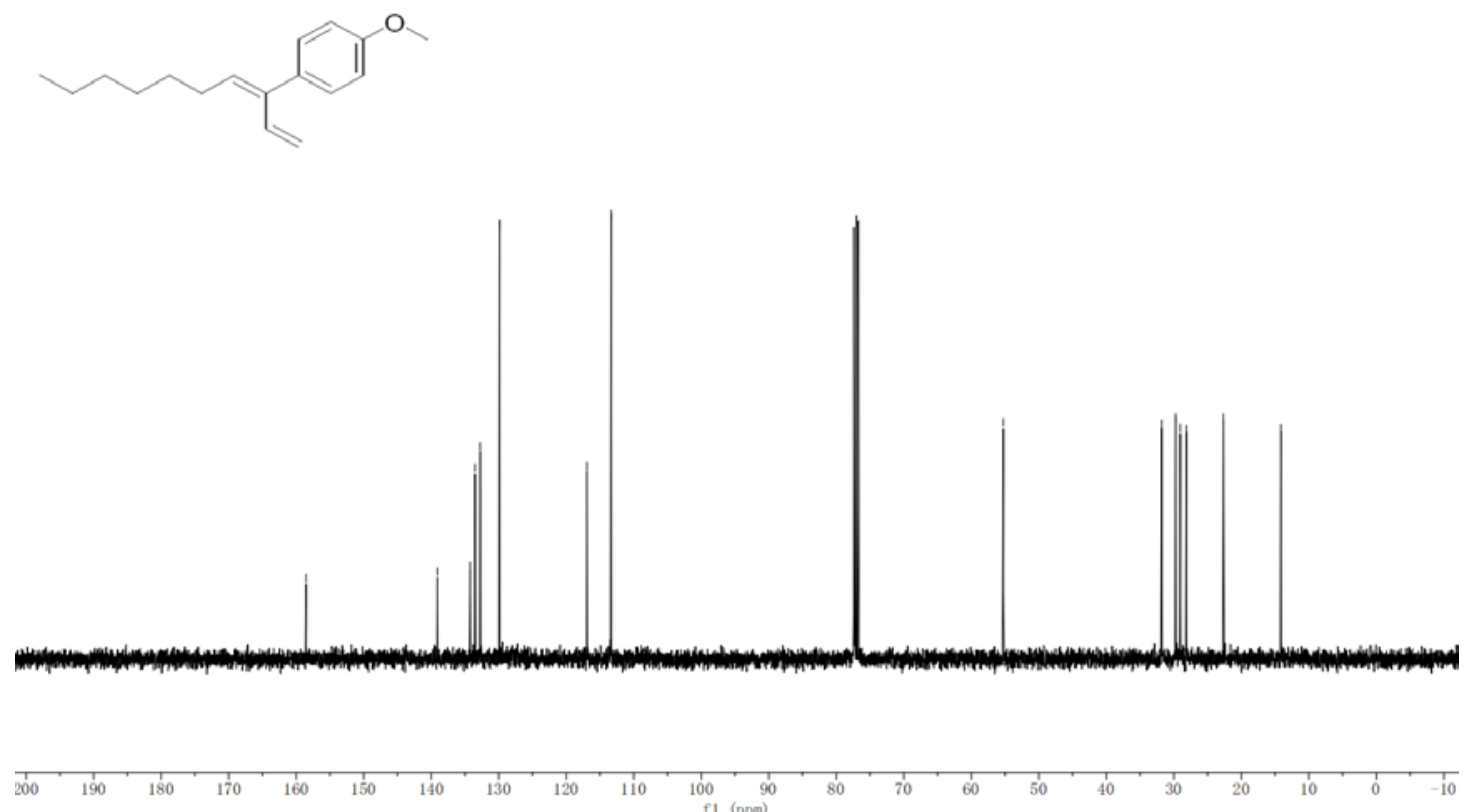

${ }^{13} \mathrm{C}$ NMR $\left(101 \mathrm{MHz}, \mathrm{CDCl}_{3}\right)$ spectrum of 5c 


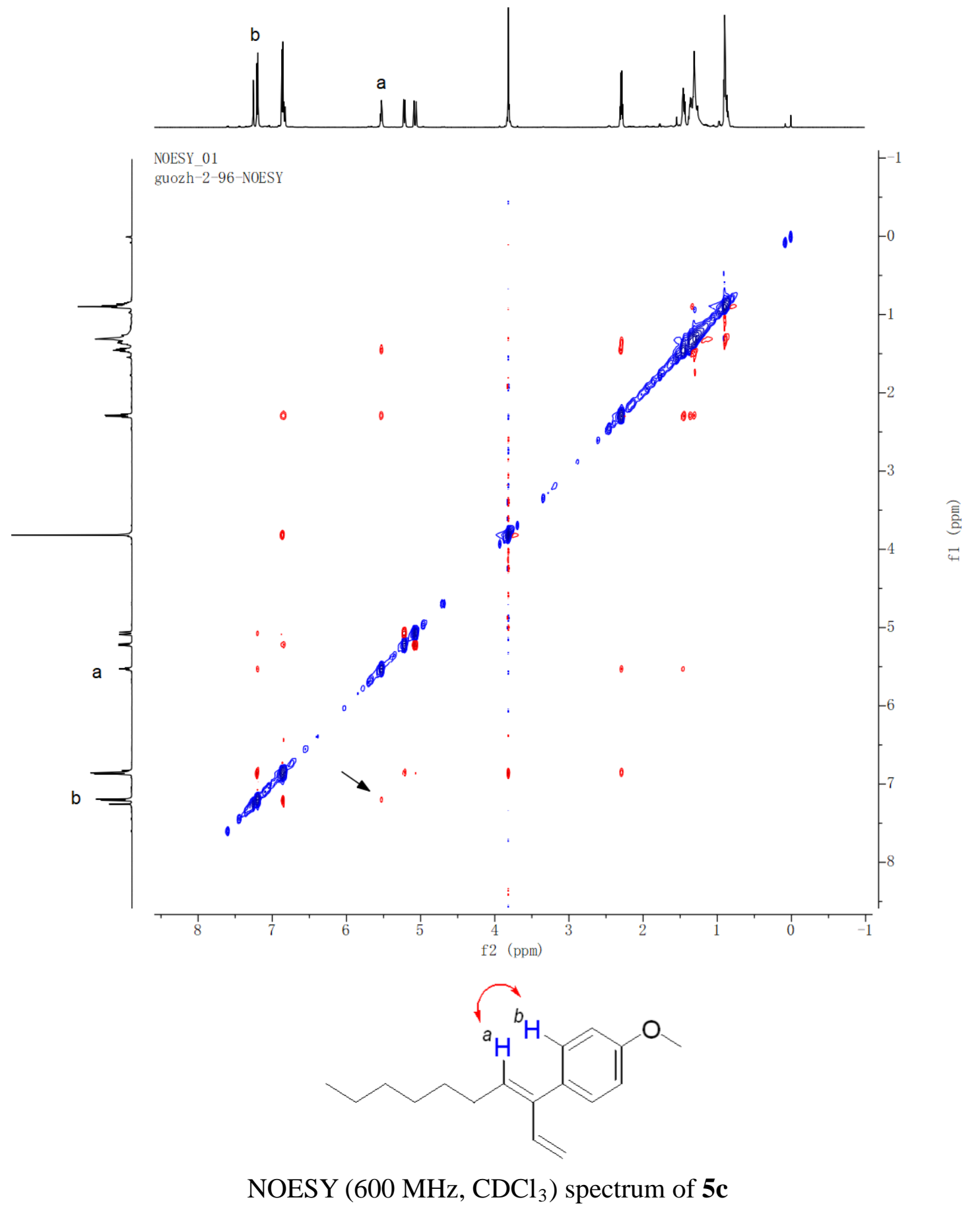



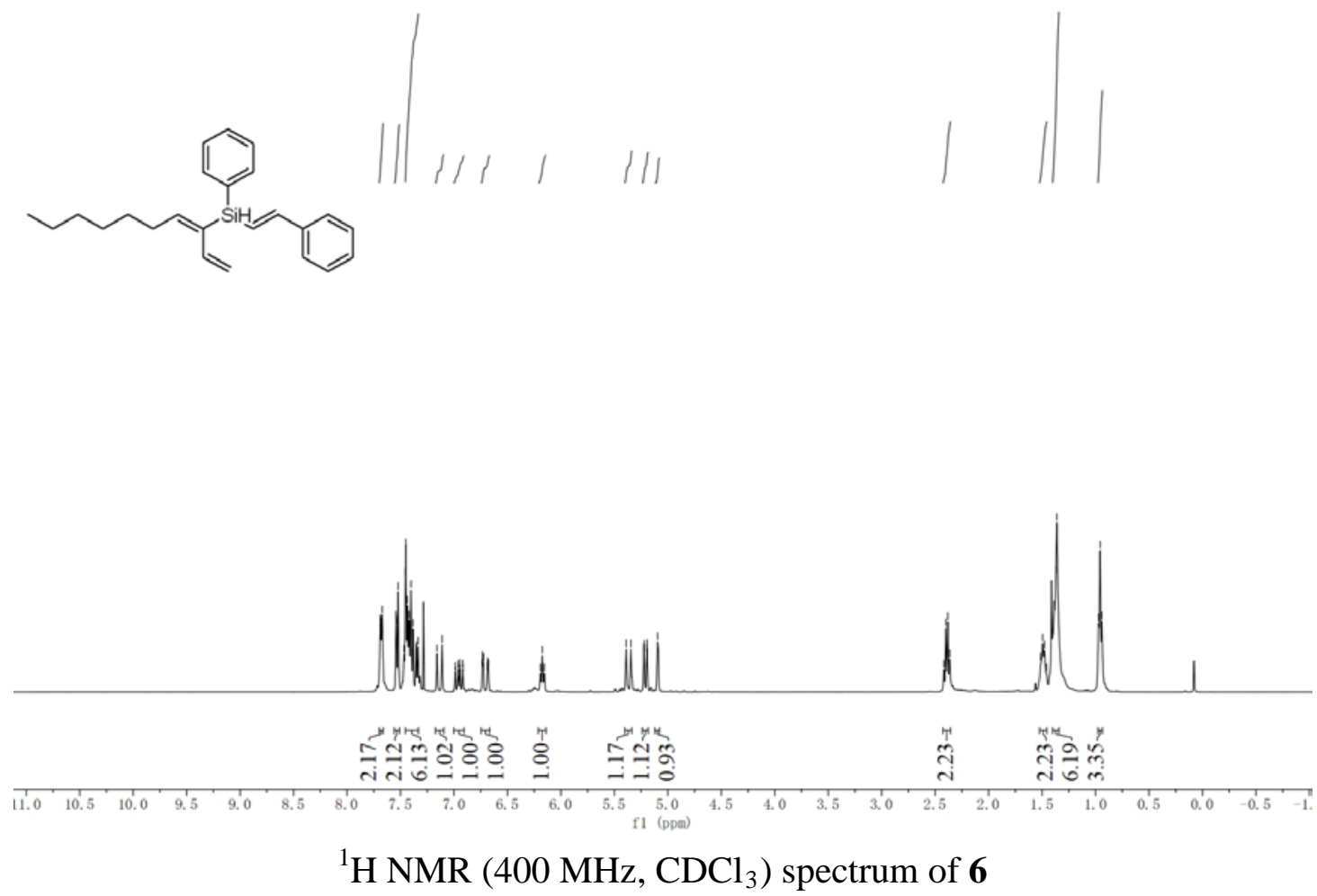

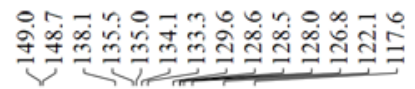

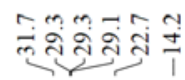
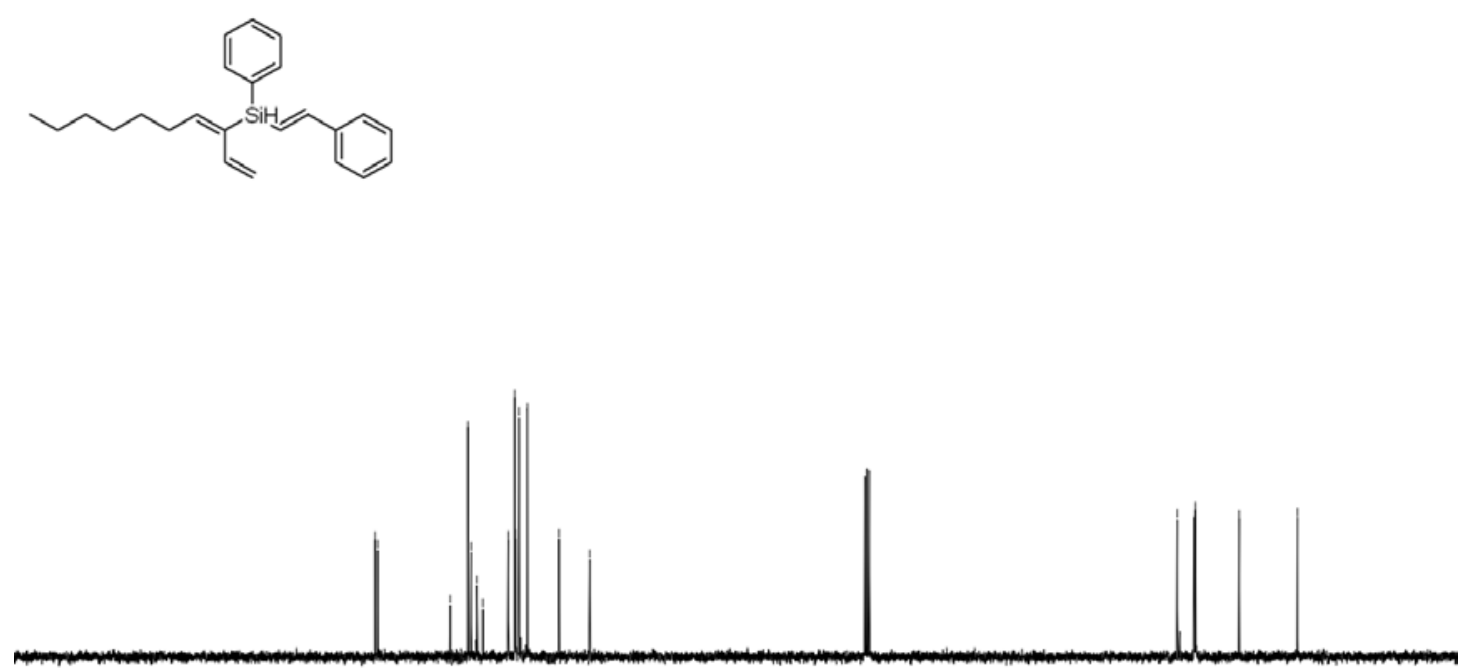

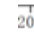
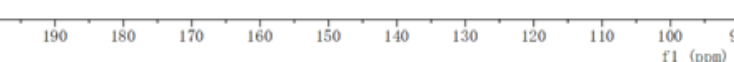

${ }^{3} \mathrm{C}$ NMR (101 MHz, $\mathrm{CDCl}_{3}$ ) spectrum of 6 


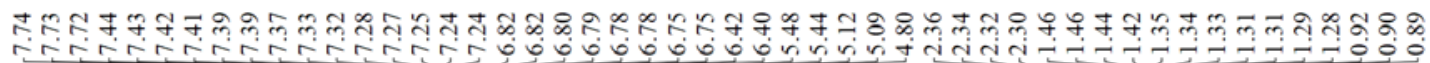

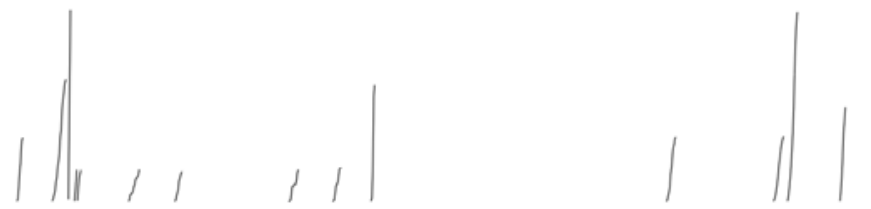

$\gamma^{\mathrm{Si}(\mathrm{OBn})_{2} \mathrm{Ph}}$

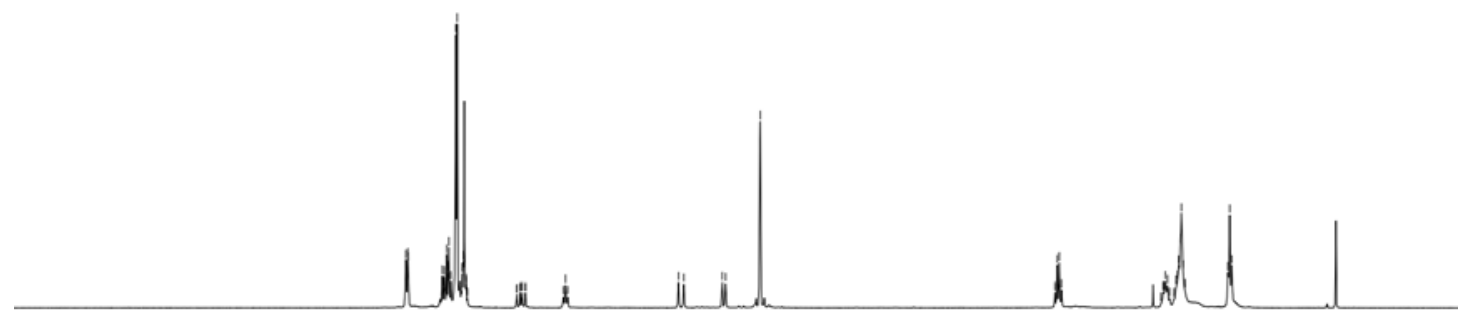

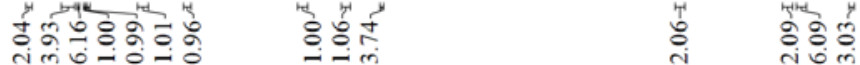

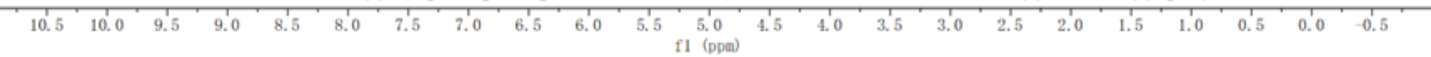

${ }^{1} \mathrm{H}$ NMR (400 MHz, $\mathrm{CDCl}_{3}$ ) spectrum of 7

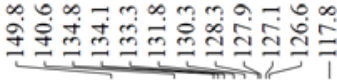
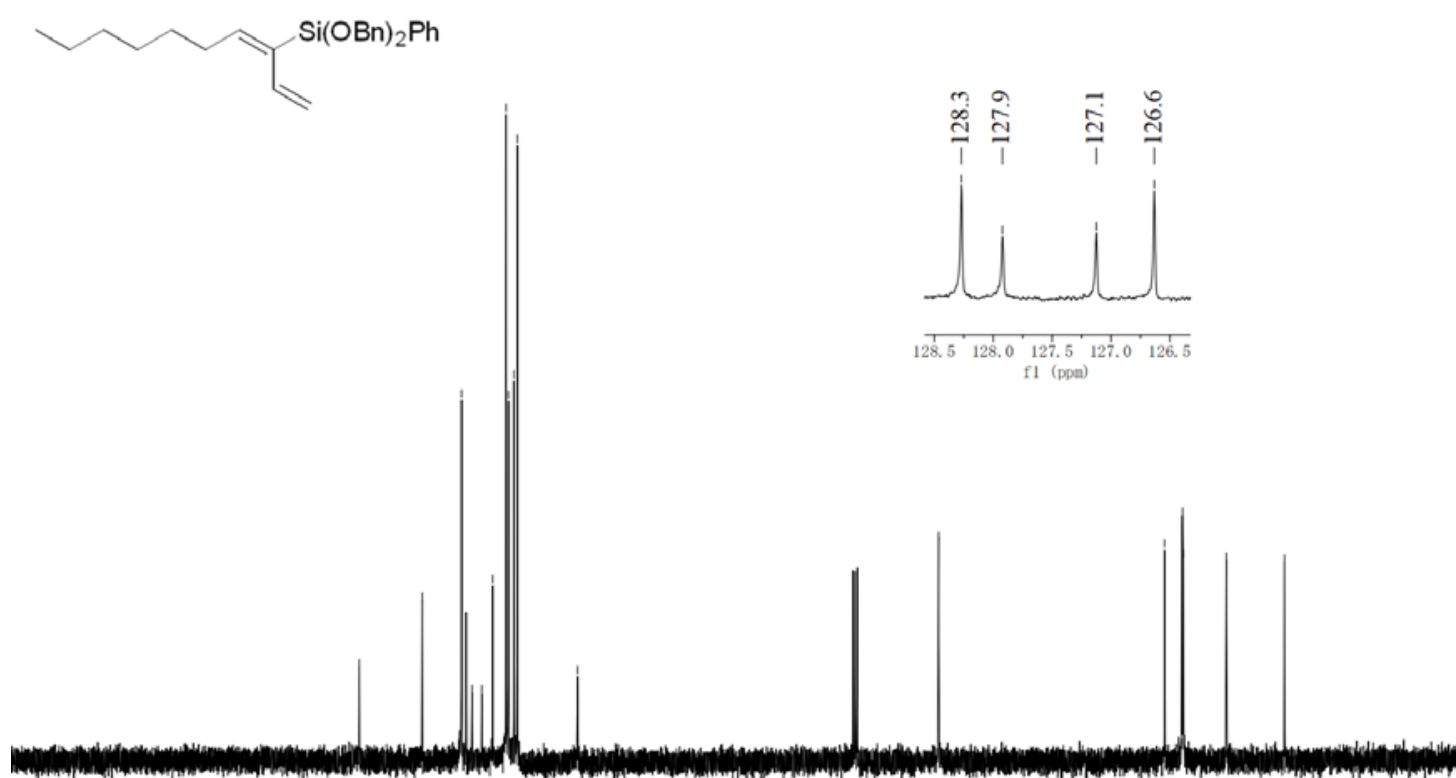

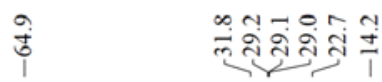

$\begin{array}{llllllll}128.5 & 128.0 & 127.5 & 127.0 & 126.5\end{array}$

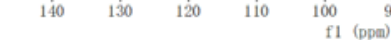

${ }^{13} \mathrm{C}$ NMR (101 MHz, $\mathrm{CDCl}_{3}$ ) spectrum of 7 

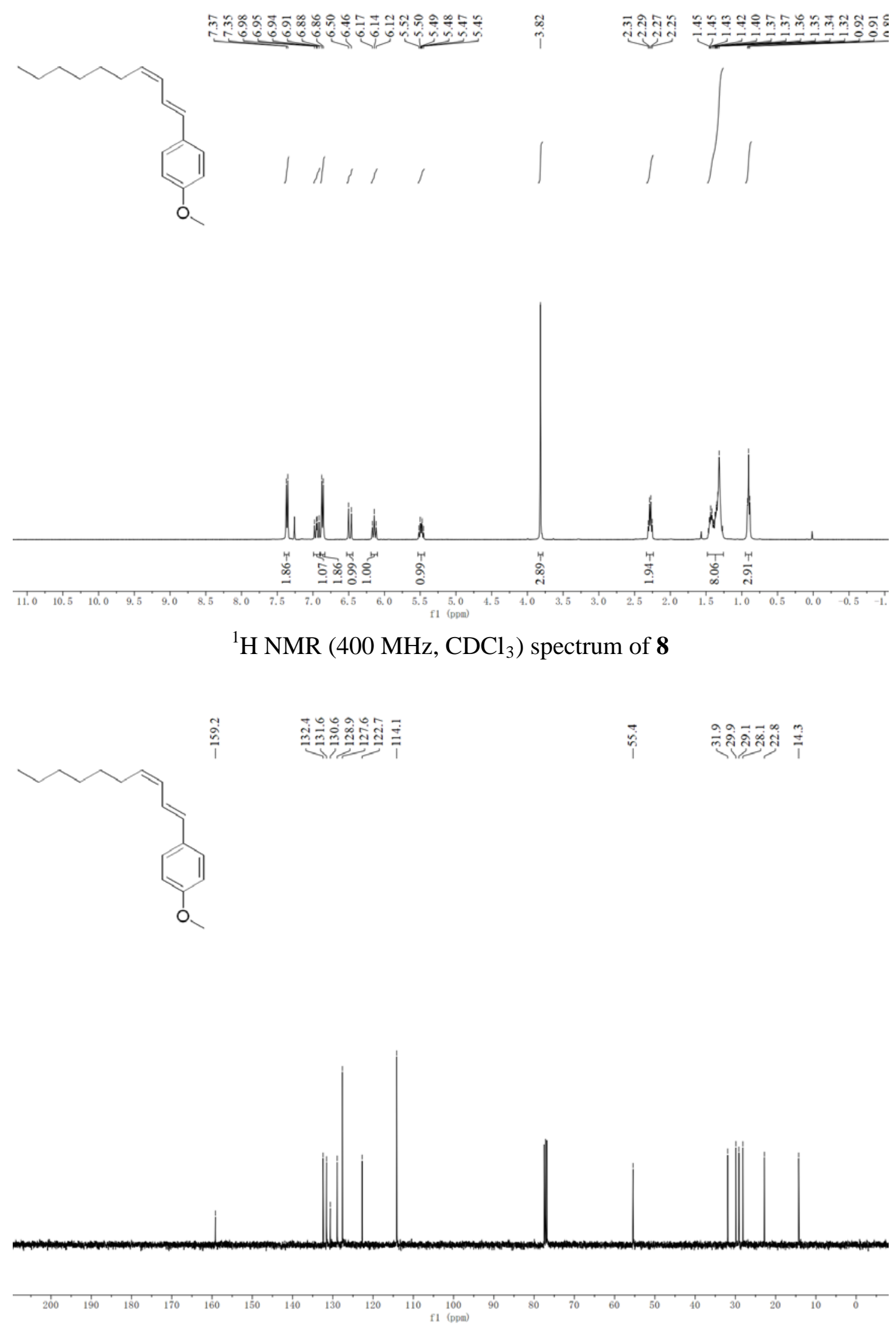

${ }^{13} \mathrm{C}$ NMR (101 MHz, $\mathrm{CDCl}_{3}$ ) spectrum of 8 